MPP-2011-136

\title{
Six-dimensional $(1,0)$ effective action of F-theory via M-theory on Calabi-Yau threefolds
}

\author{
Federico Bonetti and Thomas W. Grimm 1 \\ Max-Planck-Institut für Physik, \\ Föhringer Ring 6, 80805 Munich, Germany
}

\begin{abstract}
The six-dimensional effective action of F-theory compactified on a singular elliptically fibred Calabi-Yau threefold is determined by using an M-theory lift. The low-energy data are derived by comparing a circle reduction of a general six-dimensional $(1,0)$ gauged supergravity theory with the effective action of M-theory on the resolved Calabi-Yau threefold. The derivation includes six-dimensional tensor multiplets for which the (anti-) self-duality constraints are imposed on the level of the five-dimensional action. The vector sector of the reduced theory is encoded by a non-standard potential due to the GreenSchwarz term in six dimensions. This Green-Schwarz term also contains higher curvature couplings which are considered to establish the full map between anomaly coefficients and geometry. F-/M-theory duality is exploited by moving to the five-dimensional Coulomb branch after circle reduction and integrating out massive vector multiplets and matter hypermultiplets. The associated fermions then generate additional Chern-Simons couplings at one-loop. Further couplings involving the graviphoton are induced by quantum corrections due to excited Kaluza-Klein modes. On the M-theory side integrating out massive fields corresponds to resolving the singularities of the Calabi-Yau threefold, and yields intriguing relations between six-dimensional anomalies and classical topology.
\end{abstract}

December, 2011

\footnotetext{
${ }^{1}$ bonetti, grimm at mppmu.mpg.de
} 


\section{Contents}

1 Introduction $\quad 2$

2 F-theory in six dimensions

2.1 F-theory via M-theory . . . . . . . . . . . . . . 4

2.2 Elliptically fibred Calabi-Yau threefolds . . . . . . . . . . . . . . 6

3 Circle compactification from six to five dimensions 9

3.1 Generalities on $6 \mathrm{~d}(1,0)$ supergravity . . . . . . . . . . . . . . . 10

3.2 Kaluza-Klein reduction on the circle . . . . . . . . . . . . . . . 14

3.3 Moving to the Coulomb branch . . . . . . . . . . . . . 17

3.4 The 5 d effective action and its canonical form . . . . . . . . . . . 18

3.5 Higher order curvature corrections . . . . . . . . . . . . . . . . 23

4 M-theory on a Calabi-Yau threefold 24

4.1 M-theory action on an elliptic Calabi-Yau threefold . . . . . . . . . 25

4.2 Higher order curvature corrections . . . . . . . . . . . . . . 28

5 F-theory lift and one-loop corrections

5.1 Classical action in the F-theory lift . . . . . . . . . . . . . . . 30

5.2 Completing the duality using one-loop corrections . . . . . . . . . . . 33

5.3 Origin of the one-loop Chern-Simons couplings . . . . . . . . . . 36

6 Conclusions $\quad 40$

A Notations and conventions $\quad 42$

B Anomalies in 6d supergravity

C Two-derivative $6 \mathrm{~d}(1,0)$ supergravity on a circle 44

D Calabi-Yau reference formulae 4

E 11d supergravity on a Calabi-Yau threefold 49 


\section{Introduction}

The study of effective theories arising in string compactifications is of crucial importance both from a conceptional as well as phenomenological point of view. It is now believed that there is a vast landscape of four-dimensional effective theories with minimal or no supersymmetry arising in string theory, but it is an open problem to systematically characterize these theories [1, 2]. A systematic study becomes more tractable in compactifications to higher dimensions and with more supersymmetry. Highly supersymmetric compactifications have a more constrained effective theory, and arise from restricted classes of candidate string constructions. In the maximally supersymmetric case the theory and compactification geometry are in fact almost unique.

An intermediate scenario is provided by six-dimensional $(6 \mathrm{~d})(1,0)$ supergravity theories [3]. While there are constraints both from supersymmetry and anomalies in this dimension, the moduli space of these theories still permits a rich structure and is not fixed by the symmetries. The $(1,0)$ multiplets in the spectrum are the gravity multiplet, a number of tensor and vector multiplets, as well as neutral and matter hypermultiplets. A special complication arises from the fact that in six dimensions the $(1,0)$ two-form tensors in the tensor multiplets and the gravity multiplet obey duality constraints. The two-form in the gravity multiplet has a self-dual field strength, while the two-forms in the tensor multiplets will admit an anti-self-dual field strength. This fact makes it hard to give a Lagrangian formulation for the dynamics of these forms. While such formulations exist [4], we will take a different route in this work. Our $6 \mathrm{~d}$ actions will be formulated as pseudo-actions which yield equations of motions for the tensor fields which still need to be additionally restricted by imposing the self- and anti-self-duality constraints [5, 6, 7, 8]. Moreover, our computations will proceed by first determining a five-dimensional (5d) action for which these conditions can be consistently imposed on the level of the action. We will see that this transdimensional treatment is natural in connecting compactifications of F-theory and M-theory. Recently, a transdimensional treatment was suggested to study the M5-brane action with self-dual non-Abelian two-forms [9, 10, 11].

In the last years a systematic study of six-dimensional $(1,0)$ supergravity theories has been undertaken to study the consistency conditions imposed by quantum gravity [3]. In $6 \mathrm{~d}$ there are gravitational, gauge as well as mixed anomalies. These impose constraints on the number of multiplets, and link the matter spectrum to the anomaly coefficients; see e.g. [12, 13, 14]. A fruitful starting point has been to ask for a realization of these supergravity theories as a compactification of F-theory on Calabi-Yau threefolds [15, 16, 17, 18, 19, 20, 21]. These threefolds have to be elliptically fibred with a base space being a Kähler twofold. At the loci in the base where the elliptic fibre becomes singular, the dilaton-axion, parameterizing the complex structure of the elliptic fibre, indicates the existence of seven-brane sources. These seven-branes wrap complex curves in the base. Two seven-branes can intersect at points at which strings ending on different branes yield new massless matter hypermultiplets in the effective theory. This gives the embedding of six-dimensional gauge theories with matter in a general geometric framework. Note 
that in order to obtain non-Abelian gauge groups the elliptic Calabi-Yau threefold $Y_{3}$ has to be singular itself. For Calabi-Yau singularities localized along a single seven-brane divisor one can infer the gauge group $G$ at co-dimension one in the base. The singularity enhancements at co-dimension two in the base predict the representations of matter fields [22, 23, 24].

To study the $6 \mathrm{~d}(1,0)$ effective action arising by compactifications of F-theory on an elliptically fibred Calabi-Yau threefold, we take a detour via M-theory. Our analysis will be analogous to the $4 \mathrm{~d} / 3 \mathrm{~d}$ treatment of F-theory on Calabi-Yau fourfolds presented in [25, 26], but will be more refined and use the enhanced constraints of $6 \mathrm{~d}(1,0)$ supersymmetry and anomalies. M-theory and F-theory on the same Calabi-Yau manifold are connected by a certain limit which shrinks the elliptic fibre in M-theory and grows an extra dimension required to the match with F-theory. The simplest physical description of this limit is provided by considering M-theory on a two-torus. Shrinking the size of the torus, one ends up in a Type IIA string set-up on a small circle. Performing a T-duality along this circle leads to a Type IIB string compactifications on a large circle. Indeed, if the torus shrinks to zero size, the Type IIB set-up grows an extra dimension. One can extend this limit adiabatically to elliptic fibrations. Furthermore, also branes and flux sources can be traced through this duality. We recall more details on this duality and the geometry of elliptically fibred Calabi-Yau threefolds in section 2 .

Since we want to determine the characteristic data of the $6 \mathrm{~d}$ F-theory effective action, we start with a rather general $6 \mathrm{~d}(1,0)$ pseudo-action with a non-Abelian gauge group $G$, and a generalized Green-Schwarz term to cancel 6d anomalies [5, 6, 7]. The selfduality of the tensors is imposed on the level of the equations of motion. We perform the Kaluza-Klein reduction on a circle, and derive an actual $5 \mathrm{~d}$ effective action for the Kaluza-Klein zero-modes in section 3. We show how the self-duality can now be imposed on the action level, and determine the characteristic data of the $5 \mathrm{~d} \mathcal{N}=2$ theory. In particular, we find that the kinetic terms of the $5 \mathrm{~d}$ vectors are encoded by a real function $\mathcal{N}^{\mathrm{F}}$, which is homogeneous of degree three. It is interesting to point out that it contains a non-polynomial term which is not allowed in a standard $5 \mathrm{~d} \mathcal{N}=2$ supergravity theory. This correction is induced by the fact that the $6 \mathrm{~d}$ theory contained a classically nongauge invariant Green-Schwarz term to cancel 6d one-loop anomalies. Our findings are then interpreted as counterterms in five dimensions, following the suggestion of [17]. In order to prepare the ground for the comparison with the M-theory reduction, it will be crucial to comment on the modifications when moving to the Coulomb branch of the $5 \mathrm{~d}$ gauge theory. Furthermore, also higher curvature terms are required in $6 \mathrm{~d}$ for anomaly cancellation, and we provide a partial dimensional reduction which will be compared with the M-theory result.

To determine the $6 \mathrm{~d}$ characteristic data in terms of the geometric data of the compactification threefold, we also determine the $5 \mathrm{~d}$ M-theory effective action in section 4 . The derivation is performed on a fully resolved Calabi-Yau threefold $\tilde{Y}_{3}$. This implies that the $5 \mathrm{~d}$ gauge theory will be in the Coulomb branch, and all M2-brane states wrapped on the elliptic fibre and the resolution cycles will be massive. The resulting $5 \mathrm{~d} \mathcal{N}=2$ action 
has already been known in the literature [27]. Also higher curvature corrections have been dimensionally reduced from eleven to five dimensions [28]. It was shown in [28] that the second Chern class of the threefold $\tilde{Y}_{3}$ determines 5 d higher curvature couplings of the form $A \wedge \operatorname{tr} \mathcal{R} \wedge \mathcal{R}$, with $A$ being a 5 d vector and $\mathcal{R}$ being the 5 d curvature two-form.

In the comparison of the general $6 \mathrm{~d} / 5 \mathrm{~d}$ reduction with the M-theory reduction in section 5, we argue that the latter does not only contain the classical terms but also certain one-loop corrections. We identify the F-theory limit of M-theory which leads to a perfect match of the classical terms and allows us to extract all characteristic data for the $6 \mathrm{~d}(1,0)$ theory in terms of the geometry of the resolved Calabi-Yau threefold $\tilde{Y}_{3}$. This includes the geometric data determining the classical metrics on the $6 \mathrm{~d}$ vector, tensor and hypermultiplet moduli spaces. Including a comparison of the dimensionally reduced higher curvature terms, we also infer the discrete data determining the $6 \mathrm{~d}$ Green-Schwarz term and hence encode $6 \mathrm{~d}$ anomalies. Our results confirm more indirect arguments using the Chern-Simons action of seven-branes [18, 29]. Furthermore, our results agree with the analysis of $6 \mathrm{~d}$ anomalies presented in [19].

Remarkably, we identify several terms in the 5 d M-theory reduction on $\tilde{Y}_{3}$ which do not arise in the classical $6 \mathrm{~d} / 5 \mathrm{~d}$ reduction. We argue using [30, 31] that this is due to the fact that there are one-loop corrections in the $6 \mathrm{~d} / 5 \mathrm{~d}$ reduction which arise form two sources: (1) in going to the Coulomb branch, there are massive charged hypermultiplets, and massive vector multiplets containing the W-bosons which have to be integrated out, (2) in the dimensional reduction there are massive Kaluza-Klein modes for all $6 \mathrm{~d}$ multiplets. In particular, we argue that massive fermions running in the loop generate constant corrections to the 5d Chern-Simons terms of the from $A \wedge F \wedge F$, with $F$ being the $5 \mathrm{~d}$ gauge fields. Integrating out $5 \mathrm{~d}$ massive Kaluza-Klein modes of $6 \mathrm{~d}$ chiral fields also generates one-loop Chern-Simons couplings $A^{0} \wedge F^{0} \wedge F^{0}$ and $A^{0} \wedge \operatorname{tr}(\mathcal{R} \wedge \mathcal{R})$ for the $5 \mathrm{~d}$ vector zero-mode $A^{0}$ arising from the reduction of the $6 \mathrm{~d}$ gravity multiplet. Both coefficients depend on the number of $6 \mathrm{~d}$ tensor multiplets. The comparison with the M-theory result is expected to yield the $6 \mathrm{~d}$ anomaly conditions.

\section{F-theory in six dimensions}

This section is devoted to a brief account on the F-theory set-up, applied to the construction of $6 \mathrm{~d}$ models. Firstly, we recall the basics of F-/M-theory duality and make some comments about the Type IIB picture of F-theory vacua. Secondly, we develop a minimal mathematical toolkit to describe elliptically fibred Calabi-Yau threefolds.

\section{$2.1 \quad$ F-theory via M-theory}

F-theory [15] is a twelve-dimensional geometric framework introduced to capture some crucial non-perturbative aspects of Type IIB vacua in presence of seven-branes. One of the most efficient ways to extract information about F-theory vacua is given by dual- 
ity with M-theory compactifications [2]. Since we will follow this strategy throughout the paper, let us briefly review some basic material about F-/M-theory duality and its application to the study of $6 \mathrm{~d}$ vacua.

Consider M-theory on the product manifold

$$
\mathcal{M}_{11}=T^{2} \times B_{2} \times \mathbb{R}^{1,4}
$$

where $T^{2}$ is a two-torus, $B_{2}$ is a Kähler manifold of complex dimension two, and $\mathbb{R}^{1,4}$ is $5 \mathrm{~d}$ Minkowski spacetime. The metric on the torus can be written as

$$
d s_{T^{2}}^{2}=\frac{v^{0}}{\operatorname{Im} \tau}\left[\left(d x_{A}+\operatorname{Re} \tau d x_{B}\right)^{2}+(\operatorname{Im} \tau)^{2} d x_{B}^{2}\right] .
$$

Here $x_{A}, x_{B}$ are real coordinates with period $1, \tau$ is the complex structure parameter, and $v^{0}$ is the volume of the torus. The canonical coordinates $x_{A}, x_{B}$ parameterize the two one-cycles which we name $A$ - and $B$-cycle, respectively. Upon compactification along a small $A$-cycle, M-theory reduces to Type IIA string theory. An application of T-duality acting on the $B$-cycle results in Type IIB string theory on the background

$$
\mathcal{M}_{10}=S^{1} \times B_{2} \times \mathbb{R}^{1,4}
$$

where the circle $S^{1}$ corresponds to the $B$-cycle. Note that in this duality the complex structure parameter $\tau$ in (2.2) is identified with dilaton-axion $\tau=C_{0}+i e^{-\phi}$, where $C_{0}$ is the RR scalar and $\phi$ is the dilaton. In this way, $S L(2, \mathbb{Z})$ modular invariance of Type IIB is interpreted as the $S L(2, \mathbb{Z})$ reparameterization symmetry of the complex structure parameter of $T^{2}$. Furthermore, in the limit of vanishing $v^{0}$, the size of the compact $S^{1}$ becomes infinite, thus leading effectively to Type IIB on

$$
\mathcal{M}_{10}^{\prime}=B_{2} \times \mathbb{R}^{1,5}
$$

where $\mathbb{R}^{1,5}$ denotes $6 \mathrm{~d}$ Minkowski spacetime. The present discussion can be generalized to the case in which $\mathcal{M}_{11}$ is a $T^{2}$-fibration over $B_{2} \times \mathbb{R}^{1,4}$, repeating the argument fibrewise. We require $T^{2}$ to depend holomorphically on the complex coordinates of $B_{2}$. More precisely, the 11d background can be written as

$$
\mathcal{M}_{11}=Y_{3} \times \mathbb{R}^{1,5}
$$

where $Y_{3}$ is an elliptic fibration with zero-section over the base $B_{2}$ with fibres being possibly singular elliptic curves. In order to preserve a fraction of supersymmetry, $Y_{3}$ must be a Calabi-Yau manifold. In summary we are thus led to consider elliptically fibred Calabi-Yau threefolds. We introduce some basic facts about their geometry in subsection 2.2.

Carrying out the duality program outlined above, we end up with a Type IIB vacuum with non-trivial dilaton-axion profile $\tau$ varying along $B_{2}$. As a consequence general Ftheory vacua do not admit a perturbative description in terms of of fundamental strings and D-branes. The fundamental objects of F-theory are $(p, q)$-strings and $(p, q)$-branes, 
which are $S L(2, \mathbb{Z})$-generalizations of the fundamental strings and D-branes. A particular role is played by $(p, q)$ seven-branes which magnetically couple to $\tau$. This allows to treat them geometrically. In fact, space-time filling seven-branes are located at co-dimension one loci in $B_{2}$ at which the elliptic fibre becomes singular. More precisely, a $(p, q)$ sevenbrane can be found at a point where the $(p A+q B)$-cycle collapses. In the following we will include stacks of such seven-branes which admit a non-Abelian gauge-theory on their worldvolume.

Despite the non-perturbative nature of general F-theory vacua, the connection with M-theory will allow us to restrict to a low-energy supergravity framework. More precisely, we will compute a $6 \mathrm{~d}$ effective action for F-theory vacua through the following steps:

1. computation of the $5 \mathrm{~d} \mathcal{N}=2$ action resulting from Kaluza-Klein reduction on a circle of a general $6 \mathrm{~d}(1,0)$ supergravity action; move to the $5 \mathrm{~d}$ Coulomb branch of the gauge theory;

2. computation of the $5 \mathrm{~d} \mathcal{N}=2$ effective action of M-theory on a resolved elliptically fibred Calabi-Yau threefold;

3. comparison between the results and determination of the characteristic data which specify the $6 \mathrm{~d}(1,0)$ effective action.

In carrying out this program we will restrict to the zero-modes in both the $6 \mathrm{~d} / 5 \mathrm{~d}$ reduction as well as in the M-theory reduction. In the $6 \mathrm{~d} / 5 \mathrm{~d}$-reduction the Kaluza-Klein modes will become light in the decompactification limit and restore the dependence of the supergravity fields on all $6 \mathrm{~d}$ coordinates. Moreover, in the M-theory reduction additional M2-brane modes become relevant in the F-theory limit due to the vanishing size of the $T^{2}$-fibre. Both contributions will be neglected when working with $5 \mathrm{~d}$ massless modes only. However, the duality outlined above suggests that the massive $5 \mathrm{~d}$ corrections of both sides can also be matched. The crucial observation which we will use in our work, is that the functional dependence of the characteristic data of the supersymmetric actions on the fields should be already captured by the zero-modes. This allows us to carry out the above program and indeed determine the $6 \mathrm{~d}$ effective action of an F-theory compactification on a Calabi-Yau threefold. In addition, we find that also certain one-loop corrections can be matched under this duality and are crucial to complete the picture.

\subsection{Elliptically fibred Calabi-Yau threefolds}

As explained in the previous subsection, we want to consider elliptically fibred CalabiYau threefolds. To this end, it is useful to recall the Weierstrass description of a $T^{2}$ as a complex curve inside the weighted projective space $\mathbb{P}_{2,3,1}$, as discussed e.g. in [16, 2]. In this ambient space the $T^{2}$ is given by the equation

$$
y^{2}=x^{3}+f x z^{4}+g z^{6},
$$


modulo the identification $(x, y, z) \equiv\left(\mu^{2} x, \mu^{3} y, \mu z\right)$ for all $\mu \in \mathbb{C} \backslash\{0\}$. If $f, g$ are complex constants we are describing a specific elliptic curve. In order to describe an elliptic fibration over $B_{2}$, we have to promote $f, g$ to sections of the line bundles $-4 K,-6 K$ respectively, where $K$ denotes the canonical line bundle on $B_{2}$. Locally $f, g$ can be given as polynomials in some holomorphic coordinates of $B_{2}$.

In order to describe seven-branes we have to find the loci where the elliptic fibre degenerates. This happens at points on $B_{2}$ where the discriminant

$$
\Delta=4 f^{3}+27 g^{2}
$$

vanishes. Let us denote by $[\Delta]$ the two-form cohomology class Poincaré dual in $B_{2}$ to the divisor given by $\Delta=0$. It has been shown by Kodaira that this class $[\Delta]$ must be related to the canonical class $[K]=-c_{1}\left(B_{2}\right)$ of the base $B_{2}$ via

$$
-12[K]=[\Delta],
$$

in order for the total space $Y_{3}$ to be a Calabi-Yau manifold. Generally speaking, a singularity in the fibration may or may not yield a singularity of the whole Calabi-Yau threefold. We are thus led to represent $[\Delta]$ as

$$
[\Delta]=\sum_{A} \nu_{A}\left[S_{A}\right]+\left[\Delta^{\prime}\right]
$$

where $\left[S_{A}\right]$ are the Poincaré dual two-form classes of the irreducible, effective divisors $S_{A}$ on which the Calabi-Yau threefold develops a singularity, while $\left[\Delta^{\prime}\right]$ is the residual class associated to singularities of the fibration which leave the total space smooth. Singularities of the Calabi-Yau threefold along $S_{A}$ corresponds to stacks of seven-branes on $S_{A}$ which admit a non-Abelian gauge theory on their world-volume. Possible gauge groups can be classified looking at the possible singularities which occur in $Y_{3}$ [16, 22, 23, 24]. The constants $\nu_{A}$ are related to group-theoretical invariants. The divisor $\Delta^{\prime}$ is wrapped by a single seven-brane with no massless gauge bosons on its world-volume. Furthermore, if $\left[\Delta^{\prime}\right]$ and some of the $\left[S_{A}\right]$ 's have non-vanishing intersection, singularity enhancements take place, which give rise to charged matter in the Type IIB picture.

In order to perform dimensional reduction of M-theory, it is necessary to resolve the Calabi-Yau threefold $Y_{3}$ if it is singular. This amounts to find a smooth Calabi-Yau threefold $\tilde{Y}_{3}$ and a map $\tilde{f}: \tilde{Y}_{3} \rightarrow Y_{3}$ such that singular loci on $Y_{3}$ are preimages through $\tilde{f}$ of so-called exceptional divisors on $\tilde{Y}_{3}$. This can be done in a canonical way, both if the singularity locus is a point and if it is a smooth curve [16, 22, 32]. The resolution procedure can be given the following physical description in the F-/M-theory duality picture. The non-Abelian gauge theory with group $G_{A}$ living on the unresolved stack of seven-branes at $\left[S_{A}\right]$ goes to its $5 \mathrm{~d}$ Coulomb branch in the resolved space, with Abelian gauge group $U(1)^{\operatorname{rank}(G)}$. Indeed, in M-theory M2-branes wrapping the $\mathbb{P}^{1}$-fibres of the exceptional divisors encode the degrees of freedom of vectors that are massive in the Coulomb branch and become massless as the exceptional divisors are shrunk to zero size. 
In the remaining part of this section we collect some results about divisors and intersection numbers of an elliptically fibred Calabi-Yau threefold 2 Let us start by considering the case of a smooth threefold $Y_{3}$. On such a space there is a natural set of divisors which span $H_{4}\left(Y_{3}, \mathbb{R}\right)$. Firstly, one has the section of the fibration which is homologous to the base $B_{2}$. Secondly, there is the set of vertical divisors $D_{\alpha}$ which are obtained as $D_{\alpha}=\pi^{-1}\left(D_{\alpha}^{\mathrm{b}}\right)$, where $D_{\alpha}^{\mathrm{b}}$ is a divisor of $B_{2}$ and $\pi$ is the projection to the base $\pi: Y_{3} \rightarrow B_{2}$. For these smooth elliptic fibrations one has $h^{1,1}\left(B_{2}\right)=h^{1,1}\left(Y_{3}\right)-1$ such divisors. Let $\omega_{0}, \omega_{\alpha}$ be the two-form cohomology classes Poincaré dual to $B_{2}, D_{\alpha}$. It is useful to record some facts concerning intersections of divisors for smooth elliptic fibrations. Due to the fibration structure one has

$$
D_{\alpha} \cap D_{\beta} \cap D_{\gamma}=0
$$

We also introduce the matrix $\eta_{\alpha \beta}$ by defining

$$
\eta_{\alpha \beta}=D_{\alpha}^{\mathrm{b}} \cap D_{\beta}^{\mathrm{b}}=B_{2} \cap D_{\alpha} \cap D_{\beta} .
$$

Note that $\eta_{\alpha \beta}$ is a non-degenerate symmetric matrix with mostly minus Lorentzian signature $\left(1, h^{1,1}\left(B_{2}\right)-1\right)$. Finally, let us recall the cohomological identity 3

$$
\omega_{0} \wedge \omega_{0}+c_{1}\left(B_{2}\right) \wedge \omega_{0}=0
$$

We also introduce the vector $K^{\alpha}$ by expanding the canonical class $[K]$ in a basis two-forms dual to vertical divisors as

$$
[K]=K^{\alpha} \omega_{\alpha} .
$$

Some basic formulas for the base $B_{2}$ of $Y_{3}$ will be useful later. The Euler number $\chi\left(B_{2}\right)$ and the integral of $c_{1}^{2}\left(B_{2}\right)$ can be generally evaluated as

$$
\chi\left(B_{2}\right)=\int_{B_{2}} c_{2}\left(B_{2}\right)=2+h^{1,1}\left(B_{2}\right), \quad \int_{B_{2}} c_{1}^{2}\left(B_{2}\right)=K^{\alpha} K^{\beta} \eta_{\alpha \beta}=10-h^{1,1}\left(B_{2}\right),
$$

where we have used $c_{1}\left(B_{2}\right)=-K^{\alpha} \omega_{\alpha}$, and the fact that $h^{1,0}\left(B_{2}\right)=h^{2,0}\left(B_{2}\right)=0$ for a base of a Calabi-Yau manifold.

Let us now take into account a singular Calabi-Yau threefold $Y_{3}$ and its resolution $\tilde{Y}_{3}$. For the sake of simplicity, we will restrict ourselves to the case of a single seven-brane stack, thus omitting the sum over index $A$ in $(2.9),[\Delta]=\nu[S]+\left[\Delta^{\prime}\right]$. Let $D_{i}$ be the exceptional divisors introduced by resolving the singularity. The index $i$ runs from 1 to $\operatorname{rank}(G)$. The cohomology class Poincaré dual to $D_{i}$ is denoted $\omega_{i}$. Furthermore, let us expand the divisor $S$ wrapped by the stack of branes in a basis two-forms dual to vertical divisors as

$$
[S]=C^{\alpha} \omega_{\alpha}
$$

\footnotetext{
${ }^{2}$ Full $S U(3)$-holonomy is always understood.

${ }^{3}$ We will be slightly sloppy with the notation in the following, since we do not explicitly indicate that certain quantities, e.g. the first Chern class $c_{1}\left(B_{2}\right)$, have to be pulled back from $B_{2}$ to the Calabi-Yau threefold.
} 
Note that, after resolution, this is replaced by

$$
[\hat{S}]=C^{\alpha} \omega_{\alpha}+a^{i} \omega_{i}
$$

where $a^{i}$ are the Dynkin numbers characterizing the Dynkin diagram of $G .4$ Exceptional divisors enjoy the following properties:

$$
\begin{aligned}
B_{2} \cap D_{i} & =0 \\
D_{\alpha} \cap D_{i} \cap D_{j} & =-C_{i j} B_{2} \cap D_{\alpha} \cap S \\
D_{\alpha} \cap D_{\beta} \cap D_{i} & =0,
\end{aligned}
$$

where $C_{i j}$ is the Cartan matrix of the group $G$.

We are now in a position to summarize all intersection numbers on the resolved Calabi-Yau threefold $\tilde{Y}_{3}$. We have found a cohomology basis $\left\{\omega_{0}, \omega_{\alpha}, \omega_{i}\right\}$ which can be denoted collectively as $\left\{\omega_{\Lambda}\right\}$. Intersection numbers are defined as

$$
\mathcal{V}_{\Lambda \Sigma \Theta}=\int_{\tilde{Y}_{3}} \omega_{\Lambda} \wedge \omega_{\Sigma} \wedge \omega_{\Theta}
$$

Identities and properties listed above imply that intersection numbers must satisfy

$$
\begin{array}{rlrl}
\mathcal{V}_{000} & =\eta_{\alpha \beta} K^{\alpha} K^{\beta} & \mathcal{V}_{0 i \Lambda} & =0 \\
\mathcal{V}_{00 \alpha} & =\eta_{\alpha \beta} K^{\beta} & & \mathcal{V}_{\alpha i j}=-C_{i j} \eta_{\alpha \beta} C^{\beta} \\
\mathcal{V}_{0 \alpha \beta} & =\eta_{\alpha \beta} & & \mathcal{V}_{\alpha \beta i}=0 \\
\mathcal{V}_{\alpha \beta \gamma} & =0, &
\end{array}
$$

where $\Lambda=0, \alpha, j$. As far as $\mathcal{V}_{i j k}$ is concerned, in general it is non-vanishing, but otherwise unconstrained by our discussion so far. These intersection numbers arise from intersecting exceptional divisors. In fact, as we will discuss below, they will be linked to grouptheoretical factors depending on the charged matter content of the gauge theory.

\section{Circle compactification from six to five dimensions}

In this section we discuss the circle reduction of a general $6 \mathrm{~d}(1,0)$ supergravity theory. After reviewing some foundational material about $6 \mathrm{~d}$ supergravities with a simple non-Abelian gauge group in subsection 3.1, the details of the dimensional reduction are presented in subsection 3.2 supplemented by appendix $\mathrm{C}$. We emphasize the treatment of self-dual two-forms, and describe both the reduction of the non-Abelian gauge theory

\footnotetext{
${ }^{4}$ Note that after singularity resolution also (2.13) is modified by the addition of non-trivial $\omega_{i}$ terms. Nonetheless, these terms do not affect the following discussion on intersection numbers, thanks to identities 2.17)
} 
and its broken phase relevant in the match with M-theory. The $5 \mathrm{~d}$ action is brought into canonical $\mathcal{N}=2$ form in subsection 3.4. We point out an intriguing generalization of the $\mathcal{N}=2$ formalism which captures the full reduced action. In subsection 3.5 certain higher order curvature corrections are reduced which carry crucial information about gravitational $6 \mathrm{~d}$ anomalies.

\subsection{Generalities on $6 d(1,0)$ supergravity}

In this subsection we review some basic facts about the spectrum and the dynamics of a generic $6 \mathrm{~d}$ supergravity model with $(1,0)$ supersymmetry, corresponding to 8 real supercharges. Massless states in six dimensions are classified by representations of the little group $S O(4) \cong S U(2) \times S U(2)$ and are therefore labelled by a couple of integer or half-integer spins, $\left(j_{L}, j_{R}\right)$. Four different kinds of supersymmetric multiplets can be constructed, restricting to spin less or equal to two [14]. We list them following the chirality conventions which are more common in the $6 \mathrm{~d}$ supergravity literature, cf. e.g. [5]:

- gravity multiplet: $(1,1) \oplus 2\left(\frac{1}{2}, 1\right) \oplus(1,0)$, i.e. the graviton, one Weyl 5 left-handed gravitino, one self-dual two-form;

- vector multiplet: $\left(\frac{1}{2}, \frac{1}{2}\right) \oplus 2\left(\frac{1}{2}, 0\right)$, i.e. one vector and one Weyl left-handed gaugino;

- tensor multiplet: $(0,1) \oplus 2\left(0, \frac{1}{2}\right) \oplus(0,0)$, i.e. one anti-self-dual two-form, one Weyl right-handed tensorino, one real scalar;

- hypermultiplet: $2\left(0, \frac{1}{2}\right) \oplus 4(0,0)$, i.e. one Weyl right-handed hyperino and two complex scalars.

A general model features one gravity multiplet, $n_{V}$ vector multiplets, $n_{H}$ hypermultiplets, $n_{T}$ tensor multiplets. It is well known that the (anti-)self-duality condition is incompatible with a naïve Lagrangian formulation, because the usual kinetic term for two-forms vanishes identically once it is taken into account. In the special case $n_{T}=1$, the anti-self-dual two-form from the gravity multiplet and the self-dual two-form from the tensor multiplet can be combined into a two-form without any self-duality property, and the standard Lagrangian formulation applies. Nonetheless, a set of consistent, supersymmetric, two-derivative, classical equations of motion is known for arbitrary $n_{T}$ [5]. We can still derive them from variation of a suitable functional of the fields (called pseudo-action), provided that the self-duality condition is imposed after computation of functional derivatives. In this paper, all $6 \mathrm{~d}$ actions are to be interpreted in this weak sense. 6

\footnotetext{
${ }^{5}$ An equivalent formulation makes use of a $S U(2)$ doublet of Weyl left-handed gravitini $(S U(2)$ is the automorphism group of the supersymmetry algebra), supplemented by a symplectic Majorana condition. Similar remarks apply to all other fermions. This explains why this model is sometimes referred to as $\mathcal{N}=2$ in the literature.

${ }^{6}$ This formalism is usually applied to Type IIB supergravity in ten dimensions to deal with the self-dual four-form in the RR sector.
} 
We will always restrict ourselves to the bosonic content of the model, and adopt notations described below. First of all, we denote all $6 \mathrm{~d}$ two-forms collectively as $\hat{B}^{\alpha}$, where $\alpha=1, \ldots n_{T}+17$ The scalars coming from the $n_{T}$ tensor multiplets parameterize the quotient

$$
S O\left(1, n_{T}\right) / S O\left(n_{T}\right)
$$

It is customary to describe this coset scalar manifold by means of a vielbein formalism. We refer the reader to e.g. [5] for a detailed account. For our present discussion we need only to recall that a constant $S O\left(1, n_{T}\right)$ metric $\Omega_{\alpha \beta}$ is introduced, along with a set of $n_{T}+1$ scalar fields $j^{\alpha}$. The metric $\Omega_{\alpha \beta}$ has mostly minus Lorentzian signature $\left(1, n_{T}\right)$, and the scalars $j^{\alpha}$ are subject to the constraint

$$
\Omega_{\alpha \beta} j^{\alpha} j^{\beta}=1
$$

Moreover, the scalar manifold is endowed with another non-constant, positive definite metric $g_{\alpha \beta}$, which is given in terms of $\Omega_{\alpha \beta}, j^{\alpha}$ by

$$
g_{\alpha \beta}=2 j_{\alpha} j_{\beta}-\Omega_{\alpha \beta}
$$

where $j_{\alpha}=\Omega_{\alpha \beta} j^{\beta}$. This metric is needed to write down the (anti)-self-duality condition for $\hat{B}^{\alpha}$ in a $S O\left(1, n_{T}\right)$ covariant way, as we will see in equation (3.21).

As far as vectors are concerned, in this section we consider a supergravity model with simple gauge group $G$. Let $\mathfrak{g}$ be the Lie algebra of $G$. We denote the $\mathfrak{g}$-valued gauge one-form by $\hat{A}$, and matrix multiplication will always be understood. Moreover, we use anti-Hermitian generators, and the expression for the non-Abelian field strength two-form reads

$$
\hat{F}=d \hat{A}+\hat{A} \wedge \hat{A}=d \hat{A}+\frac{1}{2}[\hat{A}, \hat{A}] .
$$

Let us recall the definition of the Chern-Simons three-form

$$
\hat{\omega}^{\mathrm{CS}}=\operatorname{tr}\left(\hat{A} \wedge d \hat{A}+\frac{2}{3} \hat{A} \wedge \hat{A} \wedge \hat{A}\right)
$$

where the trace is taken in a suitable representation of $\mathfrak{g}$. More details about our normalization for gauge traces can be found in appendix B. It is also useful to point out two key properties of the Chern-Simons three-form,

$$
\delta \hat{\omega}^{\mathrm{CS}}=\operatorname{tr} d \hat{\lambda} \wedge d \hat{A}, \quad d \hat{\omega}^{\mathrm{CS}}=\operatorname{tr} \hat{F} \wedge \hat{F} .
$$

Next, let us make some remarks about the hyper sector. Each hypermultiplet contains four real scalars, and therefore we use the notation $q^{U}\left(U=1, \ldots, 4 n_{H}\right)$. These scalar fields can be considered as real coordinates for a quaternionic manifold, whose metric we write as $h_{U V}$. The geometric structures of quaternionic manifolds have been studied intensively, see e.g. [33, 34]. Since our main focus will be on the tensor and vector multiplet structure,

\footnotetext{
${ }^{7}$ Later on we will identify $n_{T}+1=h^{1}, 1\left(B_{2}\right)$ in the duality to M-theory. This provides the match of the indices of the present section with the ones of section 2.2
} 
we will refrain from giving a detailed account of these results here. However, in the following we will need to consider some aspects of charged hypermultiplets. The only piece of information relevant to our discussion is the $6 \mathrm{~d}$ covariant derivative, which reads schematically

$$
\hat{\mathcal{D}} q^{U}=d q^{U}+\hat{A}^{I}\left(T_{I}^{\mathbf{R}} q\right)^{U}
$$

where the index $I$ runs over all generators of the gauge group $G$, and $T_{I}^{\mathbf{R}}$ are the group generators acting on the scalars $q^{U}$ in the representation $\mathbf{R}$. Several examples of gauged 6d $(1,0)$ supergravities are known. We refer the reader to [35, 36, 37, 38] and references therein for a detailed account on the subject.

Finally, gravitational degrees of freedom are described by means of the vielbein formalism. The analogue of the one-form gauge connection $\hat{A}$ is provided by the $\mathfrak{s o}(1,5)$-valued spin connection one-form $\hat{\omega}$, determined by the vielbein through the usual torsionless condition

$$
d \hat{e}+\hat{\omega} \wedge \hat{e}=0,
$$

where matrix multiplication is understood. If $\hat{\ell}$ is a $\mathfrak{s o}(1,5)$-valued zero-form which we interpret as infinitesimal parameter of a local Lorentz transformation, we have

$$
\delta \hat{\omega}=d \hat{\ell}+[\hat{\omega}, \hat{\ell}] .
$$

The correct covariant field strength is the curvature two-form $\hat{\mathcal{R}}$, which is constructed out of the spin connection according to

$$
\hat{\mathcal{R}}=d \hat{\omega}+\hat{\omega} \wedge \hat{\omega}
$$

and is related to the components of the $6 \mathrm{~d}$ Riemann tensor $\hat{R}_{\hat{\tau} \hat{\mu} \hat{\mu}}^{\hat{\nu}}$ by

$$
\hat{\mathcal{R}}^{\hat{a}}{ }_{\hat{b}}=\frac{1}{2} \hat{e}_{\hat{\lambda}}^{\hat{a}} \hat{e}_{\hat{b}}^{\hat{\tau}} \hat{R}_{\hat{\tau} \hat{\mu} \hat{\nu}}^{\hat{\lambda}} d \hat{x}^{\hat{\mu}} \wedge d \hat{x}^{\hat{\nu}}, \quad \hat{a}, \hat{b},=0, \ldots, 5
$$

We also define a gravitational Chern-Simons three-form

$$
\hat{\omega}_{\text {grav }}^{\mathrm{CS}}=\operatorname{tr}\left(\hat{\omega} \wedge d \hat{\omega}+\frac{2}{3} \hat{\omega} \wedge \hat{\omega} \wedge \hat{\omega}\right) .
$$

This definition implies the identities

$$
\delta \hat{\omega}_{\text {grav }}^{\mathrm{CS}}=\operatorname{tr} d \hat{\ell} \wedge d \hat{\omega}, \quad d \hat{\omega}_{\text {grav }}^{\mathrm{CS}}=\operatorname{tr} \hat{\mathcal{R}} \wedge \hat{\mathcal{R}} .
$$

Note that the right hand side of the last equation is proportional to a characteristic class build from the curvature two-form. In general, the proportionality constant is fixed by the requirement that suitable integrals of such classes take integer values. This standard normalization is achieved by inserting a factor of $(2 \pi)^{-1}$ for each occurrence of the curvature two-form $\hat{\mathcal{R}}$ specified by (3.11). In order to improve readability, we will never write down these factors of $(2 \pi)^{-1}$ in the following. Similar remarks apply to the $5 \mathrm{~d}$ curvature two-form introduced in section 3.2 .

As we have seen above, the spectrum of a general $6 \mathrm{~d}(1,0)$ supergravity model contains chiral fermions and (anti)-self-dual two-forms. As a result, gauge, gravitational, and 
mixed anomalies may appear once one-loop effects are taken into account. Nonetheless, a generalization of 10d Green-Schwarz mechanism, due to Sagnotti [12, 13, 18], can be implemented to generate consistent, anomaly-free theories: it is reviewed concisely in appendix B. Let us just recall now that, under suitable conditions on the matter content of the model, the anomaly polynomial factorizes,

$$
\hat{I}_{8}=\frac{1}{2} \Omega_{\alpha \beta} \hat{X}_{4}^{\alpha} \wedge \hat{X}_{4}^{\beta}
$$

where

$$
\hat{X}_{4}^{\alpha}=\frac{1}{2} a^{\alpha} \operatorname{tr} \hat{\mathcal{R}} \wedge \hat{\mathcal{R}}+2 b^{\alpha} \operatorname{tr} \hat{F} \wedge \hat{F} .
$$

If this is the case, even if $\hat{I}_{8}$ is non-vanishing, anomalies can be counterbalanced by adding the so-called Green-Schwarz term to the action,

$$
\hat{S}^{\mathrm{GS}}=-\frac{1}{2} \int_{\mathcal{M}_{6}} \Omega_{\alpha \beta} \hat{B}^{\alpha} \wedge \hat{X}_{4}^{\beta}
$$

In order for this generalized Green-Schwarz mechanism to work, we have to assign the following non-trivial transformation rules to the fields of the model:

$$
\begin{aligned}
\delta \hat{A} & =d \hat{\lambda}+[\hat{A}, \hat{\lambda}] \\
\delta \hat{B}^{\alpha} & =d \hat{\Lambda}^{\alpha}-\frac{1}{2} a^{\alpha} \operatorname{tr} \hat{\ell} d \hat{\omega}-2 b^{\alpha} \operatorname{tr} \hat{\lambda} d \hat{A} .
\end{aligned}
$$

In the second equation, $\Lambda^{\alpha}$ is a collection of one-forms which are the parameters of the usual Abelian gauge invariance of two-form potentials. The correct, gauge-invariant field strength three-form for $\hat{B}^{\alpha}$ turns out to be

$$
\hat{G}^{\alpha}=d \hat{B}^{\alpha}+\frac{1}{2} a^{\alpha} \hat{\omega}_{\text {grav }}^{\mathrm{CS}}+2 b^{\alpha} \hat{\omega}^{\mathrm{CS}},
$$

and satisfies a non-standard Bianchi identity,

$$
d \hat{G}^{\alpha}=\hat{X}_{4}^{\alpha}
$$

The self-duality constraint for the two-forms is written in terms of the three-form field strengths as

$$
g_{\alpha \beta} \hat{*} \hat{G}^{\alpha}=\Omega_{\alpha \beta} \hat{G}^{\beta},
$$

where $g_{\alpha \beta}$ is the positive-definite, non-constant metric introduced in (3.3)).

We are now in a position to write down the pseudo-action for $6 \mathrm{~d}(1,0)$ supergravity with simple gauge group $G$. Its purely bosonic terms relevant to us are given by

$$
\begin{aligned}
\hat{S}^{(6)}=\int_{\mathcal{M}_{6}} & +\frac{1}{2} \hat{R} \hat{*} 1-h_{U V} \hat{\mathcal{D}} q^{U} \wedge * \hat{\mathcal{D}} q^{V}-\frac{1}{4} g_{\alpha \beta} \hat{G}^{\alpha} \wedge \hat{*} \hat{G}^{\beta}-\frac{1}{2} g_{\alpha \beta} d j^{\alpha} \wedge \hat{*} d j^{\beta} \\
& -2 \Omega_{\alpha \beta} j^{\alpha} b^{\beta} \operatorname{tr} \hat{F} \wedge \hat{*} \hat{F}-\frac{1}{2} \Omega_{\alpha \beta} \hat{B}^{\alpha} \wedge \hat{X}_{4}^{\beta}-\hat{V} \hat{*} 1
\end{aligned}
$$

In the second line, $\hat{V}$ is a potential generated by gauging the hypermultiplet scalars $q^{U}$. Its explicit form can be found e.g. in [38], but will not be crucial for our discussion. Recall 
that this action has to be supplemented by the duality constraint (3.21) imposed on the level of the equations of motion. Note that the second-order equation obtained through variation of $\hat{B}^{\alpha}$ is equivalent to the exterior derivative of (3.21) thanks to (3.20). This action contains a two-derivative part which yields the equations of motion discussed in [6]. We included in (3.22) one additional higher derivative term which is the generalized Green-Schwarz term (3.16) required for 6d anomaly cancellation.

It is appropriate to point out that the Green-Schwarz term is a possible source of non gauge-invariance of this classical action. Indeed, one computes

$$
\delta \hat{S}^{(6)}=\frac{1}{2} \int_{\mathcal{M}_{6}} \Omega_{\alpha \beta}\left(\frac{1}{2} a^{\alpha} \operatorname{tr} \hat{\ell} d \hat{\omega}+2 b^{\alpha} \operatorname{tr} \hat{\lambda} d \hat{A}\right) \wedge \hat{X}_{4}^{\beta},
$$

which in general is not just a surface contribution. It is precisely this failure of gauge invariance at tree-level which cancels one-loop anomalies. We summarize the anomaly conditions in appendix B. For completeness let us point out that there is a simple special case where the action is already classically gauge invariant. It is enforced by the conditions

$$
\Omega_{\alpha \beta} a^{\alpha} a^{\beta}=0, \quad \Omega_{\alpha \beta} a^{\alpha} b^{\beta}=0, \quad \Omega_{\alpha \beta} b^{\alpha} b^{\beta}=0 .
$$

These conditions on $a^{\alpha}, b^{\alpha}$ can be related to the spectrum of fields, in particular the charge matter content, through the anomaly cancellation conditions (B.4 $)(\overline{B .9})$ of appendix B. As we argue in section 5, the match between the F-theory set-up and the M-theory compactification is simpler in this special case.

\subsection{Kaluza-Klein reduction on the circle}

Let us now study the supergravity model outlined above on a background with one compact spatial dimension, i.e. with topology $\mathbb{R}^{5} \times S^{1}$. Degrees of freedom along the circle can be analysed in terms of their Fourier expansion, giving rise to an infinite tower of Kaluza-Klein modes. As discussed above, we restrict ourselves to zero-modes only.

Our metric Ansatz reads

$$
d \hat{s}_{(6)}^{2}=\tilde{g}_{\mu \nu} d x^{\mu} d x^{\nu}+r^{2} D y^{2}, \quad D y=d y-A^{0},
$$

where $A^{0}=A_{\mu}^{0} d x^{\mu}$, and all $5 \mathrm{~d}$ field are independent of the coordinate $y$ along $S^{1}$. A twiddle is used to stress that this form of the metric gives rise to a non-canonically normalized action for gravity, so that a Weyl rescaling has to be performed. As usual, $A^{0}$ is a 5 d vector with Abelian $U(1)$ symmetry $A^{0} \rightarrow A^{0}+d \chi$ coming from $S^{1}$ diffeomorphisms $y \rightarrow y+\chi$, which leave the derivative $D y$ invariant. The field strength of $A^{0}$ reads

$$
F^{0}=d A^{0} .
$$

It is useful to write down the Kaluza-Klein Ansatz for the metric in the vielbein formalism, too. Up to local Lorentz transformations, we can take

$$
\hat{e}^{a}=\tilde{e}_{\mu}^{a} d x^{\mu}, \quad \hat{e}^{5}=r D y,
$$


where $D y$ is given in (3.25), and $\tilde{e}_{\mu}^{a}, a=0, \ldots, 4$ is the $5 \mathrm{~d}$ vielbein (independent of $y$ ) before Weyl rescaling.

Let us now turn to the one-forms and two-forms, and take into account zero-modes only. In order to get $5 \mathrm{~d}$ fields which are uncharged under the aforementioned $U(1)$ symmetry, we expand all fields on Dy defined in (3.25). To begin with, we set

$$
\hat{A}=A+\zeta D y
$$

where $\zeta$ is a $\mathfrak{g}$-valued $5 \mathrm{~d}$ zero-form. The gravitational analogue of this relation consists of the expression for the spin connection components, which can be computed from (3.27):

$$
\hat{\omega}_{a b}=\tilde{\omega}_{a b}+\tilde{\mathfrak{a}}_{a b}^{(0)} D y, \quad \hat{\omega}_{a 5}=\tilde{\mathfrak{b}}_{a}^{(1)}+\tilde{\mathfrak{c}}_{a}^{(0)} D y,
$$

where $\tilde{\boldsymbol{\omega}}$ is the $5 \mathrm{~d}$ spin connection determined by $\tilde{e}_{\mu}^{a}$. The zero-forms $\tilde{\mathfrak{a}}_{a b}^{(0)}, \tilde{\mathfrak{c}}_{a}^{(0)}$, and the one-form $\tilde{\mathfrak{b}}_{a}^{(1)}$ are given by

$$
\tilde{\mathfrak{a}}_{a b}^{(0)}=\frac{1}{2} r^{2} \tilde{e}_{a}^{\mu} \tilde{e}_{b}^{\nu} F_{\mu \nu}^{0}, \quad \tilde{\mathfrak{b}}_{a}^{(1)}=\frac{1}{2} r \tilde{e}_{a}^{\lambda} F_{\lambda \mu}^{0} d x^{\mu}, \quad \tilde{\mathfrak{c}}_{a}^{(0)}=-\tilde{e}_{a}^{\lambda} \tilde{\nabla}_{\lambda} r,
$$

where $\tilde{\nabla}_{\lambda}$ is the 5 d Levi-Civita connection before Weyl rescaling.

We are now in a position to write down the Kaluza-Klein Ansatz for the two-forms $\hat{B}^{\alpha}$. Care has to be taken because the $6 \mathrm{~d}$ transformation rule (3.18) entangles the degrees of freedom encoded in $\hat{B}^{\alpha}$ with those of vectors and gravity. Thus, we set

$$
\hat{B}^{\alpha}=B^{\alpha}-\left[A^{\alpha}-\frac{1}{2} a^{\alpha} \operatorname{tr}\left(\tilde{\mathfrak{a}}^{(0)} \tilde{\omega}\right)-2 b^{\alpha} \operatorname{tr}(\zeta A)\right] \wedge D y .
$$

In this way $A^{\alpha}, B^{\alpha}$ have the simplest possible gauge transformations,

$$
\begin{aligned}
& \delta A^{\alpha}=d \mu^{\alpha} \\
& \delta B^{\alpha}=d \Lambda^{\alpha}+\mu^{\alpha} F^{0}-\frac{1}{2} a^{\alpha} \operatorname{tr}(\ell d \tilde{\omega})-2 b^{\alpha} \operatorname{tr}(\lambda d A),
\end{aligned}
$$

where the infinitesimal parameters are a $\mathfrak{g}$-valued $5 \mathrm{~d}$ zero-form $\lambda$, a $\mathfrak{s o}(1,4)$-valued $5 \mathrm{~d}$ zero-form $\ell, 5 \mathrm{~d}$ zero-, one-forms $\mu^{\alpha}, \Lambda^{\alpha}$. The first relation implies that $A^{\alpha}$ has a standard, Abelian field strength

$$
F^{\alpha}=d A^{\alpha}
$$

However, the naïve field strength $d B^{\alpha}$ is not gauge invariant, and must be improved by setting

$$
G^{\alpha}=d B^{\alpha}-A^{\alpha} \wedge F^{0}+\frac{1}{2} a^{\alpha} \tilde{\omega}_{\mathrm{grav}}^{\mathrm{CS}}+2 b^{\alpha} \omega^{\mathrm{CS}},
$$

where

$$
\begin{aligned}
\tilde{\omega}_{\text {grav }}^{\mathrm{CS}} & =\operatorname{tr}\left(\tilde{\omega} \wedge d \tilde{\omega}+\frac{2}{3} \tilde{\omega} \wedge \tilde{\omega} \wedge \tilde{\omega}\right), \\
\omega^{\mathrm{CS}} & =\operatorname{tr}\left(A \wedge d A+\frac{2}{3} A \wedge A \wedge A\right) .
\end{aligned}
$$

The corresponding non-standard Bianchi identity reads

$$
d G^{\alpha}=-F^{\alpha} \wedge F^{0}+\frac{1}{2} a^{\alpha} \operatorname{tr} \tilde{\mathcal{R}} \wedge \tilde{\mathcal{R}}+2 b^{\alpha} \operatorname{tr} F \wedge F .
$$


In the rest of this subsection, we will only focus on the two-derivative Lagrangian. As a consequence, we drop higher curvature terms from the $6 \mathrm{~d}$ pseudo-action, and we also neglect gravitational contribution to the gauge transformation of $B^{\alpha}$ and to the field strength $G^{\alpha}$. A discussion of the higher curvature corrections can be found in subsection 3.5 .

Dimensional reduction of action (3.22) is performed in appendix $\mathrm{C}$, to which we refer the reader for more details. However, let us just stress here that the resulting $5 \mathrm{~d}$ action is a proper action, without any need for auxiliary self-duality conditions. This is possible because the $6 \mathrm{~d}$ two-forms $\hat{B}^{\alpha}$ dimensionally reduce to two-forms $B^{\alpha}$ and vectors $A^{\alpha}$ as seen in (3.31). At the same time, we also have to dimensionally reduce the self-duality constraint (3.21). Explicitly we find

$$
r g_{\alpha \beta} \tilde{*} G^{\beta}=-\Omega_{\alpha \beta} \mathcal{F}^{\beta}
$$

where we have introduced the shorthand notation

$$
\mathcal{F}^{\alpha}=F^{\alpha}-4 b^{\alpha} \operatorname{tr}(\zeta F)+2 b^{\alpha} \operatorname{tr}(\zeta \zeta) F^{0} .
$$

The key point is that the 5d duality condition (3.39) now relates two-forms and vectors. Since it does not involve a self-duality, it can be imposed on the level of the action itself. Hence, in computing the $5 \mathrm{~d}$ action we proceed in the two steps:

1. We rewrite the $5 \mathrm{~d}$ pseudo-action $S_{\text {pseudo }}^{(5) \mathrm{F}}$ resulting from reduction of (3.22) in a form such that $B^{\alpha}$ only appears through its field strength $G^{\alpha}$. Moreover, $G^{\alpha}$ can be treated as an independent variable which enters the action only algebraically.

2. The 5 d pseudo-action $S_{\text {pseudo }}^{(5) \mathrm{F}}$ can be replaced by an actual action by adding terms of the schematic form $\Omega_{\alpha \beta} d B^{\alpha} \wedge F^{\beta}$ to the action to impose the condition (3.39). More precisely, the modification is of the form

$$
\Delta S^{(5) \mathrm{F}}=-\int_{\mathcal{M}_{5}} \frac{1}{2} \Omega_{\alpha \beta}\left(G^{\alpha}+A^{\alpha} \wedge F^{0}-2 b^{\alpha} \omega^{\mathrm{CS}}\right) \wedge F^{\beta} .
$$

The first term proportional to $G^{\alpha}$ acts as Lagrangian multiplier term to link $G^{\alpha}$ with its dual $\mathcal{F}^{\alpha}$. The remaining two terms act as source terms which ensure compatibility with the modified Bianchi identity (3.38) of $G^{\alpha}$. Including these modifications, both the self-duality constraint and the Bianchi identity for $G^{\alpha}$ follow from the equations of motion. We are thus able to integrate $G^{\alpha}$ out and obtain a $5 \mathrm{~d}$ proper action $S^{(5) \mathrm{F}}$, written in terms of the vectors $A^{\alpha}$ only.

The 5d action which results from this algorithm can be found in (C.8). It is interesting to note that these two steps can be performed even if we reintroduce the gravitational part of the generalized Green-Schwarz term, and all gravitational contributions to $G^{\alpha}$, as discussed in section 3.5 . 


\subsection{Moving to the Coulomb branch}

In the following sections, we will explore the dynamics of F-theory in six dimensions by means of the duality with M-theory on a Calabi-Yau threefold, as introduced in section 2. In this framework, we can access directly only the Coulomb branch of our non-Abelian gauge sector. The full gauge group $G$ is spontaneously broken down to $U(1)^{\operatorname{rank}(G)}$, which is spanned by the Cartan generators $T_{i}, i=1, \ldots, \operatorname{rank}(G)$. We take them to be normalized in such a way that

$$
\operatorname{tr}\left(T_{i} T_{j}\right)=C_{i j}
$$

where $C_{i j}$ is the Cartan matrix of $G$.

The spontaneous break down of gauge symmetry is triggered by non-vanishing VEVs of some adjoint scalars $\zeta$ in the vector multiplets. In particular, inspection of the terms

$$
-2 r^{2 / 3} \Omega_{\alpha \beta} j^{\alpha} b^{\beta} \operatorname{tr} F \wedge * F-2 r^{-2} \Omega_{\alpha \beta} j^{\alpha} b^{\beta} \operatorname{tr} D \zeta \wedge * D \zeta
$$

in the non-Abelian 5d action (C.8) shows that the usual Higgs mechanism originates a mass term for the vectors lying outside of the Cartan subalgebra. We refer to these massive vectors as $\mathrm{W}$-bosons. Their scalar partners acquire a mass, as well. From an effective field theory perspective, we are thus left only with the massless fields $A^{i}, \zeta^{i}$ associated to the Cartan subalgebra of the full gauge algebra. As a result, replacements such as

$$
\begin{gathered}
\operatorname{tr}(F \wedge * F) \rightarrow C_{i j} F^{i} \wedge * F^{j}, \quad \operatorname{tr}(D \zeta \wedge * D \zeta) \rightarrow C_{i j} d \zeta^{i} \wedge * d \zeta^{j} \\
\omega^{\mathrm{CS}} \rightarrow C_{i j} A^{i} \wedge F^{j}
\end{gathered}
$$

have to be made in (C.8) to get the relevant 5 d action.

In a similar fashion, charged hypermultiplets acquire a mass through the $5 \mathrm{~d}$ scalar potential

$$
V=r^{-1} \hat{V}+r^{-8 / 3} h_{U V} \zeta^{I} \zeta^{J}\left(T_{I}^{\mathbf{R}} q\right)^{U}\left(T_{J}^{\mathbf{R}} q\right)^{V}
$$

given in the last line of (C.8). Note that the second term originates directly from dimensional reduction of the $6 \mathrm{~d}$ kinetic term $h_{U V} \hat{\mathcal{D}} q^{U} \wedge \hat{*} \hat{\mathcal{D}} q^{V}$. It is quadratic in the scalars of the charged hypermultiplets and is the source for their masses once gauge symmetry is spontaneously broken. Following the effective field theory paradigm, one should integrate out the massive hypermultiplets and only keep neutral hypermultiplets in the $5 \mathrm{~d}$ action in the Coulomb branch. We use lower-case indices $u, v=1, \ldots, 4 n_{H}^{\text {neutral }}$ to enumerate them. Hence, we have the replacement rule

$$
h_{U V} \mathcal{D} q^{U} \wedge * \mathcal{D} q^{V} \rightarrow h_{u v} d q^{u} \wedge * d q^{v}
$$

where $h_{u v}$ is a quantum corrected hypermultiplet metric. Determining $h_{u v}$ after integrating out the massive states is in general a complicated task, but we will later give the M-theory expression for $h_{u v}$ where certain corrections have been taken into account implicitly via the geometry. In accord with supersymmetry we also drop the scalar potential from the effective action for the massless modes. 
The interested reader can find the explicit expression for the effective action in the Coulomb branch in (C.9). However, it is crucial to recast this result in a more transparent form in order to implement the F-theory lift discussed in section 5. The aim of the following section is precisely the reformulation of the $5 \mathrm{~d}$ action in terms of new variables, in such a way to exploit the underlying supersymmetric structure. Hence, we begin our analysis with a concise review of $5 \mathrm{~d} \mathcal{N}=2$ supergravity.

\subsection{The 5d effective action and its canonical form}

Let us briefly recall the field content of $5 \mathrm{~d} \mathcal{N}=2$ ( 8 real supercharges) supersymmetry multiplets [39]:

- gravity multiplet: the graviton, one vector (referred to as 'graviphoton'), one Dirac 8 gravitino;

- vector multiplet: one vector, one scalar, one Dirac gaugino;

- hypermultiplet: 2 complex scalars, one Dirac hyperino.

Let the spectrum consist of the gravity multiplet, $n_{V}^{(5)}$ vector multiplets, $n_{H}^{(5)}$ hypermultiplets, and let us focus on the bosonic sector. We are not going to study gauged supergravity models, and therefore the framework outlined in [40] is general enough for our purposes 9. As usual, each hypermultiplet contributes four real scalars to the spectrum, and we will use notation $q^{u}$ with $u=1, \ldots, 4 n_{H}^{(5)}$. The hypersector is entirely specified once a quaternionic structure with metric $h_{u v}$ is given. Since the graviphoton and the vectors from the vector multiplets are naturally entangled by the dynamics of the theory, let us denote them collectively as $A^{\mathcal{I}}$ where $\mathcal{I}=0, \ldots, n_{V}^{(5)}$. The scalars coming from the vector multiplets parameterize a $n_{V}^{(5)}$ manifold which is most conveniently described in terms of so-called very special coordinates $M^{\mathcal{I}}$. These are $n_{V}^{(5)}+1$ real coordinates which describe an auxiliary $\left(n_{V}^{(5)}+1\right)$-dimensional manifold in which the actual scalar manifold is embedded as an hypersurface, as explained below.

The dynamics of gravity-vector sector at two-derivative level is entirely specified once the cubic potential

$$
\mathcal{N}=\frac{1}{3 !} C_{\mathcal{I} \mathcal{J K}} M^{\mathcal{I}} M^{\mathcal{J}} M^{\mathcal{K}}
$$

is given in terms of very special coordinates and of a constant symmetric tensor $C_{\mathcal{I} \mathcal{J K}}$. First of all, the scalar manifold is identified with the hypersurface described by the socalled very special geometry constraint

$$
\mathcal{N}=1 .
$$

\footnotetext{
${ }^{8}$ It is customary to replace one Dirac fermion by a $S U(2)$ doublet of Dirac fermions satisfying a symplectic Majorana condition. This explains the notation $\mathcal{N}=2$.

${ }^{9}$ In order to compare formulae below with the reference, the reader should be aware that we have

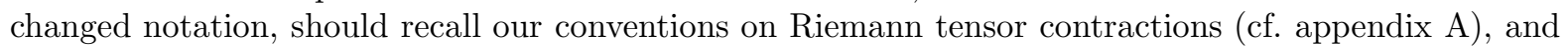
should also note that $C_{\mathcal{I} \mathcal{J K}}^{\text {there }}=\frac{\sqrt{6}}{8} C_{\mathcal{I}}^{\text {here }}$.
} 
Second of all, the gauge coupling function and the metric on the scalar manifold coincide and are constructed out of second derivatives of the cubic potential,

$$
G_{\mathcal{I} \mathcal{J}}=\left[-\frac{1}{2} \partial_{M^{\mathcal{I}}} \partial_{M^{\mathcal{J}}} \log \mathcal{N}\right]_{\mathcal{N}=1}=\left[-\frac{1}{2} \mathcal{N}_{\mathcal{I} \mathcal{J}}+\frac{1}{2} \mathcal{N}_{\mathcal{I}} \mathcal{N}_{\mathcal{J}}\right]_{\mathcal{N}=1}
$$

In this expression, and in the following, downstairs indices $\mathcal{I}, \mathcal{J}, \ldots$ denote partial derivative with respect to coordinates $N^{\mathcal{I}}, M^{\mathcal{J}}, \ldots$

Finally, the constant tensor $C_{\mathcal{I} \mathcal{J K}}$ itself appears in the action as Chern-Simons coupling. Indeed, the action is given by

$$
\begin{aligned}
S^{(5) \text { can }}=\int_{\mathcal{M}_{5}} & +\frac{1}{2} R * 1-\frac{1}{2} G_{\mathcal{I} \mathcal{J}} d M^{\mathcal{I}} \wedge * d M^{\mathcal{J}}-h_{u v} d q^{u} \wedge * d q^{v} \\
& -\frac{1}{2} G_{\mathcal{I} \mathcal{J}} F^{\mathcal{I}} \wedge * F^{\mathcal{J}}-\frac{1}{12} C_{\mathcal{I} \mathcal{J} \mathcal{K}} A^{\mathcal{I}} \wedge F^{\mathcal{J}} \wedge F^{\mathcal{K}} .
\end{aligned}
$$

Let us now discuss the relation between the spectrum of a $6 \mathrm{~d}$ supergravity model and the spectrum of its Kaluza-Klein reduction on a circle. Suppose the numbers of $6 \mathrm{~d}$ tensor, vector and hypermultiplets are $n_{T}, n_{V}, n_{H}$ respectively. To begin with, we note that the bosonic part of a hypermultiplet behaves trivially under dimensional reduction on $S^{1}$. Hence, we can conclude that the number $n_{H}^{(5)}$ of 5 d hypermultiplets is given simply by

$$
n_{H}^{(5)}=n_{H}^{\text {neutral }}
$$

where the label 'neutral' has been added to remind the reader that charged $6 \mathrm{~d}$ hypermultiplets are integrated out and do not appear in the $5 \mathrm{~d}$ effective theory.

As far as 5 d vectors are concerned, they are generated by three different mechanisms. First of all, one vector $A^{0}$ is introduced by the off-diagonal component of the Kaluza-Klein Ansatz for the $6 \mathrm{~d}$ metric. Second of all, $n_{T}+1$ vectors $A^{\alpha}$ come from the (anti)-selfdual two-forms in six-dimensions. Finally, reduction of $6 \mathrm{~d}$ vectors gives us $n_{V}$ additional $A^{i}$. We thus have a total of $1+\left(n_{T}+1\right)+n_{V}$ vectors, which we denote collectively as $A^{\mathcal{I}}=\left(A^{0}, A^{\alpha}, A^{i}\right)$. They fit into

$$
n_{V}^{(5)}=n_{V}+n_{T}+1
$$

$5 \mathrm{~d}$ vector multiplets, because one linear combination of $\left\{A^{0}, A^{\alpha}\right\}$ has to be identified with the graviphoton and sits in the gravity multiplet 10 . The corresponding scalar degrees of freedom are provided by $j^{\alpha}, \zeta^{i}, r$ for a total of $\left(n_{T}+1\right)+n_{V}+1$ variables. However, they are subject to one constraint, which in 6d language is given by (3.2). This counting is consistent with the existence of very special coordinates $M^{\mathcal{I}}=\left(M^{0}, M^{\alpha}, M^{i}\right)$ satisfying (3.48).

In the remaining part of this section we discuss in which way, and to which extent, the results of the dimensional reduction performed in 3.2 can be expressed in canonical

\footnotetext{
${ }^{10}$ We include $A^{\alpha}$ because we cannot exclude a contribution from the $6 \mathrm{~d}$ anti-self-dual two-form in the gravity multiplet.
} 
form (3.50). The first step towards this direction is provided by the correct identification of the very special coordinates $M^{\mathcal{I}}$ on the vector multiplet scalar manifold. It turns out that these new coordinates are defined in terms of the old coordinates $\left(r, j^{\alpha}, \zeta^{i}\right)$ by relations

$$
\begin{aligned}
& M^{0}=r^{-4 / 3}, \\
& M^{\alpha}=r^{2 / 3}\left(j^{\alpha}+2 b^{\alpha} r^{-2} C_{i j} \zeta^{i} \zeta^{j}\right), \\
& M^{i}=r^{-4 / 3} \zeta^{i} .
\end{aligned}
$$

Next, let us define

$$
\mathcal{N}^{\mathrm{F}}=\Omega_{\alpha \beta} M^{0} M^{\alpha} M^{\beta}-4 \Omega_{\alpha \beta} b^{\alpha} C_{i j} M^{\beta} M^{i} M^{j}+4 \Omega_{\alpha \beta} b^{\alpha} b^{\beta} C_{i j} C_{k l} \frac{M^{i} M^{j} M^{k} M^{l}}{M^{0}}
$$

Expressions (3.53) and (3.54) are engineered in such a way that

$$
\mathcal{N}^{\mathrm{F}}=\Omega_{\alpha \beta} j^{\alpha} j^{\beta}=1
$$

holds identically. In particular, note that this identity depends on the non-trivial interplay of the non-linear $b^{\alpha}$-shifted redefinition of the coordinates $M^{\alpha}(3.53)$ and the fact that there is a non-polynomial term in the definition (3.54) of $\mathcal{N}^{\mathrm{F}}$, including an inverse power of $M^{0}$. This non-polynomial term in $\mathcal{N}$ is a significant deviation from the canonical case, in which $\mathcal{N}$ is a cubic polynomial, and will be discussed further in the following. However, note that $\mathcal{N}^{\mathrm{F}}$ is still a homogeneous function of degree three in the coordinates $M^{\mathcal{I}}$.

Once the new coordinates $M^{\mathcal{I}}$ are introduced, the $5 \mathrm{~d}$ effective action takes the form

$$
\begin{aligned}
S^{(5) \mathrm{F}}=\int_{\mathcal{M}_{5}} & +\frac{1}{2} R * 1-h_{u v} d q^{u} \wedge * d q^{v}-\frac{1}{2} G_{\mathcal{I} \mathcal{J}} d M^{\mathcal{I}} \wedge * d M^{\mathcal{J}} \\
& -\frac{1}{2} G_{\mathcal{I} \mathcal{J}} F^{\mathcal{I}} \wedge * F^{\mathcal{J}}-\frac{1}{12} X_{\mathcal{I} \mathcal{J} \mathcal{K}} A^{\mathcal{I}} \wedge F^{\mathcal{J}} \wedge F^{\mathcal{K}} .
\end{aligned}
$$

where the metric $G_{\mathcal{I} \mathcal{J}}$ and the coefficients $X_{\mathcal{I} \mathcal{J K}}=X_{\mathcal{I}(\mathcal{J K})}$ are functions of the scalar fields $M^{\mathcal{I}}$. Note that the gauge coupling function and the metric in the kinetic term for scalars $M^{\mathcal{I}}$ coincide, as expected for a $5 \mathrm{~d} \mathcal{N}=2$ theory. Moreover, both $G_{\mathcal{I} \mathcal{J}}$ and $X_{\mathcal{I} \mathcal{J K}}$ are completely determined by the function $\mathcal{N}^{\mathrm{F}}$ introduced above, as explained in the following.

As far as the metric $G_{\mathcal{I} \mathcal{J}}$ is concerned, it is given precisely by (3.49). It is interesting to point out that the non-polynomial term in the definition of $\mathcal{N}^{\mathrm{F}}$ is crucial for (3.49) to hold for the Kaluza-Klein reduced action.

The Chern-Simons term in (3.56),

$$
S_{\mathrm{CS}}^{(5) \mathrm{F}}=-\frac{1}{12} \int_{\mathcal{M}_{5}} X_{\mathcal{I} \mathcal{J K}} A^{\mathcal{I}} \wedge F^{\mathcal{J}} \wedge F^{\mathcal{K}}
$$


deserves more discussion. Its variation under an Abelian gauge transformation $\delta A^{\mathcal{I}}=d \lambda^{\mathcal{I}}$ can be written as a boundary term, plus

$$
\delta S_{\mathrm{CS}}^{(5) \mathrm{F}}=-\frac{1}{12} \int_{\mathcal{M}_{5}} \lambda^{\mathcal{I}} d X_{\mathcal{I} \mathcal{J K}} \wedge F^{\mathcal{J}} \wedge F^{\mathcal{K}} .
$$

For each value of indices $\mathcal{I}, \mathcal{J}, \mathcal{K}$, two possibilities may occur:

1. $X_{\mathcal{I} \mathcal{J K}}$ is constant: the corresponding contribution to the Chern-Simons term is gauge invariant in five dimensions;

2. $X_{\mathcal{I} \mathcal{J K}}$ depends non-trivially on the scalars $M^{\mathcal{I}}$ : the corresponding contribution to the Chern-Simons term breaks 5 d gauge invariance explicitly.

Usually, only the first case is encountered in supergravity models. As a consequence, only the totally symmetric part of $X_{\mathcal{I} \mathcal{J K}}$ effectively enters the action, because we are allowed to integrate by parts and permute indices on the vector and the field strengths in (3.57). This symmetry argument breaks down if some components of $X_{\mathcal{I} \mathcal{J K}}$ are non-constant. In fact, the first slot of this tensor plays a distinguished role: exactly those gauge symmetries are broken, whose gauge vector has index $\mathcal{I}$ such that not all components $\left\{X_{\mathcal{I} \mathcal{J K}}\right\}_{\mathcal{J}, \mathcal{K}}$ are constant, as can be see from (3.58).

As mentioned above, all data needed to construct (3.57) can be extracted from the function $\mathcal{N}^{\mathrm{F}}$ introduced above. To this end, it is useful to note that $\mathcal{N}^{\mathrm{F}}$ naturally splits in a polynomial part $\mathcal{N}_{\mathrm{p}}^{\mathrm{F}}$ and a non-polynomial part $\mathcal{N}_{\mathrm{np}}^{\mathrm{F}}$,

$$
\begin{aligned}
& \mathcal{N}_{\mathrm{p}}^{\mathrm{F}}=\Omega_{\alpha \beta} M^{0} M^{\alpha} M^{\beta}-4 \Omega_{\alpha \beta} b^{\alpha} C_{i j} M^{\beta} M^{i} M^{j} \\
& \mathcal{N}_{\mathrm{np}}^{\mathrm{F}}=4 \Omega_{\alpha \beta} b^{\alpha} b^{\beta} C_{i j} C_{k l} \frac{M^{i} M^{j} M^{k} M^{l}}{M^{0}}
\end{aligned}
$$

On the one hand, since $\mathcal{N}_{\mathrm{p}}^{\mathrm{F}}$ is a homogeneous polynomial of degree three, its third derivatives with respect to coordinates $M^{\mathcal{I}}$ are constants. In fact, they turn out to be simply related to the coefficients of the gauge invariant part of (3.57). On the other hand, third derivatives of $\mathcal{N}_{\mathrm{np}}^{\mathrm{F}}$ are non-constant, and indeed they are proportional to the coefficient functions appearing in the gauge-anomalous contributions to (3.57). More precisely, we have

$$
S_{\mathrm{CS}}^{(5) \mathrm{F}}=-\frac{1}{12} \int_{\mathcal{M}_{5}}\left(\mathcal{N}_{\mathrm{p}}^{\mathrm{F}}\right)_{\mathcal{I} \mathcal{J} \mathcal{K}} A^{\mathcal{I}} \wedge F^{\mathcal{J}} \wedge F^{\mathcal{K}}-\frac{1}{16} \int_{\mathcal{M}_{5}}\left(\mathcal{N}_{\mathrm{np}}^{\mathrm{F}}\right)_{i \mathcal{J K}} A^{i} \wedge F^{\mathcal{J}} \wedge F^{\mathcal{K}}
$$

Two remarks are due at this point. Firstly, observe that the first term fits into the canonical form discussed above, since for a cubic polynomial as (3.47) one has precisely $\mathcal{N}_{\mathcal{I} \mathcal{J K}}=C_{\mathcal{I} \mathcal{J K}}$. Secondly, note that in the second term the first index never takes values $0, \alpha$. This means that the $U(1)$ gauge symmetries associated to vectors $A^{0}, A^{\alpha}$ are unbroken, while those associated to vectors $A^{i}$ are broken.

It may be considered questionable, if not inconsistent, to construct a $5 \mathrm{~d}$ effective action which fails to be gauge invariant. However, this should not come as a surprise. 
Our starting point in six dimensions (3.22) is not gauge invariant as well, because of the introduction of the Green-Schwarz terms. As discussed in section 3.1, these terms are needed in order to implement the anomaly cancellation mechanism: they introduce tree-level gauge violations which counterbalance one-loop anomalous diagrams generated by the chiral matter content of the theory. As a result, the sum of the tree-level and oneloop contributions to the $6 \mathrm{~d}$ effective action is gauge invariant, while the two summands are not invariant separately. This suggests that a gauge invariant $5 \mathrm{~d}$ effective action could be obtained supplementing the computation of this section with the reduction of the one-loop $6 \mathrm{~d}$ effective action. However, we do not need to address this ambitious task, since we will show that all relevant data about the effective action of F-theory in six dimensions can already be extracted from the reduction of the tree-level action only.

It is worth mentioning a crucial distinction between anomalous terms in six and five dimensions. It is well known that $5 \mathrm{~d}$ theories do not develop quantum anomalies. Indeed, possible non-gauge invariant terms can always be cancelled by adding suitable local counter-terms to the tree level action, in such a way that the full effective action at one-loop is gauge-invariant. This kind of anomalies is referred to as 'irrelevant'. The aforementioned counterterms in $5 \mathrm{~d}$ take the form $\int A \wedge * J$, where $A$ is one of the vectors whose gauge invariance is anomalous, and $J$ is a gauge invariant $5 \mathrm{~d}$ current, such that $* J \propto F \wedge F$. It is precisely the gauge invariance of this current which makes the anomaly irrelevant. If we were to implement a similar mechanism to treat $6 \mathrm{~d}$ anomalies, we would have $* J \propto A \wedge F \wedge F$, which is manifestly non gauge invariant.

From this point of view, the non-gauge invariant Chern-Simons term which appears in (3.60) has the same form as the counterterms discussed above. More precisely, the corresponding gauge invariant current reads

$$
* J_{i}=-\frac{1}{16}\left(\mathcal{N}_{\mathrm{np}}^{\mathrm{F}}\right)_{i \mathcal{J K}} F^{\mathcal{I}} \wedge F^{\mathcal{K}} .
$$

Note that all scalar fields in $\left(\mathcal{N}_{\mathrm{np}}^{\mathrm{F}}\right)_{i \mathcal{J K}}$ are neutral under the gauge group $U(1)^{\operatorname{rank}(G)}$ after spontaneous symmetry breaking to the Coulomb branch.

In summary, we are able to cast the Kaluza-Klein reduced action in canonical form, even though some subtle points have to be stressed:

- $\mathcal{N}$ has to be promoted from a cubic polynomial to a homogeneous function $\mathcal{N}^{\mathrm{F}}$ of degree three; the very special geometry constraint $\mathcal{N}^{\mathrm{F}}=1$ and the metric $G_{\mathcal{I} \mathcal{J}}$ are formulated in terms of this non-polynomial $\mathcal{N}^{\mathrm{F}}$;

- the Chern-Simons term coming from Kaluza-Klein reduction and the Chern-Simons term obtained through the canonical prescription $C_{\mathcal{I} \mathcal{J K}}=\left(\mathcal{N}^{\mathrm{F}}\right)_{\mathcal{I} \mathcal{J K}}$ share the same gauge-invariant part, and differ only for non gauge-invariant terms; these can be interpreted as local counterterms which make $5 \mathrm{~d}$ anomalies irrelevant.

Since counterterms are completely specified by the classical data of the model, all information about the effective $5 \mathrm{~d}$ action is encoded in the polynomial part of $\mathcal{N}^{\mathrm{F}}$ and the corresponding gauge-invariant Chern-Simons terms. 


\subsection{Higher order curvature corrections}

As we have seen in subsection 3.1, anomaly cancellation requires the introduction of a higher curvature term in the $6 \mathrm{~d}$ action,

$$
\hat{S}_{\mathcal{R}^{2}}^{(6)}=-\frac{1}{4} \int_{\mathcal{M}_{6}} \Omega_{\alpha \beta} a^{\alpha} \hat{B}^{\beta} \wedge \operatorname{tr} \hat{\mathcal{R}} \wedge \hat{\mathcal{R}}
$$

Furthermore, local Lorentz transformations act non-trivially on the two-forms $\hat{B}^{\alpha}$, in such a way that the corresponding field strength $\hat{G}^{\alpha}$ receives a gravitational contribution. Even if we are not going to perform the dimensional reduction of the complete, higherderivative action, we can make general remarks about some interesting feature of the resulting $5 \mathrm{~d}$ action.

First of all, as stated in subsection 3.2, inclusion of gravitational contributions does not interfere with the possibility to get rid of $5 \mathrm{~d}$ two-forms $B^{\alpha}$ in favour of vectors $A^{\alpha}$. Indeed, gravitational terms modify the action in such a way that $F^{\beta}$ in

$$
\Delta S^{(5) \mathrm{F}}=-\frac{1}{2} \int_{\mathcal{M}_{5}} \Omega_{\alpha \beta} d B^{\alpha} \wedge F^{\beta}
$$

is replaced by a more complicated expression, which is nonetheless exact. $\Delta S^{(5) \mathrm{F}}$ is still a total derivative, and the elimination of $B^{\alpha}$ can proceed along the same line as in the two-derivative case.

Secondly, it can be verified that all possible non-gauge invariant terms in the final $5 \mathrm{~d}$ action are proportional to

$$
\Omega_{\alpha \beta} a^{\alpha} a^{\beta} \quad \text { or } \quad \Omega_{\alpha \beta} a^{\alpha} b^{\beta} \quad \text { or } \quad \Omega_{\alpha \beta} b^{\alpha} b^{\beta} \text {. }
$$

This observation will be relevant for the discussion of F-theory lift, in section 5 .

Finally, let us present one particular higher curvature contribution to the $5 \mathrm{~d}$ action, which will play a prominent role in the matching with M-theory on a Calabi-Yau threefold. It is the $A \mathcal{R} \mathcal{R}$ term coming from dimensional reduction of the $\hat{B} \hat{\mathcal{R}} \hat{\mathcal{R}} 6 \mathrm{~d}$ term written above. In order to extract this term from the total $5 \mathrm{~d}$ action, we can effectively set $A^{0}$ to zero and treat $r$ as a constant:11

$$
\begin{aligned}
& \hat{\mathcal{R}}_{a b}=\mathcal{R}_{a b}+\ldots \\
& \hat{\mathcal{R}}_{a 5}=0+\ldots,
\end{aligned}
$$

where $a, b,=0, \ldots, 4$ are $5 \mathrm{~d}$ flat spacetime indices, and '5' refers to the compact direction. As a consequence, we have

$$
\operatorname{tr} \hat{\mathcal{R}} \wedge \hat{\mathcal{R}}=\operatorname{tr} \mathcal{R} \wedge \mathcal{R}+\ldots
$$

\footnotetext{
${ }^{11}$ The Weyl rescaling $\tilde{g}_{\mu \nu}=r^{-2 / 3} g_{\mu \nu}$ has no effect on the leading, moduli-independent terms in the expression of the curvature two-form.
} 
A first contribution to the term we are looking for is then given by

$$
\frac{1}{4} \int_{\mathcal{M}_{5}} \Omega_{\alpha \beta} a^{\alpha} A^{\beta} \wedge \operatorname{tr} \mathcal{R} \wedge \mathcal{R}
$$

in which the change of sign comes from the Ansatz (3.31). Note however that an addition contribution arises when $\Delta S^{(5) \mathrm{F}}$ is added in order to eliminate tensors from the $5 \mathrm{~d}$ action, as can be seen recalling the definition of $G^{\alpha}(3.35)$ :

$$
-\frac{1}{2} \int_{\mathcal{M}_{5}} \Omega_{\alpha \beta} d B^{\alpha} \wedge F^{\beta} \supset+\frac{1}{4} \int_{\mathcal{M}_{5}} \Omega_{\alpha \beta} a^{\alpha} \omega_{\mathrm{CS}}^{\mathrm{grav}} \wedge F^{\beta}=\frac{1}{4} \int_{\mathcal{M}_{5}} \Omega_{\alpha \beta} a^{\alpha} A^{\beta} \wedge \operatorname{tr} \mathcal{R} \wedge \mathcal{R} .
$$

In summary, we find the $5 \mathrm{~d}$ higher curvature term

$$
S_{A \mathcal{R} R}^{(5) \mathrm{F}}=\frac{1}{2} \int_{\mathcal{M}_{5}} \Omega_{\alpha \beta} a^{\alpha} A^{\beta} \wedge \operatorname{tr} \mathcal{R} \wedge \mathcal{R}
$$

We conclude this subsection describing the effect of higher curvature terms on the canonical form of $5 \mathrm{~d}$ supergravity. As done in [41], superconformal techniques can be used to construct the $5 \mathrm{~d}$ supersymmetric completion of the $A \mathcal{R} \mathcal{R}$ term. In this formalism, the supersymmetry algebra closes off-shell, at the expense of introducing auxiliary fields in the gravity, vector and hypermultiplets. The scalar manifold associated to vector multiplets is still described by constrained coordinates $M^{\mathcal{I}}$. However, the constraint is no longer

$$
\frac{1}{3 !} C_{\mathcal{I} \mathcal{J K}} M^{\mathcal{I}} M^{\mathcal{J}} M^{\mathcal{K}}=1
$$

but gets corrected by terms proportional to the constants $c_{2 \mathcal{I}}$ appearing in front of $A^{\mathcal{I}} \wedge$ $\operatorname{tr} \mathcal{R} \wedge \mathcal{R}$ in the higher derivative Lagrangian [42]:

$$
\frac{1}{3 !} C_{\mathcal{I} \mathcal{J} K} M^{\mathcal{I}} M^{\mathcal{J}} M^{\mathcal{K}}=1-\frac{1}{72} c_{2 \mathcal{I}}\left(D M^{\mathcal{I}}+v^{\mu \nu} F_{\mu \nu}^{\mathcal{I}}\right)
$$

where $D, v_{\mu \nu}$ are the auxiliary bosonic fields in the gravity multiplet. It is possible to integrate them out iteratively in a small $c_{2 \mathcal{I}}$ expansion; the result reads schematically $C M^{3}=1+c F^{2}$.

\section{M-theory on a Calabi-Yau threefold}

In this section we recall the dimensional reduction of M-theory on a Calabi-Yau threefold and adapt it to the case of elliptic fibrations with resolved singularities. The basics of the Kaluza-Klein reduction restricted to the zero-modes are presented in subsection 4.1, with more details summarized in appendix E. We also discuss the the specification to a resolved elliptically fibred Calabi-Yau threefold. In subsection 4.2 we perform the dimensional reduction of a higher curvature correction to 11d supergravity focusing on the terms needed in the matching with the $5 \mathrm{~d}$ higher curvature terms of section 3.5 . 


\subsection{M-theory action on an elliptic Calabi-Yau threefold}

In this subsection, we start with the unique two-derivative action for $11 \mathrm{~d}$ supergravity [43, whose purely bosonic part is

$$
\hat{S}^{(11)}=\int_{\mathcal{M}_{11}} \frac{1}{2} \hat{R} \hat{*} 1-\frac{1}{4} \hat{F}_{4} \wedge \hat{*} \hat{F}_{4}-\frac{1}{12} \hat{C}_{3} \wedge \hat{F}_{4} \wedge \hat{F}_{4}
$$

where $\hat{F}_{4}=d \hat{C}_{3}$. In this subsection a hat will always denote $11 d$ fields. Supergravity compactified from eleven to five dimensions is discussed e.g. in [27].

Following the standard recipe for dimensional reduction on a Calabi-Yau threefold $Y_{3}$, we expand 11d fields on a basis of zero-modes of the appropriate differential operator on the internal manifold. We refer the reader to appendix $\mathrm{D}$ for an account on our notations for Calabi-Yau threefolds. The background metric has a line element

$$
d s_{11}^{2}=\tilde{g}_{\mu \nu}(x) d x^{\mu} d x^{\nu}+2 g_{\bar{\imath} j}(y) d \bar{y}^{\bar{\imath}} d y^{j}
$$

where the external metric $\tilde{g}_{\mu \nu}$ describes a maximally symmetric spacetime (Minkowski, $\mathrm{dS}, \mathrm{AdS}$ ) and a twiddle reminds us that a Weyl rescaling will be performed later. Fluctuations of the internal metric $g_{\bar{\imath} j}$ are zero-modes of the Lichnerowicz operator and are expanded onto the $(1,1)$ and $(1,2)$ cohomologies,

$$
\delta g_{i \bar{\jmath}}=-i\left(\omega_{\Lambda}\right)_{i \bar{\jmath}} \delta v^{\Lambda}, \quad \delta g_{i j}=\left(\bar{b}_{\bar{\kappa}}\right)_{i j} \delta \bar{z}^{\bar{\kappa}}
$$

in which

$$
\left(\bar{b}_{\bar{\kappa}}\right)_{i j}=\frac{i}{\|\Omega\|^{2}}\left(\bar{\chi}_{\bar{\kappa}}\right)_{i \bar{k} \bar{l}} \Omega_{j}^{\bar{k} \bar{l}}
$$

where $\Omega$ is the holomorphic $(3,0)$-form, and $\chi_{\kappa}$ is a basis of $(2,1)$-forms on $Y_{3}$. The variations (4.3) are parameterized by the complex structure moduli $z^{\kappa}$, and the Kähler moduli $v^{\Lambda}$ which are obtained in the expansion of the Kähler form

$$
J=v^{\Lambda} \omega_{\Lambda}
$$

The excitations of the three-form $\hat{C}_{3}$ are zero-modes of the internal Laplacian, and are therefore expanded on a cohomology basis of the internal manifold,

$$
\hat{C}_{3}=\xi^{K} \alpha_{K}-\tilde{\xi}_{K} \beta^{K}+A^{\Lambda} \wedge \omega_{\Lambda}+C_{3}
$$

where $\left(\alpha_{K}, \beta^{K}\right)$ is a real symplectic basis of the middle cohomology of $Y_{3}$. The zero-modes $\left(\xi^{K}, \tilde{\xi}_{K}\right)$ are scalars, $A^{\Lambda}$ are vectors, and $C_{3}$ is a three-form in five dimensions.

Let us now discuss how these fields fit into $5 \mathrm{~d} \mathcal{N}=2$ supersymmetry multiplets. As a preliminary remark, recall that in five dimensions three-forms can be dualized into scalars, so that we are allowed to trade $C_{3}$ for a real scalar field $\Phi$. The gravity multiplet consists of $\tilde{g}_{\mu \nu}$ and of one (linear combination) of the $A^{\Lambda}$ vectors. The remaining vectors fit into

$$
n_{V}^{(5)}=h^{1,1}\left(Y_{3}\right)-1
$$


vector multiplets, along with the Kähler moduli $v^{\Lambda}$. It seems like there is a mismatch of degrees of freedom, since we have $h^{1,1}\left(Y_{3}\right)$ scalars. This seeming difficulty is overcome by the following observation. We introduce the total volume of the Calabi-Yau threefold as

$$
\mathcal{V}=\frac{1}{3 !} \int_{Y_{3}} J \wedge J \wedge J=\frac{1}{3 !} \mathcal{V}_{\Lambda \Sigma \Theta} v^{\Lambda} v^{\Sigma} v^{\Theta}
$$

where $\mathcal{V}_{\Lambda \Sigma \Theta}$ are the intersection numbers of the Calabi-Yau threefold introduced in (2.18). Then, $\mathcal{V}$ actually sits in the universal hypermultiplet, leaving effectively $h^{1,1}\left(Y_{3}\right)-1$ scalar degrees of freedom in the vector sector.

To discuss hypermultiplets we need to recall the decomposition of the third cohomology into complex cohomologies,

$$
H^{3}\left(Y_{3}\right)=\left[H^{1,2}\left(Y_{3}\right) \oplus H^{2,1}\left(Y_{3}\right)\right] \oplus\left[H^{0,3}\left(Y_{3}\right) \oplus H^{3,0}\left(Y_{3}\right)\right] .
$$

Real scalars $\xi^{K}, \tilde{\xi}_{K}$ provide $h^{1,2}\left(Y_{3}\right)+1$ complex degrees of freedom: $h^{1,2}\left(Y_{3}\right)$ of these correspond to the $H^{1,2}\left(Y_{3}\right) \oplus H^{2,1}\left(Y_{3}\right)$ component and combine with the complex structure moduli $z^{\kappa}$ to give $h^{1,2}\left(Y_{3}\right)$ hypermultiplets; the remaining complex degree of freedom lives in $H^{0,3}\left(Y_{3}\right) \oplus H^{3,0}\left(Y_{3}\right)$ and combines with $\mathcal{V}, \Phi$ in the universal hypermultiplet. In conclusion, we have found

$$
n_{H}^{(5)}=h^{1,2}\left(Y_{3}\right)+1
$$

hypermultiplets, which will be collectively denoted by $q^{u}$.

The dimensional reduction is carried out in detail in appendix E. Since the overall volume sits in the universal hypermultiplet it is natural to define scalar fields

$$
L^{\Lambda}=\mathcal{V}^{-\frac{1}{3}} v^{\Lambda}
$$

which are the real scalars in the vector multiplets. They only parameterize $h^{1,1}\left(Y_{3}\right)-1$ degrees of freedom, since due to their definition they are subject to the constraint

$$
\frac{1}{3 !} \mathcal{V}_{\Lambda \Sigma \Theta} L^{\Lambda} L^{\Sigma} L^{\Theta}=1
$$

We are naturally led to interpret $L^{\Lambda}$ as 5 d very special coordinates, in term of which the cubic potential reads

$$
\mathcal{N}=\frac{1}{3 !} \mathcal{V}_{\Lambda \Sigma \Theta} L^{\Lambda} L^{\Sigma} L^{\Theta}
$$

Some additional details of this $5 \mathrm{~d}$ formalism have been already given in section 3.4 .

Once the cubic potential $\mathcal{N}$ is known, the only missing ingredient to specify the model is the quaternionic metric $h_{u v}$ on the hypermultiplet scalar manifold: its expression in terms of $\mathcal{V}, \Phi, \xi^{K}, \tilde{\xi}_{K}, z^{\kappa}$ can be found in appendix E. In summary, the reduced bosonic action reads

$$
\begin{aligned}
S^{(5) \mathrm{M}}=\int_{\mathcal{M}_{5}} & +\frac{1}{2} R * 1-\frac{1}{2} G_{\Lambda \Sigma} d L^{\Lambda} \wedge * d L^{\Sigma}-h_{u v} d q^{u} \wedge * d q^{v} \\
& -\frac{1}{2} G_{\Lambda \Sigma} F^{\Lambda} \wedge * F^{\Sigma}-\frac{1}{12} \mathcal{V}_{\Lambda \Sigma \Theta} A^{\Lambda} \wedge F^{\Sigma} \wedge F^{\Theta}
\end{aligned}
$$


where, as expected,

$$
G_{\Lambda \Sigma}=\left[-\frac{1}{2} \partial_{L^{\Lambda}} \partial_{L^{\Sigma}} \log \mathcal{N}\right]_{\mathcal{N}=1}
$$

When restricted to elliptic fibrations as discussed next, it will be this form of the $5 \mathrm{~d}$ action which can be matched to the circle reduced action of section 3.4.

Let us now specify this result to the elliptically fibred geometry introduced in subsection 2.2 . We first split the index $\Lambda$ into $(0, \alpha, i)$ and write

$$
L^{\Lambda}=\left(R, L^{\alpha}, \xi^{i}\right), \quad A^{\Lambda}=\left(A^{0}, A^{\alpha}, A^{i}\right)
$$

Combining this notation with the intersection numbers (2.19) of an elliptic fibration we get

$$
\begin{aligned}
\mathcal{N}= & \frac{1}{2} \eta_{\alpha \beta} R L^{\alpha} L^{\beta}+\frac{1}{2} \eta_{\alpha \beta} K^{\alpha} R^{2} L^{\beta}+\frac{1}{6} \eta_{\alpha \beta} K^{\alpha} K^{\beta} R^{3} \\
& -\frac{1}{2} \eta_{\alpha \beta} C^{\alpha} C_{i j} L^{\beta} \xi^{i} \xi^{j}+\frac{1}{6} \mathcal{V}_{i j k} \xi^{i} \xi^{j} \xi^{k}
\end{aligned}
$$

As we will discuss in section 5 couplings of the form $R^{2} L^{\alpha}$ in (4.17) are not compatible with the $6 \mathrm{~d} / 5 \mathrm{~d}$ lift. However, there is as simple field redefinition which allows us to get rid of these $R^{2} L^{\alpha}$ terms. More precisely, one introduces the shifted fields 12

$$
\check{L}^{\alpha}=L^{\alpha}+\frac{1}{2} K^{\alpha} R, \quad \check{A}^{\alpha}=A^{\alpha}+\frac{1}{2} K^{\alpha} A^{0}
$$

where the shift of the vectors is required by supersymmetry. Clearly, the new $\check{L}^{\alpha}$, and new vectors can be obtained by expanding $J$ and $C_{3}$ in a new basis of two-forms

$$
\check{\omega}_{0}=\omega_{0}-\frac{1}{2} K^{\alpha} \omega_{\alpha}, \quad \check{\omega}_{\alpha}=\omega_{\alpha}, \quad \check{\omega}_{i}=\omega_{i} .
$$

In fact, this new basis is better suited to identify the vectors $\check{A}^{\alpha}$ as dualizable into $5 \mathrm{~d}$ tensors. The cubic potential in the new coordinates given by

$$
\begin{aligned}
\mathcal{N}^{\mathrm{M}}= & \frac{1}{2} \eta_{\alpha \beta} R \check{L}^{\alpha} \check{L}^{\beta}+\frac{1}{24} \eta_{\alpha \beta} K^{\alpha} K^{\beta} R^{3} \\
& -\frac{1}{2} \eta_{\alpha \beta} C^{\alpha} C_{i j} \check{L}^{\beta} \xi^{i} \xi^{j}+\frac{1}{4} \eta_{\alpha \beta} C^{\alpha} C_{i j} K^{\beta} R \xi^{i} \xi^{j}+\frac{1}{6} \mathcal{V}_{i j k} \xi^{i} \xi^{j} \xi^{k}
\end{aligned}
$$

Using this expression of $\mathcal{N}$ the Chern-Simons term takes the form

$$
\begin{aligned}
S_{\mathrm{CS}}^{(5) \mathrm{M}}=\int_{\mathcal{M}_{5}} & -\frac{1}{4} \eta_{\alpha \beta} A^{0} \wedge \check{F}^{\alpha} \wedge \check{F}^{\beta}+\frac{1}{4} \eta_{\alpha \beta} C^{\alpha} C_{i j} \check{A}^{\alpha} \wedge F^{i} \wedge F^{j} \\
& -\frac{1}{48} \eta_{\alpha \beta} K^{\alpha} K^{\beta} A^{0} \wedge F^{0} \wedge F^{0}-\frac{1}{8} \eta_{\alpha \beta} C^{\alpha} C_{i j} K^{\beta} A^{0} \wedge F^{i} \wedge F^{j} \\
& -\frac{1}{12} \mathcal{V}_{i j k} A^{i} \wedge F^{j} \wedge F^{k},
\end{aligned}
$$

where $\check{F}^{\alpha}$ is the usual field strength of the vectors $\check{A}^{\alpha}$ introduced in (4.18).

\footnotetext{
${ }^{12}$ This field redefinition is also crucial in the $4 \mathrm{~d} / 3 \mathrm{~d}$ treatment of F-theory on Calabi-Yau fourfolds as discussed in [44, 25].
} 


\subsection{Higher order curvature corrections}

Several higher-derivative corrections to the 11d M-theory action (4.1) are known [45, 46]. In the following, we will focus on the mixed gauge-gravitational correction 13

$$
\hat{S}_{C \mathcal{R}^{4}}^{(11)}=\frac{1}{96} \int_{\mathcal{M}_{11}} \hat{C}_{3} \wedge\left[\operatorname{tr} \hat{\mathcal{R}}^{4}-\frac{1}{4}\left(\operatorname{tr} \hat{\mathcal{R}}^{2}\right)^{2}\right]
$$

because this terms allows us to make direct contact to the higher-derivative corrections studied in subsection 3.5.

Rather than performing a complete dimensional reduction of (4.22), we will extract the relevant terms and we will systematically neglect all contributions which involve gradients of the Kähler and complex structure moduli. This means that we can effectively neglect fluctuations and compute curvature invariants on the background, which is the product space $\mathcal{M}_{11}=\mathcal{M}_{5} \times Y_{3}$. As a result, we have simply 14

$$
\hat{\mathcal{R}}=\mathcal{R}+\mathcal{R}_{Y_{3}}
$$

where $\mathcal{R}_{Y_{3}}$ is the curvature two-form on the Calabi-Yau threefold, and $\mathcal{R}$ is the $5 \mathrm{~d}$ curvature two-form. A straightforward computation gives then

$$
\left(\operatorname{tr} \hat{\mathcal{R}}^{2}\right)^{2}=2 \operatorname{tr} \mathcal{R}^{2} \wedge \operatorname{tr} \mathcal{R}_{Y_{3}}^{2}+\ldots, \quad \operatorname{tr} \hat{\mathcal{R}}^{4}=0+\ldots ;,
$$

where the dots are a reminder of the moduli-dependent, neglected terms. It is useful to recall the definition of the first Pontryagin class of the Calabi-Yau threefold $Y_{3}$,

$$
p_{1}\left(Y_{3}\right)=-\frac{1}{2} \operatorname{tr} \mathcal{R}_{Y_{3}}^{2},
$$

and its relation with the second Chern class,

$$
p_{1}\left(Y_{3}\right)=-2 c_{2}\left(Y_{3}\right)
$$

Combining these equations with the three-form expansion (4.6), we can deduce that the $11 \mathrm{~d}$ correction (4.22) yields, among other terms, the following $5 \mathrm{~d}$ correction [28]

$$
S_{A \mathcal{R} \mathcal{R}}^{(5) \mathrm{M}}=\frac{1}{48} \check{c}_{\Lambda} \int_{\mathcal{M}_{5}} \check{A}^{\Lambda} \wedge \operatorname{tr} \mathcal{R}^{2},
$$

where we have defined

$$
\check{c}_{\Lambda}=\int_{Y_{3}} \check{\omega}_{\Lambda} \wedge c_{2}\left(Y_{3}\right) .
$$

\footnotetext{
${ }^{13}$ As discussed in section 3.1. factors of $(2 \pi)^{-1}$ are understood in $\hat{\mathcal{R}}$. Moreover, the relative normalization of this higher-derivative term and the two-derivative action (4.1) depends on the value of the $11 \mathrm{~d}$ gravitational constant. It is suppressed everywhere, adopting a convention which is best suited to make contact with the $6 \mathrm{~d}$ Green-Schwarz term, in which the $6 \mathrm{~d}$ gravitational constant has been equally suppressed.

${ }^{14}$ Just like in the reduction from six to five dimensions, performing the Weyl rescaling on the $5 \mathrm{~d}$ metric does not affect the moduli-independent terms in the expression of the curvature two-form.
} 
To make further progress it is crucial to specialize to the case of an elliptically fibred Calabi-Yau threefold $Y_{3}$. Let us discuss a smooth fibration first. The second Chern class of the total space can then be expressed in term of Chern classes on the base space $B_{2}$, by means of [48]

$$
c_{2}\left(Y_{3}\right)=c_{2}\left(B_{2}\right)+11 c_{1}^{2}\left(B_{2}\right)+12 \omega_{0} \wedge c_{1}\left(B_{2}\right) .
$$

Making use of (2.12) we get

$$
\int_{Y_{3}} \omega_{0} \wedge c_{2}\left(Y_{3}\right)=\int_{Y_{3}} \omega_{0} \wedge\left[c_{2}\left(B_{2}\right)-c_{1}^{2}\left(B_{2}\right)\right]=\int_{B_{2}} c_{2}\left(B_{2}\right)-c_{1}^{2}\left(B_{2}\right) .
$$

This equation can be evaluated further by using the explicit expressions of the integrals of $c_{2}$ and $c_{1}^{2}$ on $B_{2}$ given in (2.14) as

$$
\int_{Y_{3}} \omega_{0} \wedge c_{2}\left(Y_{3}\right)=2 h^{1,1}\left(B_{2}\right)-8 \text {. }
$$

Furthermore, we can also evaluate the second Chern class on the basis $\omega_{\alpha}$ as

$$
\int_{Y_{3}} \omega_{\alpha} \wedge c_{2}\left(Y_{3}\right)=\int_{Y_{3}} \omega_{\alpha} \wedge\left[c_{2}\left(B_{2}\right)+11 c_{1}^{2}\left(B_{2}\right)+12 \omega_{0} \wedge c_{1}\left(B_{2}\right)\right] .
$$

Since the first two terms have all their indices on the base, only the last term provides a non-vanishing contribution. Using $c_{1}\left(B_{2}\right)=-K^{\alpha} \omega_{\alpha}$, as introduced in subsection [2.2, we compute

$$
\check{c}_{\alpha}=\int_{Y_{3}} \check{\omega}_{\alpha} \wedge c_{2}\left(Y_{3}\right)=-12 \eta_{\alpha \beta} K^{\beta},
$$

where we have used $\check{\omega}_{\alpha}=\omega_{\alpha}$. In order to obtain $\check{c}_{0}$ from (4.31), (4.32) we have to recall the definition (4.19) of $\check{\omega}_{0}$, and find

$$
\check{c}_{0}=52-4 h^{1,1}\left(B_{2}\right) .
$$

So far we have worked on a smooth elliptic fibration. We now include the effects of singularities and their resolution. Clearly, the presence of resolved singularities induces new couplings

$$
\check{c}_{i}=\int_{\tilde{Y}_{3}} \check{\omega}_{i} \wedge c_{2}\left(Y_{3}\right)
$$

One expects that this expression evaluated for a given gauge group has a group theoretic interpretation. Giving its precise form is beyond the scope of this work. However, let us note that also the other couplings $\check{c}_{0}$ and $\check{c}_{\alpha}$ could be corrected by the inclusion of blow-up divisors. Indeed, a general shift of $c_{2}\left(\tilde{Y}_{3}\right)$ with the blow-up divisors induces

$$
\int_{\tilde{Y}_{3}} \omega_{0} \wedge \Delta c_{2}\left(\tilde{Y}_{3}\right)=0, \quad \int_{\tilde{Y}_{3}} \omega_{\alpha} \wedge \Delta c_{2}\left(\tilde{Y}_{3}\right)=\mathcal{C}^{i j} \int_{\tilde{Y}_{3}} \omega_{\alpha} \wedge \omega_{i} \wedge \omega_{j},
$$

where we have used the vanishing of the intersections (2.19) with only one $\omega_{i}$ and two $\omega_{\alpha}$, and $\omega_{i} \wedge \omega_{0}=0$. Note that a shift in $\check{c}_{0}$ could still be induced due to the basis change 
(4.19) inducing a term proportional to $\check{c}_{\alpha}$. We claim that also $\check{c}_{\alpha}$ is uncorrected, and thus $\check{c}_{0}$ and $\check{c}_{\alpha}$ remain unchanged. Despite that we do not have a general proof, we have checked for many examples that (4.33) and (4.34) are still true:

$$
\check{c}_{\alpha}=-12 \eta_{\alpha \beta} K^{\beta}, \quad \check{c}_{0}=52-4 h^{1,1}\left(B_{2}\right) .
$$

As we will show later, the fact that $\check{c}_{\alpha}$ is not changed is consistent with the F-theory lift. The fact that $\check{c}_{0}$ does not change in this case follows from (4.36).

\section{$5 \quad$ F-theory lift and one-loop corrections}

In this section we compare the result of the circle reduction of the general $6 \mathrm{~d}(1,0)$ supergravity theory with the M-theory reduction on an elliptically fibred Calabi-Yau threefold. We identify terms which appear at classical level on both sides and can be immediately matched as discussed in subsection 5.1. We also comment on the matching of certain higher derivative terms. It is crucial insight that both reductions contain additional terms which have no immediate analogue in the dual reduction. We suggest in subsection 5.2 that these terms arise at the quantum level and encode the same information about the underlying fully quantized theory. In particular, we argue that certain intersections on the M-theory side correspond in the $6 \mathrm{~d} / 5 \mathrm{~d}$ reduction on a circle to one-loop corrections with charged matter fermions and Kaluza-Klein modes of all $6 \mathrm{~d}$ chiral fields running in the loop. In conclusion this allows us to extract all data from M-theory required to specify the $6 \mathrm{~d}$ action including the complete information about $6 \mathrm{~d}$ anomalies.

\subsection{Classical action in the F-theory lift}

In order to extract information about F-theory in six dimensions, we have to compare the $5 \mathrm{~d}$ action coming from Kaluza-Klein reduction from six dimensions with the $5 \mathrm{~d}$ action of M-theory on an elliptically fibred Calabi-Yau threefold. Our strategy will be similar to the $4 \mathrm{~d} / 3 \mathrm{~d}$ treatment of F-theory on Calabi-Yau fourfolds presented in [25].

As a first step, we present the match of the number of multiplets in five dimensions in order to give the number of $6 \mathrm{~d}$ multiplets in terms of the topological data of the Ftheory compactification manifold $Y_{3}$. This was already implicit in our choice of indices in sections 3 and 4 . More precisely, for the $\alpha$-index we find that the number of $6 \mathrm{~d}$ tensors is given by

$$
n_{T}+1=h^{1,1}\left(B_{2}\right),
$$

where we recall that there are $n_{T} 6 \mathrm{~d}$ tensor multiplets and 1 tensor in the gravity multiplet. In the F-theory reduction the tensors arise from the reduction of the Type IIB RR four-form into a base of $H^{2}\left(B_{2}\right)$. Since $A^{i}$ parameterize the Coulomb branch of the $6 \mathrm{~d} / 5 \mathrm{~d}$ gauge theory, one finds

$$
\operatorname{rank}(G)=h^{1,1}\left(\tilde{Y}_{3}\right)-h^{1,1}\left(B_{2}\right)-1
$$


which counts the number of independent blow-up divisors induced to resolve the singular elliptic fibration to obtain $\tilde{Y}_{3}$. Note that for ADE gauge groups $G$ the number of $6 \mathrm{~d}$ vector multiplets is then given by

$$
n_{V}=\left(c_{G}+1\right) \operatorname{rank}(G),
$$

where $c_{G}$ is the dual Coxeter number of $G$. In F-theory these vectors arise from the seven-brane gauge potentials. Finally, one can match the number of hypermultiplets, simply by noting that a $6 \mathrm{~d}$ hypermultiplet becomes a $5 \mathrm{~d}$ hypermultiplet in the circle reduction. This leads to the following number of neutral $6 \mathrm{~d}$ multiplets

$$
n_{H}^{\text {neutral }}=h^{2,1}\left(\tilde{Y}_{3}\right)+1 .
$$

In F-theory on $Y_{3}$ these neutral hypermultiplets contain the complex deformations of the seven-branes and their Wilson line moduli 15 The universal hypermultiplet in the Ftheory reduction contains as one complex scalar the volume of the base together with the scalar of the Type IIB RR four-form expanded in the volume form of $B_{2}$. The remaining two real scalar degrees of freedom in the universal hypermultiplet arise in the expansion of the Type IIB RR and NSNS two-forms into the universal two-form mode present for any $B_{2}$. The proof of the match (5.1)-(5.4) follows from the match of the effective theories presented in the following.

In order to systematically approach the match of the effective action, we would first like to identify the terms which are classical on both sides. This is not hard for the $6 \mathrm{~d} / 5 \mathrm{~d}$ reduction. More complicated is the distinction of the various terms in the Mtheory potential. We will address the two sides in turn.

In the $6 \mathrm{~d} / 5 \mathrm{~d}$ reduction performed in section 3 we found that there is a potential $\mathcal{N}^{\mathrm{F}}$ given in (3.54) which encodes the kinetic terms of the gauge coupling functions and the Chern-Simons terms in the $5 \mathrm{~d}$ reduced action. It is crucial to recall the natural decomposition of $\mathcal{N}^{\mathrm{F}}$ in (3.54) into a polynomial and a non-polynomial part:

$$
\begin{aligned}
& \mathcal{N}_{\mathrm{p}}^{\mathrm{F}}=\Omega_{\alpha \beta} M^{0} M^{\alpha} M^{\beta}-4 \Omega_{\alpha \beta} b^{\alpha} C_{i j} M^{\beta} M^{i} M^{j}, \\
& \mathcal{N}_{\mathrm{np}}^{\mathrm{F}}=4 \Omega_{\alpha \beta} b^{\alpha} b^{\beta} C_{i j} C_{k l} \frac{M^{i} M^{j} M^{k} M^{l}}{M^{0}}
\end{aligned}
$$

The terms in $\mathcal{N}_{\mathrm{p}}^{\mathrm{F}}$ are cubic and hence encode a standard $\mathcal{N}=25 \mathrm{~d}$ action. In contrast $\mathcal{N}_{\mathrm{np}}^{\mathrm{F}}$ is only homogeneous of degree three, but non-polynomial. As argued in section 3.4 it can be interpreted as a counterterm of the $5 \mathrm{~d}$ one-loop effective action. Its $6 \mathrm{~d}$ origin is related to the classical lack of gauge invariance of the $6 \mathrm{~d}$ action. In fact, it vanishes precisely when

$$
\Omega_{\alpha \beta} b^{\alpha} b^{\beta}=0 .
$$

This corresponds to the case where the $6 \mathrm{~d}$ action is gauge invariant as inferred from (3.24), and is consistent with the absence of $6 \mathrm{~d}$ anomalies as discussed in appendix B,

\footnotetext{
${ }^{15}$ See ref. [4], for a detailed matching with the orientifold picture with D7-branes.
} 
Let us now turn to the M-theory reduction. Here the identification of the classical terms is more subtle. We have worked on the resolved space with finite size elliptic fibre. As discussed in the introductory section 2, the F-theory limit corresponds to both shrinking the blow-up divisors as well as the size of the elliptic fibre. One expects that this selects classical terms in the potential $\mathcal{N}^{\mathrm{M}}$ of equation (4.20). It turns out to be useful to introduce an $\epsilon$-scaling to distinguish various terms in $\mathcal{N}^{\mathrm{M}}$. For the volumes $v^{0}, v^{\alpha}, v^{i}$ appearing in the Kähler form $J=v^{\Lambda} \omega_{\Lambda}$, we make the formal replacements

$$
v^{0} \mapsto \epsilon v^{0}, \quad v^{\alpha} \mapsto \epsilon^{-1 / 2} v^{\alpha}, \quad v^{i} \mapsto \epsilon^{1 / 4} v^{i}
$$

Note that these scalings satisfy some important consistency checks. Firstly, the size of the elliptic fibre $v^{0}$ and the blow-up fibres $v^{i}$ vanish for $\epsilon \rightarrow 0$. Secondly, the total volume $\mathcal{V}$ of $Y_{3}$ is finite, which is required by the fact that $\mathcal{V}$ sits in a 5 d hypermultiplet. Translated into the variables $R, L^{\alpha}, \xi^{i}$ one finds the replacements

$$
R \mapsto \epsilon R, \quad L^{\alpha} \mapsto \epsilon^{-1 / 2} L^{\alpha}, \quad \xi^{i} \mapsto \epsilon^{1 / 4} \xi^{i} .
$$

Since the redefined scalars $\check{L}^{\alpha}$ contain $L^{\alpha}$ linearly, they obey the same rescaling as $L^{\alpha}$. In the limit $\epsilon \rightarrow 0$ two terms in (4.20) survive which we collect in $\mathcal{N}_{\text {class }}^{\mathrm{M}}$. We thus divide the terms in (4.20) into

$$
\begin{aligned}
& \mathcal{N}_{\text {class }}^{\mathrm{M}}=\frac{1}{2} \eta_{\alpha \beta} R \check{L}^{\alpha} \check{L}^{\beta}-\frac{1}{2} \eta_{\alpha \beta} C^{\alpha} C_{i j} \check{L}^{\beta} \xi^{i} \xi^{j} \\
& \mathcal{N}_{\text {loop }}^{\mathrm{M}}=\frac{1}{24} \eta_{\alpha \beta} K^{\alpha} K^{\beta} R^{3}+\frac{1}{4} \eta_{\alpha \beta} C^{\alpha} C_{i j} K^{\beta} R \xi^{i} \xi^{j}+\frac{1}{6} \mathcal{V}_{i j k} \xi^{i} \xi^{j} \xi^{k}
\end{aligned}
$$

It is now straightforward to match $\mathcal{N}_{\text {class }}^{\mathrm{M}}$ with $\mathcal{N}_{\mathrm{p}}^{\mathrm{F}}$ given in $(5.5)$. Note that the second term $\mathcal{N}_{\text {loop }}^{\mathrm{M}}$ in (5.9) will be later reinterpreted as a loop correction, which gives another justification of the split induced by the F-theory limit (5.8).

Let us first start by matching the fields on the $6 \mathrm{~d} / 5 \mathrm{~d}$ and the M-theory side. In order to do that we have to fix the normalization of the fields, which cannot be uniquely extracted by comparing (5.5) and (5.9). Supersymmetry relates the normalization of the real scalars and vectors in the vector multiplets. Hence, given a fixed normalization of the vectors the complete match of the scalar components can be inferred. On the one hand, in the $6 \mathrm{~d} / 5 \mathrm{~d}$ compactification the vectors are normalized by the Green-Schwarz term (3.16), and the fixed definition of the anomaly coefficients $b^{\alpha}, a^{\alpha}$. On the other hand, in M-theory the normalization of the vectors is fixed by a choice of integral basis in the expansion (4.6) of $\hat{C}_{3}$. Appropriately rescaling the $6 \mathrm{~d}$ vectors to also adopt to an integral basis, one can infer the map

$$
M^{0}=2 R, \quad M^{\alpha}=\frac{1}{2} \check{L}^{\alpha}, \quad M^{i}=\frac{1}{2} \xi^{i},
$$

while the constants are identified as

$$
\Omega_{\alpha \beta}=\eta_{\alpha \beta}, \quad b^{\alpha}=C^{\alpha} .
$$

Note that our result are consistent with the findings of [18, 19]. 
So far we have only discussed the vector and gravity sectors of the M-theory to Ftheory matching. Clearly, both the $6 \mathrm{~d} / 5 \mathrm{~d}$ reduction as well as the M-theory reduction contain a hypermultiplet sector. As discussed in section 3.3, we found that in the dimensional reduction from six to five dimensions the charged hypermultiplets are massive in the Coulomb branch. Therefore, they are not visible in the effective action of the massless modes of M-theory. We will include them in the study of loop corrections in the next subsections. However, the neutral hypermultiplets are massless and their moduli space could be matched straightforwardly also leading to (5.4).

Let us close this subsection by also comparing the classical parts of the higher curvature terms dimensionally reduced in sections 3.5 and 4.2 . We have focussed on the terms involving the $5 \mathrm{~d}$ vectors and two $5 \mathrm{~d}$ curvature forms $\mathcal{R}$. In (3.66) and (4.27) we found that such couplings are given by

$$
S_{A \mathcal{R} \mathcal{R}}^{(5) \mathrm{F}}=-\frac{1}{2} \Omega_{\alpha \beta} a^{\beta} \int_{\mathcal{M}_{5}} A^{\alpha} \wedge \operatorname{tr} \mathcal{R}^{2}, \quad S_{A \mathcal{R} \mathcal{R}}^{(5) \mathrm{M}}=\frac{1}{48} \check{c}_{\Lambda} \int_{\mathcal{M}_{5}} \check{A}^{\Lambda} \wedge \operatorname{tr} \mathcal{R}^{2} .
$$

Recall that the coefficients $\check{c}_{\Lambda}$ have been determined in (4.37), and (4.35). Since in the $6 \mathrm{~d} / 5 \mathrm{~d}$ reduction only the $A^{\alpha}$ appears, one suspects that, similar to the F-theory limit discussed above, that these are the only classical terms in the reduction. Using $\check{c}_{\alpha}=-12 \eta_{\alpha \beta} K^{\beta}$, as given in (4.37), we can apply the identification (5.11) to infer

$$
a^{\alpha}=K^{\alpha}
$$

Note that this is precisely, the identification dictated by anomaly cancellation conditions as found in [18, 19]. On the M-theory side we also found the non-vanishing couplings involving $\check{c}_{i}, \check{c}_{0}$. Similar to the split found for $\mathcal{N}^{\mathrm{M}}$ we believe that these couplings are induced by one-loop corrections on the F-theory side. The remainder of this paper is devoted to the discussion of such one-loop quantum corrections.

\subsection{Completing the duality using one-loop corrections}

As we have seen in the previous subsection, only some terms of the $5 \mathrm{~d}$ cubic potential $\mathcal{N}^{\mathrm{M}}$ of M-theory compactified on a Calabi-Yau threefold admit a straightforward dual in the potential $\mathcal{N}^{\mathrm{F}}$ arising from circle compactification of $6 \mathrm{~d}$ supergravity. In this subsection, we will provide a framework for the interpretation of the remaining terms in $\mathcal{N}^{\mathrm{M}}$, which we record here again for the ease of the reader,

$$
\mathcal{N}_{\text {loop }}^{\mathrm{M}}=\frac{1}{24} \eta_{\alpha \beta} K^{\alpha} K^{\beta} R^{3}+\frac{1}{4} \eta_{\alpha \beta} C^{\alpha} C_{i j} K^{\beta} R \xi^{i} \xi^{j}+\frac{1}{6} \mathcal{V}_{i j k} \xi^{i} \xi^{j} \xi^{k}
$$

Recall that $5 \mathrm{~d} \mathcal{N}=2$ supersymmetry ensures that exactly the same amount of information is contained in the cubic potential $\mathcal{N}$ and in the Chern-Simons couplings of vectors. The following discussion is conveniently formulated in terms of the latter. As already anticipated, we relate these couplings to one-loop effects in the $6 \mathrm{~d} / 5 \mathrm{~d}$ dual description. 
In order to clarify the precise meaning of this statement, let us analyse in more detail the origin of Chern-Simons couplings in the effective 5d theory arising from $6 \mathrm{~d}$ supergravity on a circle. A possible source of this kind of interactions is of course provided by dimensional reduction of the Green-Schwarz term in the classical 6d action. These interactions are precisely the ones which we have considered in the previous subsection. However, additional contributions arise, which are understood in the framework of effective quantum field theory. In fact, from a quantum perspective, the $5 \mathrm{~d}$ effective action resulting from compactification on a circle of $6 \mathrm{~d}$ supergravity encodes all information about the low-energy dynamics, including interactions induced by massive fields which have to be integrated out when we restrict our attention to the lightest states of the theory.

In the case under examination, we identify two different families of massive fields which can alter 5 d effective couplings:

- Kaluza-Klein modes. All 6d fields can be schematically expanded into Kaluza-Klein modes as

$$
\hat{\varphi}(x, y)=\sum_{n \in \mathbb{Z}} \varphi^{(n)}(x) e^{i n y}
$$

The modes $\varphi^{(n)}$ with non-zero $n$ appear in the 5 d theory as massive fields, with mass inversely proportional to the radius $r$ of the compactification circle $16, m^{(n)} \sim|n| / r$. As argued in the introductory section 2.1, zero-modes only are sufficient to fix all data needed to specify the $6 \mathrm{~d}$ model we are compactifying, and this is why we have systematically neglected excited modes so far. Nonetheless, Kaluza-Klein modes can run in 5 d loop diagrams.

- Fields which are given a mass by gauge symmetry breaking. Recall that F-/M-theory duality can be applied in a geometric regime only if the 5 d gauge symmetry is spontaneously broken down to the Coulomb phase and the compactification threefold is resolved. This amounts to giving non-vanishing VEVs to some scalars in the vector multiplets. As described in subsection 3.3, these VEVs provide mass terms for the W-bosons and the scalars in charged hypermultiplets. Supersymmetry implies that their fermionic partners, gaugini and hyperini, get massive as well. We claim that these fields can run in $5 \mathrm{~d}$ loops in such a way as to induce effective Chern-Simons couplings.

We are able to provide a geometric picture for these families in the F-theory set-up. As recalled in the previous subsection, F-theory is conveniently analysed in a phase with finite size of the elliptic fibre and of the exceptional divisors introduced by resolution of singularities. However, F-/M-theory duality holds only in the limit in which these cycles are shrunk to zero size. In the M-theory picture, M2-branes can wrap these shrinking submanifolds. By means of the chain of dualities described in section 2.1, it is possible to identify the states of M2-branes wrapping the elliptic fibre as Kaluza-Klein modes

\footnotetext{
${ }^{16}$ This holds before possible Weyl rescalings are taken into account.
} 
in the $6 \mathrm{~d} / 5 \mathrm{~d}$ picture. Furthermore, $5 \mathrm{~d}$ Higgsing to the Coulomb branch is dual to the blowing-up of singularities provided by exceptional divisors. M2-branes wrapping such divisors provide the degrees of freedom of both $\mathrm{W}$-bosons and charged hypermultiplets, whose mass vanishes as the divisor is blown-down.

We now turn to a more detailed description of the mechanism responsible for ChernSimons couplings in the effective $5 \mathrm{~d}$ theory. We follow closely reference 30]. A term of the form

$$
A \wedge F \wedge F
$$

in the Lagrangian corresponds to an amplitude with three external vectors. If these carry momenta $p, q,-p-q$ and polarizations $\alpha, \beta, \gamma$, the amplitude will be proportional to

$$
\epsilon^{\alpha \beta \gamma \mu \nu} p_{\mu} q_{\nu} .
$$

Suppose we compute a three-vector amplitude in the $5 \mathrm{~d}$ theory with massive fields of the kind listed above. General arguments imply that only one-loop diagrams provide corrections to the classical Chern-Simons interactions. It is crucial to observe that the structure of the Chern-Simons coupling we are interested in can be extracted unambiguously by looking at the parity violating terms with quadratic dependence on the external momenta $p, q$. In particular, a Chern-Simons effective coupling can arise only if a totally antisymmetric tensor $\epsilon^{\alpha \beta \gamma \mu \nu}$ is found in the computation of the three-vector amplitude.

We argue that this tensorial structure can be generated if massive modes of $6 \mathrm{~d}$ chiral fields run in the loop. First of all, vertices between fermions and vectors are able to give this kind of parity violating term. From a Feynman diagram perspective, this can be seen as follows. In the computation of a one-loop amplitude with fermions running in the loop, the trace of a string of $5 \mathrm{~d}$ gamma matrices is involved. However, $5 \mathrm{~d}$ Clifford algebra implies, e.g.

$$
\begin{aligned}
\operatorname{tr} \Gamma_{a} \Gamma_{b} \Gamma_{c} \Gamma_{d} \Gamma_{e} & =4 \epsilon_{a b c d e}, \\
\operatorname{tr} \Gamma_{a} \Gamma_{b} \Gamma_{c} \Gamma_{d} \Gamma_{e} \Gamma_{f} \Gamma_{g} & =4 \epsilon_{a b c d e} \eta_{f g}+\text { other terms } .
\end{aligned}
$$

Indeed, as explained in [30], whenever a $5 \mathrm{~d}$ fermion $\psi$ runs in the loop, with standard propagator and coupling to vectors of the form $A^{\mu} \bar{\psi} \Gamma_{\mu} \psi$, a contribution to the effective Chern-Simons coupling is found. Second of all, we claim that massive Kaluza-Klein modes of tensors can contribute to the parity violating part of the loop amplitude. On very general grounds, an electric coupling to the graviphoton $A^{0}$ is expected for all excited Kaluza-Klein modes. Moreover, the epsilon tensor can enter the diagram by means of a term of the form $B \wedge d B$ in the 5 d effective action.

We are now in a position to state our claim about the quantum origin of terms (5.14): they are generated by 5d one-loop diagrams with three external vectors and massive chiral modes running in the loop. In order for this mechanism to work, we have to show that the fields in the three massive families listed above interact with $5 \mathrm{~d}$ vectors in the correct way such that the result of [30] can be applied. A thorough derivation of (5.14) from one-loop calculation in $5 \mathrm{~d}$ dimensions is beyond the scope of this paper, and is left 
for further investigation in future work. Nonetheless, we can give a schematic illustration of the source of the relevant couplings and mass terms for the massive fermions in the two families listed above. Massive modes of tensors would deserve further discussion, and the authors hope to come back soon to this subject.

\subsection{Origin of the one-loop Chern-Simons couplings}

We start discussing fermionic Kaluza-Klein modes. Let $\hat{\psi}_{( \pm)}$denote a general 6 d spinor of given chirality. It is an 8-component spinor with complex entries, but the number of degrees of freedom is halved by restriction to definite chirality. This counting agrees with the number of degrees of freedom of the (off-shell) $5 \mathrm{~d}$ reduced spinor $\psi$, which can be represented as a 4-component vector with complex entries.

We can be more explicit. A representation of $6 \mathrm{~d}$ gamma matrices $\hat{\Gamma}_{\hat{a}},\left\{\hat{\Gamma}_{\hat{a}}, \hat{\Gamma}_{\hat{b}}\right\}=2 \hat{\eta}_{\hat{a} \hat{b}}$, $\hat{a}, \hat{b}=0,1, \ldots, 5$ can be found, such that

$$
\hat{\Gamma}_{a}=\sigma_{1} \otimes \Gamma_{a}, \quad \hat{\Gamma}_{5}=\sigma_{2} \otimes \mathbb{I}_{4} .
$$

In these equations, $\sigma_{i}$ are the usual Pauli matrices, while $\Gamma_{a},\left\{\Gamma_{a}, \Gamma_{b}\right\}=2 \eta_{a b}, a, b=$ $0,1, \ldots, 4$ are $5 \mathrm{~d}$ gamma matrices, satisfying

$$
i \Gamma_{0} \Gamma_{1} \Gamma_{2} \Gamma_{3} \Gamma_{4}=\mathbb{I}_{4}
$$

As a result, the $6 \mathrm{~d}$ chirality matrix is simply given by

$$
\hat{\Gamma}=\hat{\Gamma}_{0} \hat{\Gamma}_{1} \hat{\Gamma}_{2} \hat{\Gamma}_{3} \hat{\Gamma}_{4} \hat{\Gamma}_{5}=\sigma_{3} \otimes \mathbb{I}_{4}
$$

We can thus write $\hat{\psi}_{( \pm)}$in the factorized form

$$
\hat{\psi}_{( \pm)}=\iota_{( \pm)} \otimes \psi
$$

where $\iota_{( \pm)}$is a unit vector in $\mathbb{C}^{2}$, such that $\sigma_{3} \iota_{( \pm)}= \pm \iota_{( \pm)}$, and $\psi$ is a $5 \mathrm{~d}$ spinor.

Using these conventions, dimensional reduction of the $6 \mathrm{~d}$ standard kinetic term for $\hat{\psi}_{( \pm)}$yields 17

$$
\int d^{6} \hat{x} \hat{\bar{\psi}}_{( \pm)} \hat{\Gamma}^{\hat{\mu}} \hat{\partial}_{\hat{\mu}} \hat{\psi}_{( \pm)}=2 \pi \sum_{n \in \mathbb{Z}} \int d^{5} x r\left\{\bar{\psi}^{(n)} \Gamma^{\mu} \partial_{\mu} \psi^{(n)} \mp \frac{n}{r} \bar{\psi}^{(n)} \psi^{(n)}+i n A_{\mu}^{0} \bar{\psi}^{(n)} \Gamma^{\mu} \psi^{(n)}\right\}
$$

On the left hand side, a hat denotes $6 \mathrm{~d}$ gamma matrices, indices, and coordinates. The modes $\psi^{(n)}$ of the fermion $\psi$ are defined as in (5.15). On the right hand side, we find a result consistent with the general features of Kaluza-Klein models on a circle. In fact, the $n$-th excited Kaluza-Klein mode has a mass proportional to $n$ and is electrically charged with respect to the vector $A^{0}$. The charge is proportional to $n$ as well.

\footnotetext{
${ }^{17}$ In order to keep the argument simple, we work in a flat background and we do not Weyl rescale the 5 d metric.
} 
We can now turn to fermions in the vector multiplets. Let $\hat{\lambda}$ be a $6 \mathrm{~d}$ spinor in the adjoint representation of the simple gauge group $G$. Its gauge-covariant derivative is given by

$$
\hat{D} \hat{\lambda}=d \hat{\lambda}+[\hat{A}, \hat{\lambda}]
$$

where $\hat{A}$ are the non-Abelian $6 \mathrm{~d}$ vectors introduced in section 3.1. In order to keep the discussion as simple as possible, we restrict our attention to Kaluza-Klein zero-modes only in this paragraph. As a consequence, dimensional reduction of the $6 \mathrm{~d}$ kinetic term for $\hat{\lambda}$ is of the form

$$
\int d^{6} \hat{x} \operatorname{tr}\left(\hat{\bar{\lambda}} \hat{\Gamma}^{\hat{\mu}} \hat{D}_{\hat{\mu}} \hat{\lambda}\right)=2 \pi \int d^{5} x r\left\{\operatorname{tr}\left(\bar{\lambda} \Gamma^{\mu} D_{\mu} \lambda\right)+\frac{i}{r} \operatorname{tr}(\bar{\lambda}[\zeta, \lambda])\right\} .
$$

On the right hand side, $D \lambda=d \lambda+[A, \lambda]$ is the $5 \mathrm{~d}$ gauge-covariant derivative, while $\zeta$ is the adjoint scalar introduced in the Ansatz (3.28). Note that the sign of the last term is determined by the requirement of left-handedness for the gaugini, and that no $A_{0}$-coupling emerges for the Kaluza-Klein zero-modes precisely thanks to the shift of $5 \mathrm{~d}$ vectors described by (3.28). When the gauge symmetry is spontaneously broken to the Coulomb branch, the scalars $\zeta$ acquire a non-vanishing VEV orthogonal to the Cartan subalgebra. Furthermore, commutators $[A, \lambda],[\zeta, \lambda]$ vanish for the components of $\lambda$ lying in this subalgebra. However, they are non-trivial for the components orthogonal to it. These components receive a mass from the second term in (5.25), while the first term in the same equation provides electric coupling to the Abelian vectors $A^{i}$ associated to the generators of the Cartan subalgebra. We can thus see that Higgsed gaugini have the correct coupling to generate the effective Chern-Simons interaction under examination.

A similar argument can be used to conclude that charged hyperini can run in the loop and furnish a non-vanishing contribution. More precisely, dimensional reduction of their kinetic term gives

$$
\int d^{6} \hat{x} \operatorname{tr}\left[h_{U V} \hat{\bar{\psi}}^{U} \hat{\Gamma}^{\hat{\mu}}\left(\hat{\mathcal{D}}_{\hat{\mu}} \hat{\psi}\right)^{V}\right]=2 \pi \int d^{5} x r\left\{h_{U V} \bar{\psi}^{U} \Gamma^{\mu}\left(\mathcal{D}_{\hat{\mu}} \psi\right)^{V}-\frac{i}{r} h_{U V} \bar{\psi}^{U} \zeta^{I}\left(T_{I}^{\mathbf{R}} \psi\right)^{V}\right\} .
$$

In this expression, the $6 \mathrm{~d}$ covariant derivative of the hyperino is defined as

$$
\left(\hat{\mathcal{D}}_{\hat{\mu}} \hat{\psi}\right)^{U}=\hat{\nabla}_{\hat{\mu}} \hat{\psi}^{U}+\hat{A}_{\hat{\mu}}^{I}\left(T_{I}^{\mathbf{R}} \hat{\psi}\right)^{U}
$$

and an analogous expression is understood for the $5 \mathrm{~d}$ covariant derivative on the right hand side. Note that the sign of the last term has changed with respect to the gaugino reduction, because hyperini are right-handed. Upon spontaneous gauge symmetry breaking to the Coulomb branch, this term provides a mass for charged hyperini, while neutral hyperini are unaffected and remain in the massless $5 \mathrm{~d}$ spectrum.

The reader might wonder whether there are massive fermions which are electrically coupled to vectors $A^{\alpha}$. Our analysis suggests that this is not the case. A thorough explanation would require dimensional reduction of the full $6 \mathrm{~d}$ pseudo-action, including fermionic terms. Such a pseudo-action can be found e.g. in [6]. However, it is crucial to recall that $5 \mathrm{~d}$ vectors $A^{\alpha}$ are obtained by dimensional reduction of $6 \mathrm{~d}$ two-forms $\hat{B}^{\alpha}$. 
Such two-forms enter the $6 \mathrm{~d}$ action in a qualitatively different way as $6 \mathrm{~d}$ vectors. Geometrically, they are not connection forms, and cannot be used to build $6 \mathrm{~d}$ covariant derivatives. Therefore, the reduced $5 \mathrm{~d}$ action lacks electric couplings of vectors $A^{\alpha}$ to fermions. Nonetheless, different couplings are possible, which can be referred to as magnetic. They read schematically $m_{\alpha} \bar{\psi} \Gamma^{\mu \nu} F_{\mu \nu}^{\alpha} \psi$ where $\psi$ stands for a $5 \mathrm{~d}$ fermion. Even though these interactions may play a role in the full one-loop $5 \mathrm{~d}$ effective action, in the absence of electric vertices they are not able to generate contributions to the ChernSimons couplings.

It is interesting to point out the connection between this argument and the shift of vectors performed in (4.18). As explained in section 4.1, this shift is crucial to identify properly $5 \mathrm{~d}$ vectors coming from $6 \mathrm{~d}$ two-forms. As we can see by comparing (4.17) and (4.20), the field redefinition (4.18) is such that in the cubic potential $\mathcal{N}^{\mathrm{M}}$ the term $R^{2} L^{\alpha}$ gets replaced by the term $R \xi^{i} \xi^{j}$. As argued in the previous paragraph, it would be impossible to generate the former term using $5 \mathrm{~d}$ fermion loops, while in the following we will show how the latter term can emerge from such Feynman diagrams.

After these general remarks about massive fermions in the 5 d theory, let us discuss in more detail each term in (5.14). The first term corresponds to a Chern-Simons coupling of the form $A^{0} \wedge F^{0} \wedge F^{0}$. As we argued above, Kaluza-Klein modes are the fields which are electrically charged under $A^{0}$. We therefore claim that this 5 d interaction is generated by diagrams in which Kaluza-Klein excited modes coming from reduction of all chiral 6d fields can run in the loops. In order to get a finite result, the sum over modes has to be suitably regularized, e.g. by means of the Riemann zeta function. We expect the outcome of the computation to be independent of the specific regularization scheme chosen, since it describes a physical observable. It is intriguing to recall at this point the interplay between the $6 \mathrm{~d}$ anomaly coefficients and the numbers of multiplets in $6 \mathrm{~d}$ the theory. In particular, we can consider equations (B.4) and (B.6), which we record here again,

$$
n_{H}-n_{V}=273-29 n_{T}, \quad \Omega_{\alpha \beta} a^{\alpha} a^{\beta}=9-n_{T} .
$$

These relations can be combined with the identification $K^{\alpha}=a^{\alpha}$ found in the previous subsection and strongly suggest that the prefactor of the first term in (5.14) can be extracted from $5 \mathrm{~d}$ loop computations involving all species of chiral fields of the theory. Each species gives a contribution proportional to number of the corresponding $5 \mathrm{~d}$ multiplets. Note that the relation between $5 \mathrm{~d}$ and $6 \mathrm{~d}$ multiplets has been worked out in section 3.4 , see (3.51), (3.52).

The next term in (5.14) corresponds to a Chern-Simons vertex of the form $A^{0} \wedge F^{i} \wedge F^{j}$. In order to reproduce this effective coupling using $5 \mathrm{~d}$ one-loop diagrams, we need fermions which are electrically coupled both to the Kaluza-Klein vector $A^{0}$ and to the Abelian vectors $A^{i}$ in the Coulomb branch. Our discussion above singles out Kaluza-Klein modes of Higgsed gaugini and charged hyperini as natural candidates to run in the loop.

Finally, we focus our attention on the last term in (5.14), which gives rise to a ChernSimons term $A^{i} \wedge F^{j} \wedge F^{k}$. We identify the source of this coupling in the Higgsed gaugini and the massive charged hyperini. The one-loop effect due to these fermions has been 
computed 31] for a $5 \mathrm{~d} \mathcal{N}=2$ supersymmetric gauge theory decoupled from gravity. The full result for the purely gauge part of the $5 \mathrm{~d}$ cubic potential $\mathcal{N}$, including quantum corrections, reads

$$
\mathcal{N}^{\text {gauge }}=\frac{1}{2} m_{0} C_{i j} \xi^{i} \xi^{j}+\frac{1}{6} c_{\text {class }} d_{i j k} \xi^{i} \xi^{j} \xi^{k}+\frac{1}{12}\left(\sum_{\mathbf{R}}|\mathbf{R} \cdot \xi|^{3}-\sum_{f} \sum_{\mathbf{w} \in \mathbf{W}_{f}}\left|\mathbf{w} \cdot \xi+m_{f}\right|^{3}\right)
$$

In this equation $\xi$ is a vector whose component are the scalar fields $\xi^{i}$ associated to vectors $A^{i}$. In $\xi \cdot \mathbf{R}$ it is contracted with a root of the simple gauge group $G$, while in $\xi \cdot \mathbf{w}$ it contracts with a weight of a the representation in which the charged fermions transform. The first sum in (5.28) runs over all the roots of $G$, and arises from integrating out the Higgsed gaugini, i.e. the fermionic partners of massive W-bosons. The second sum in (5.28) runs over all massive charged fermions $f$ and all weights in $\mathbf{W}_{f}$, i.e. all elements of the set of weights of the representation in which the fermion $f$ transforms. $m_{f}$ is the classical mass of the fermion $f$. Finally, the group theoretical invariants $C_{i j}$ and $d_{i j k}$ are given by

$$
C_{i j}=\operatorname{tr} T_{i} T_{j}, \quad d_{i j k}=\frac{1}{2} \operatorname{tr} T_{i}\left(T_{j} T_{k}+T_{k} T_{j}\right)
$$

To apply the formula (5.28) to our $6 \mathrm{~d} / 5 \mathrm{~d}$ compactification, we recall the classical expression (5.5) for $\mathcal{N}^{\mathrm{F}}$. This leads to the identification

$$
m_{0}=-8 M^{\alpha} b^{\beta} \Omega_{\alpha \beta}, \quad c_{\text {class }}=0
$$

where we have used the fact that upon decoupling gravity the $M^{\alpha}$ are simply parameters. Following the discussion of section 5.1 this matches the classical M-theory result. A careful comparison of the loop terms in (5.28) and the intersection numbers $\mathcal{V}_{i j k}$ of the resolved Calabi-Yau threefold $\tilde{Y}_{3}$ would require the introduction of new technical tools and lies out of the main line of development of this section. However, let us stress that the reader can find a detailed discussion of this point in [26], appendix A: as explained there, the match can be performed successfully in many examples of Calabi-Yau threefolds with $S U(N)$ singularities. The classical mass $m_{f}$ is zero in this case.

In summary, we are confident that all terms in the M-theory expression (5.14) arise from one-loop quantum corrections in the $6 \mathrm{~d} / 5 \mathrm{~d}$ dual picture. Moreover, it is tempting to extend this analysis to some higher-derivative couplings which appear naturally in the M-theory reduction on a Calabi-Yau threefold, but seem to be absent in the reduction of $6 \mathrm{~d}$ supergravity on a circle. Since we have not addressed the problem of the full reduction of higher-derivative actions, we limit ourselves to an example. In section 4.2 we have seen that M-theory higher-curvature correction induce a term (4.27) which has a non-vanishing contribution involving the Kaluza-Klein vector $A^{0}$. It is proportional to the shifted component $\check{c}_{0}$ of the second Chern class of the Calabi-Yau threefold $c_{2}\left(Y_{3}\right)$ and reads schematically

$$
A^{0} \wedge \operatorname{tr} \mathcal{R} \wedge \mathcal{R}
$$


and corresponds to an amplitude with one Kaluza-Klein vector $A^{0}$ and two $5 \mathrm{~d}$ gravitons. It is impossible to extract such a coupling from the higher-curvature Green-Schwarz term (3.16) in the $6 \mathrm{~d}$ pseudo-action. Hence, we are led to claim that on the $6 \mathrm{~d} / 5 \mathrm{~d}$ side this interaction emerges as quantum effect, in a similar fashion as the $A^{0} \wedge F^{0} \wedge F^{0}$ coupling analysed above. In particular, since the tensorial structure of this vertex involves the totally antisymmetric symbol $\epsilon^{\mu \nu \rho \sigma \lambda}$, we can apply the same argument used above and infer that the only non-vanishing contributions to this coupling are due to massive modes from the reduction of $6 \mathrm{~d}$ chiral fields. Given the universality of gravitational interactions and Kaluza-Klein couplings involving $A^{0}$, it is natural to expect that all species contribute to this amplitude. A more systematic treatment of this issue is not possible in the context of the present paper, and the authors regard it as possible subject for further research.

\section{Conclusions}

In this paper we derived the $6 \mathrm{~d}(1,0)$ effective action of F-theory compactified on a singular elliptically fibred Calabi-Yau manifold $Y_{3}$. Our strategy was to use an M-theory compactification on the resolved space $\tilde{Y}_{3}$, and compare the effective 5 d action with a general $6 \mathrm{~d}$ action reduced on a circle. We included an extensive discussion of $5 \mathrm{~d}$ one-loop corrections to the Chern-Simons term and their interplay with the $6 \mathrm{~d}$ anomaly conditions.

In the first part of this work we performed the circle reduction of a general $6 \mathrm{~d}(1,0)$ supergravity theory with a non-Abelian gauge group $G$. We performed the Kaluza-Klein reduction in the non-Abelian phase and later discussed the modifications when the effective $5 \mathrm{~d}$ theory is considered on the Coulomb branch. We argued that the charged hypermultiplets and the vector multiplets containing the $\mathrm{W}$-bosons are massive in this phase and need to be integrated out when comparing with an M-theory reduction on $\tilde{Y}_{3}$. Moreover, we presented a careful treatment of the self-dual and anti-self-dual tensors present in a general $6 \mathrm{~d}$ theory. While we used a $6 \mathrm{~d}$ pseudo-action, which has to be accompanied by the self-duality conditions on the level of the equations of motion, we showed in detail that in the Kaluza-Klein reduced theory the self-duality can be imposed on the level of the action now relating $5 \mathrm{~d}$ vectors and tensors. However, due to the fact that the $6 \mathrm{~d}$ theory requires an anomaly cancelling Green-Schwarz term, the resulting 5d theory is also classically non-gauge invariant. We showed that its vector sector can nevertheless be encoded by a single real function $\mathcal{N}^{\mathrm{F}}$ which is homogeneous of degree three. However, $\mathcal{N}^{\mathrm{F}}$ contains a non-polynomial term which is required to encode the complete $5 \mathrm{~d}$ metric for the vectors and enforce $\mathcal{N}^{\mathrm{F}}=1$ consistent with the $6 \mathrm{~d}$ supergravity constraint $\Omega_{\alpha \beta} j^{\alpha} j^{\beta}=1$ imposing a condition on the real scalars $j^{\alpha}$ in the tensor multiplets. The non-polynomial term is not present in a standard $5 \mathrm{~d} \mathcal{N}=2$ supergravity theory and induces a non-gauge invariant term. We identify this term as a one-loop counterterm. The 6d Green-Schwarz term also contains a higher curvature coupling and we presented a partial dimensional $5 \mathrm{~d}$ reduction of this term.

In the second part of this paper we compared the circle reduced action with the $5 \mathrm{~d}$ 
effective action of M-theory on a Calabi-Yau threefold $\tilde{Y}_{3}$. To extract the $5 \mathrm{~d} \mathcal{N}=2$ characteristic data in a geometric regime one has to work with the resolved threefold $\tilde{Y}_{3}$, where both the gauge group singularities at co-dimension one in $B_{2}$, as well as the matter singularities at co-dimension two in $B_{2}$ are resolved. Accordingly all M2-brane states wrapped on cycles in $\tilde{Y}_{3}$ are massive and do not arise as dynamical degrees of freedom in the $5 \mathrm{~d}$ effective theory. However, the $5 \mathrm{~d}$ effective action of M-theory on $\tilde{Y}_{3}$ contains terms which arise by consistently integrating out these massive states. To disentangle these from the terms present in the classical $6 \mathrm{~d} / 5 \mathrm{~d}$ reduction we introduced a scaling limit corresponding to the F-theory limit. The finite terms in the M-theory reduction are readily matched with the general $6 \mathrm{~d} / 5 \mathrm{~d}$ result. This enabled us to determine the core characteristic data required to evaluate the $6 \mathrm{~d}(1,0)$ F-theory effective action in terms of the geometric data of $\tilde{Y}_{3}$. Also dimensionally reducing the known M-theory higher curvature terms we were able to extract from a $5 \mathrm{~d}$ comparison the integral vectors $\left(a^{\alpha}, b^{\alpha}\right)$ encoding all $6 \mathrm{~d}$ anomalies.

In the treatment of the massive states we have discovered an intriguing interplay of $5 \mathrm{~d}$ one-loop corrections and $6 \mathrm{~d}$ anomalies. In fact, since the M-theory reduction is on the resolved $\tilde{Y}_{3}$, all M2-brane states wrapped on the resolving $\mathbb{P}^{1}$-fibres are massive. These M2-brane states are dual in the F-theory limit of M-theory to the $6 \mathrm{~d}$ charged hypermultiplets, and $6 \mathrm{~d}$ vector multiplets containing the $\mathrm{W}$-bosons. Accordingly, one can only compare the $5 \mathrm{~d}$ theories if these massive states are consistently integrated out also in the circle reduced theory. This is equally true for the M2-brane states on the elliptic fibre itself which are massive for a finite fibre volume. Using the M-theory to F-theory lift we identify these modes as certain Kaluza-Klein modes. More generally, this implies that also massive Kaluza-Klein modes have to be integrated out consistently in the circle reduction to compare the $5 \mathrm{~d}$ result with the M-theory reduction. We have focused in this work on the investigation of the $5 \mathrm{~d}$ Chern-Simons couplings which only receive corrections due to massive $5 \mathrm{~d}$ modes of $6 \mathrm{~d}$ chiral fields in one-loop diagrams. The investigation of the various couplings allowed us to identify the one-loop diagrams generating the classically absent couplings. In particular, we argued that the couplings $A^{0} \wedge F^{0} \wedge F^{0}$ and $A^{0} \wedge \operatorname{tr} \mathcal{R} \wedge \operatorname{tr} \mathcal{R}$ are generated by integrating out massive Kaluza-Klein modes. We expect that both fermions and tensors can run in this loop diagram. A detailed account of possible fermionic coupling has been given, while we leave a proper discussion of massive tensors for future investigation. More familiar, are the couplings $A^{i} \wedge F^{i} \wedge F^{j}$, which are generated by integrating out massive hyperini and gaugini. The mixed terms, such as $A^{0} \wedge F^{i} \wedge F^{j}$, are induced by combining the vertices and propagators of both sets of massive fermionic modes. We believe that comparing the resulting coefficient functions to the geometric M-theory result leads to a $5 \mathrm{~d}$ derivation of the $6 \mathrm{~d}$ anomaly cancellation conditions. While we have summarized the necessary tools to perform these one-loop integrals, we leave the explicit evaluation of all Chern-Simons coefficients to future work.

There are various interesting directions for further research. Firstly, one can extend the classical reduction on both the $6 \mathrm{~d} / 5 \mathrm{~d}$ action and the M-theory side to more then one 
non-Abelian gauge group. Also the extension to include Abelian $U(1)$ gauge groups is desirable. Additional $U(1)$ gauge fields will modify the $6 \mathrm{~d}$ anomaly constraints and lead to

new insights about the interplay of resolved geometries and 6d gauge theories 18 Beyond the classical analysis it would be important to extend the study of loop corrections to all terms in the $5 \mathrm{~d}$ action obtained by circle reduction. This includes a detailed study of the metric for the neutral hypermultiplets. Also a evaluation of the coefficient of the $5 \mathrm{~d}$ higher curvature corrections, generated at the quantum level, will be desirable. Comparing the results with the coefficients $\check{c}_{i}, \check{c}_{0}$ predicted by the geometry of $\tilde{Y}_{3}$ on the M-theory side, will be a non-trivial test of the F-theory limit and its consistency with $6 \mathrm{~d}$ anomaly cancellation. Reversely, one might also be able to use known $6 \mathrm{~d}$ higher curvature terms to infer additional terms in the 11d supergravity action. This is particularly interesting since the $6 \mathrm{~d} / 5 \mathrm{~d}$ Kaluza-Klein vector is part of the M-theory three-form.

Let us close by noting that in this work we have only dealt with Abelian tensor fields in the $6 \mathrm{~d}$ action. We have found that in this case the couplings of the form $A^{\alpha} \wedge F^{\beta} \wedge F^{\gamma}$ are not generated in the $5 \mathrm{~d}$ effective theory. In a future project we hope to generalize the transdimensional treatment of tensors to the non-Abelian case. It will be interesting to investigate how the various terms expected for non-Abelian tensors are generated in the M-theory picture.

Acknowledgments: We would like to thank Hirotaka Hayashi, Stefan Hohenegger, Denis Klevers, Albrecht Klemm, Noppadol Mekareeya, Daniel Park, Raffaele Savelli, Maximilian Schmidt-Sommerfeld and Wati Taylor for interesting discussions. This work was supported by a research grant of the Max Planck Society.

\section{Appendices}

\section{A Notations and conventions}

For every spacetime dimension $d$, we adopt the mostly plus convention for the metric $g_{\mu \nu}$, and the $(+++)$ conventions of [50] for the Riemann tensor: explicitly,

$$
\begin{aligned}
\Gamma_{\mu \nu}^{\rho} & =\frac{1}{2} g^{\rho \sigma}\left(\partial_{\mu} g_{\nu \sigma}+\partial_{\nu} g_{\mu \sigma}-\partial_{\sigma} g_{\mu \nu}\right) \\
R_{\tau \mu \nu}^{\lambda} & =\partial_{\mu} \Gamma_{\nu \tau}^{\lambda}-\partial_{\nu} \Gamma_{\mu \tau}^{\lambda}+\Gamma_{\mu \alpha}^{\lambda} \Gamma_{\nu \tau}^{\alpha}-\Gamma_{\nu \alpha}^{\lambda} \Gamma_{\mu \tau}^{\alpha} \\
R_{\mu \nu} & =R_{\mu \lambda \nu}^{\lambda}, \quad R=R_{\mu \nu} g^{\mu \nu} .
\end{aligned}
$$

\footnotetext{
${ }^{18}$ See ref. [20] [21] for recent progress in this direction.
} 
We use $\epsilon_{\mu_{1} \ldots \mu_{d}}$ to denote the Levi-Civita tensor, and use the metric to raise its indices. It is defined in such a way that, in any coordinate system $\left(x^{0}, x^{1}, \ldots, x^{d-1}\right)$,

$$
\epsilon_{01 \ldots(d-1)}=+\sqrt{-\operatorname{det} g_{\mu \nu}} .
$$

Note that the following identity holds for arbitrary $k=0, \ldots, d$ :

$$
\epsilon_{\mu_{1} \ldots \mu_{k} \lambda_{k+1} \ldots \lambda_{d}} \epsilon^{\nu_{1} \ldots \nu_{k} \lambda_{k+1} \ldots \lambda_{d}}=-k !(d-k !) \delta_{\left[\mu_{1}\right.}^{\nu_{1}} \ldots \delta_{\left.\mu_{k}\right]}^{\nu_{k}}
$$

Differential $p$-forms are expanded on the basis of differential of the coordinates as

$$
\lambda=\frac{1}{p !} \lambda_{\mu_{1} \ldots \mu_{p}} d x^{\mu_{1}} \wedge \cdots \wedge d x^{\mu_{p}}
$$

so that the wedge product of a $p$ - and a $q$-form satisfies

$$
(\alpha \wedge \beta)_{\mu_{1} \ldots \mu_{p+q}}=\frac{(p+q) !}{p ! q !} \alpha_{\left[\mu_{1} \ldots \mu_{p}\right.} \beta_{\left.\mu_{p+1} \ldots \mu_{p+q}\right]}
$$

Exterior differentiation of a $p$-form is given by

$$
(d \alpha)_{\mu_{0} \ldots \mu_{p}}=(p+1) \partial_{\left[\mu_{0}\right.} \alpha_{\left.\mu_{1} \ldots \mu_{p}\right]} .
$$

The Hodge dual of $p$-form in real coordinates and arbitrary spacetime dimension $d$ is defined by expression

$$
(* \alpha)_{\mu_{1} \ldots \mu_{d-p}}=\frac{1}{p !} \alpha^{\nu_{1} \ldots \nu_{p}} \epsilon_{\nu_{1} \ldots \nu_{p} \mu_{1} \ldots \mu_{d-p}}
$$

As a consequence,

$$
\alpha \wedge * \beta=\frac{1}{p !} \alpha_{\mu_{1} \ldots \mu_{p}} \beta^{\mu_{1} \ldots \mu_{p}} * 1
$$

holds identically for arbitrary $p$-forms $\alpha, \beta$.

\section{B Anomalies in 6d supergravity}

In subsection 3.1 we mentioned generalized Green-Schwarz mechanism [12, 13, 18] for anomaly cancellation in a $6 \mathrm{~d}$ supergravity model with simple gauge group $G$. In this appendix we review this mechanism in the more general case in which the gauge group is the direct product of several simple groups $G_{i}$. Possible Abelian factors are not take into account.

In $6 \mathrm{~d}$ models, tree-level exchange of $\hat{B}^{\alpha}$ quanta can counterbalance one-loop anomalous diagrams. For this to be possible, the total anomaly polynomial must be of the form

$$
\hat{I}_{8}=\frac{1}{2} \Omega_{\alpha \beta} \hat{X}_{4}^{\alpha} \wedge \hat{X}_{4}^{\beta},
$$

where

$$
\hat{X}_{4}^{\alpha}=\frac{1}{2} a^{\alpha} \operatorname{tr} \hat{\mathcal{R}} \wedge \hat{\mathcal{R}}+\sum_{i} 2 b_{i}^{\alpha} \lambda_{i}^{-1} \operatorname{tr}_{f} \hat{F}_{i} \wedge \hat{F}_{i}
$$


In these expressions $a^{\alpha}, b_{i}^{\alpha}$ transform as vectors in the space $\mathbb{R}^{1, T}$ with symmetric inner product $\Omega_{\alpha \beta}$. Furthermore, $\operatorname{tr}_{f}$ of $\hat{F}_{i}^{2}$ denotes the trace in the fundamental representation, and $\lambda_{i}$ are normalization constants depending on the type of each simple group factor. In the main text, this constant is always reabsorbed in the normalization of the trace of field strengths, $\operatorname{tr}=\lambda^{-1} \operatorname{tr}_{f}$. We refer the reader to [19] for the value of $\lambda$ for various simple gauge groups.

If condition (B.1) is met, the theory can be made anomaly-free by introduction of the generalized Green-Schwarz term

$$
\hat{S}^{\mathrm{GS}}=-\int_{\mathcal{M}_{5}} \frac{1}{2} \Omega_{\alpha \beta} \hat{B}^{\alpha} \wedge \hat{X}_{4}^{\beta} .
$$

By computation of the anomaly polynomial $\hat{I}_{8}$ in terms of the chiral matter content and comparison with the factorized form (B.1), the following necessary conditions for anomaly cancellation are found:

$$
\begin{aligned}
& n_{H}-n_{V}=273-29 n_{T} \\
& 0=B_{\mathrm{adj}}^{i}-\sum_{\mathbf{R}} x_{\mathbf{R}}^{i} B_{\mathbf{R}}^{i} \\
& \Omega_{\alpha \beta} a^{\alpha} a^{\beta}=9-n_{T} \\
& -\Omega_{\alpha \beta} a^{\alpha} b_{i}^{\beta}=\frac{1}{6} \lambda_{i}\left(\sum_{\mathbf{R}} x_{\mathbf{R}}^{i} A_{\mathbf{R}}^{i}-A_{\text {adj }}^{i}\right) \\
& \Omega_{\alpha \beta} b_{i}^{\alpha} b_{i}^{\beta}=\frac{1}{3} \lambda_{i}^{2}\left(\sum_{\mathbf{R}} x_{\mathbf{R}}^{i} C_{\mathbf{R}}^{i}-C_{\text {adj }}^{i}\right) \quad \text { (no sum over } i \text { ) } \\
& \Omega_{\alpha \beta} b_{i}^{\alpha} b_{j}^{\beta}=\lambda_{i} \lambda_{j} \sum_{\mathbf{R S}} x_{\mathbf{R S}}^{i j} A_{\mathbf{R}}^{i} A_{\mathbf{S}}^{j} \quad(i \neq j) .
\end{aligned}
$$

In these equations, $n_{H}, n_{V}, n_{T}$ are the numbers of hyper-, vector and tensor multiplets in the model, $A_{\mathbf{R}}, B_{\mathbf{R}}, C_{\mathbf{R}}$ are group theory coefficients defined through

$$
\begin{aligned}
& \operatorname{tr}_{\mathbf{R}} \hat{F}^{2}=A_{\mathbf{R}} \operatorname{tr}_{f} \hat{F}^{2} \\
& \operatorname{tr}_{\mathbf{R}} \hat{F}^{4}=B_{\mathbf{R}} \operatorname{tr}_{f} \hat{F}^{4}+C_{\mathbf{R}}\left(\operatorname{tr}_{f} \hat{F}^{2}\right)^{2},
\end{aligned}
$$

and $x_{\mathbf{R}}^{i}, x_{\mathbf{R S}}^{i j}$ denote the number of matter fields that transform in the irreducible representation $\mathbf{R}$ of gauge group factor $G_{i}$, and $(\mathbf{R}, \mathbf{S})$ of $G_{i} \times G_{j}$, respectively. Note that for groups such as $S U(2)$ and $S U(3)$, which lack a fourth order invariant, $B_{\mathbf{R}}=0$ and there is no condition B.5.

\section{Two-derivative $6 \mathrm{~d}(1,0)$ supergravity on a circle}

In this appendix we discuss the dimensional reduction of $6 \mathrm{~d}(1,0)$ supergravity at twoderivative level. Our starting point is therefore (3.22), which we write down again for 
convenience,

$$
\begin{gathered}
\hat{S}^{(6)}=\int_{\mathcal{M}_{6}}+\frac{1}{2} \hat{R} \hat{*} 1-\frac{1}{4} g_{\alpha \beta} \hat{G}^{\alpha} \wedge \hat{*} \hat{G}^{\beta}-\frac{1}{2} g_{\alpha \beta} d j^{\alpha} \wedge \hat{*} d j^{\beta}-h_{U V} \hat{\mathcal{D}} q^{U} \wedge \hat{*} \hat{\mathcal{D}} q^{V} \\
-2 \Omega_{\alpha \beta} j^{\alpha} b^{\beta} \operatorname{tr} \hat{F} \wedge \hat{*} \hat{F}-\Omega_{\alpha \beta} b^{\alpha} \hat{B}^{\beta} \wedge \operatorname{tr} \hat{F} \wedge \hat{F}-\hat{V} \hat{*} 1
\end{gathered}
$$

The Kaluza-Klein Ansatz for the metric was given in (3.25), while vectors and two-forms are expanded in 5d fields according to (3.28), (3.31). Consistently with our two-derivative approximation, we omit the gravitational contribution proportional to $a^{\alpha}$ in eq. (3.31). This implies that the gravitational part is dropped in $G^{\alpha}$, too.

Standard dimensional reduction techniques can be applied to this pseudo-action, considered as a functional of both $A^{\alpha}$ and $B^{\alpha}$ independently. One computes

$$
\begin{aligned}
S_{\text {pseudo }}^{(5) \mathrm{F}}=\int_{\mathcal{M}_{5}} & +\frac{1}{2} r \tilde{R} \tilde{*} 1-\frac{1}{4} r^{3} F^{0} \wedge \tilde{*} F^{0}-\frac{1}{2} r g_{\alpha \beta} d j^{\alpha} \wedge \tilde{*} d j^{\beta}-r h_{U V} \mathcal{D} q^{U} \wedge \tilde{*} \mathcal{D} q^{V} \\
& -2 r \Omega_{\alpha \beta} j^{\alpha} b^{\beta} \operatorname{tr}\left(F-\zeta F^{0}\right) \wedge \tilde{*}\left(F-\zeta F^{0}\right)-2 r^{-1} \Omega_{\alpha \beta} j^{\alpha} b^{\beta} \operatorname{tr} D \zeta \wedge \tilde{*} D \zeta \\
& -\frac{1}{4} r g_{\alpha \beta} G^{\alpha} \wedge \tilde{*} G^{\beta}-\frac{1}{4} r^{-1} g_{\alpha \beta} \mathcal{F}^{\alpha} \wedge \tilde{*} \mathcal{F}^{\beta} \\
& -\frac{1}{2} \Omega_{\alpha \beta} G^{\alpha} \wedge\left(\mathcal{F}^{\beta}-F^{\beta}\right)+\Omega_{\alpha \beta} b^{\alpha} A^{\beta} \wedge \operatorname{tr} F \wedge F \\
& -2 \Omega_{\alpha \beta} b^{\alpha} b^{\beta} \omega^{\mathrm{CS}} \wedge\left(2 \operatorname{tr} \zeta F-\operatorname{tr} \zeta \zeta F^{0}\right) \\
& -2 \Omega_{\alpha \beta} b^{\alpha} b^{\beta} \operatorname{tr} \zeta A \wedge\left(\operatorname{tr} F \wedge F-2 \operatorname{tr} \zeta F \wedge F^{0}+\operatorname{tr} \zeta \zeta F^{0} \wedge F^{0}\right) \\
& -\left[r \hat{V}+r^{-1} h_{U V} \zeta^{I} \zeta^{J}\left(T_{I}^{\mathbf{R}} q\right)^{U}\left(T_{J}^{\mathbf{R}} q\right)^{V}\right] \tilde{*} 1 .
\end{aligned}
$$

In this expression, $D \zeta=d \zeta+[A, \zeta]$ is the gauge covariant derivative for the adjoint scalars $\zeta$, while $\mathcal{D} q^{U}=d q^{U}+A^{I}\left(T_{I}^{\mathbf{R}} q\right)^{U}$ are the 5 d gauge covariant derivatives for the scalars $q^{U}$ in the hypermultiplets. Furthermore, we have introduced the shorthand notation

$$
\mathcal{F}^{\alpha}=F^{\alpha}-4 b^{\alpha} \operatorname{tr} \zeta F+2 b^{\alpha} \operatorname{tr} \zeta \zeta F^{0} .
$$

Dimensional reduction of the the self-duality constraint (3.21) gives

$$
r g_{\alpha \beta} \tilde{*} G^{\beta}=-\Omega_{\alpha \beta} \mathcal{F}^{\beta}
$$

where the minus sign comes from our Ansatz (3.31). This relation means that $A^{\alpha}$ and $B^{\alpha}$ encode the same physical degrees of freedom. Let us now discuss in detail how we can obtain a proper $5 \mathrm{~d}$ action written in terms of vectors $A^{\alpha}$ only. The first step amounts to adding a total derivative to the action above: $S^{(5) \mathrm{F}}=S_{\text {pseudo }}^{(5) \mathrm{F}}+\Delta S^{(5) \mathrm{F}}$, where

$$
\begin{aligned}
\Delta S^{(5) \mathrm{F}} & =\int_{\mathcal{M}_{5}}-\frac{1}{2} \Omega_{\alpha \beta} d B^{\alpha} \wedge F^{\beta} \\
& =\int_{\mathcal{M}_{5}}-\frac{1}{2} \Omega_{\alpha \beta} G^{\alpha} \wedge F^{\beta}+\frac{1}{2} \Omega_{\alpha \beta}\left(-A^{\alpha} F^{0}+2 b^{\alpha} \omega^{\mathrm{CS}}\right) \wedge F^{\beta}
\end{aligned}
$$


If we now consider $S^{(5) \text { F }}$ as a functional of $G^{\alpha}, A^{\alpha}$, the equations of motion ensure both the self-duality condition (C.4) and the non-standard Bianchi identity (3.38). Moreover, $G^{\alpha}$ enters $S^{(5) \mathrm{F}}$ only quadratically, and is therefore readily integrated out:

$$
\begin{aligned}
S^{(5) \mathrm{F}}=\int_{\mathcal{M}_{5}} & +\frac{1}{2} r \tilde{R} \tilde{*} 1-\frac{1}{4} r^{3} F^{0} \wedge \tilde{*} F^{0}-\frac{1}{2} r g_{\alpha \beta} d j^{\alpha} \wedge \tilde{*} d j^{\beta}-r h_{U V} \mathcal{D} q^{U} \wedge \tilde{*} \mathcal{D} q^{V} \\
& -2 r \Omega_{\alpha \beta} j^{\alpha} b^{\beta} \operatorname{tr}\left(F-\zeta F^{0}\right) \wedge \tilde{*}\left(F-\zeta F^{0}\right)-2 r^{-1} \Omega_{\alpha \beta} j^{\alpha} b^{\beta} \operatorname{tr} D \zeta \wedge \tilde{*} D \zeta \\
& -\frac{1}{2} r^{-1} g_{\alpha \beta} \mathcal{F}^{\alpha} \wedge \tilde{*} \mathcal{F}^{\beta}-\frac{1}{2} \Omega_{\alpha \beta} A^{0} \wedge F^{\alpha} \wedge F^{\beta}+2 \Omega_{\alpha \beta} b^{\alpha} A^{\beta} \wedge \operatorname{tr} F \wedge F \\
& -2 \Omega_{\alpha \beta} b^{\alpha} b^{\beta} \omega^{\mathrm{CS}} \wedge\left(2 \operatorname{tr} \zeta F-\operatorname{tr} \zeta \zeta F^{0}\right) \\
& -2 \Omega_{\alpha \beta} b^{\alpha} b^{\beta} \operatorname{tr} \zeta A \wedge\left(\operatorname{tr} F \wedge F-2 \operatorname{tr} \zeta F \wedge F^{0}+\operatorname{tr} \zeta \zeta F^{0} \wedge F^{0}\right) \\
& -\left[r \hat{V}+r^{-1} h_{U V} \zeta^{I} \zeta^{J}\left(T_{I}^{\mathbf{R}} q\right)^{U}\left(T_{J}^{\mathbf{R}} q\right)^{V}\right] \tilde{*} 1 .
\end{aligned}
$$

It is worth pointing out that $-\frac{1}{4} r g_{\alpha \beta} G^{\alpha} \wedge \tilde{*} G^{\beta}-\frac{1}{4} r^{-1} g_{\alpha \beta} \mathcal{F}^{\alpha} \wedge \tilde{*}^{\beta}$ vanishes identically after elimination of $G^{\alpha}$, and that the kinetic term for vectors $-\frac{1}{2} r^{-1} g_{\alpha \beta} \mathcal{F}^{\alpha} \wedge \tilde{*} \mathcal{F}^{\beta}$ comes from the Chern-Simons term $-\frac{1}{2} \Omega_{\alpha \beta} G^{\alpha} \wedge \mathcal{F}^{\beta}$. Moreover, the term $+2 \Omega_{\alpha \beta} b^{\alpha} A^{\alpha} \wedge \operatorname{tr} F \wedge F$ has a different prefactor because two different contributions must be taken into account: one was already present in ( $\underline{\text { C.2 }}$ ), the other one is found in $\Delta S^{(5) \mathrm{F}}$.

The last step consists of the Weyl rescaling $\tilde{g}_{\mu \nu}=r^{-2 / 3} g_{\mu \nu}$, which brings the EinsteinHilbert term in (C.7) into canonical form:

$$
\begin{aligned}
S^{(5) \mathrm{F}}=\int_{\mathcal{M}_{5}} & +\frac{1}{2} R * 1-\frac{2}{3} r^{-2} d r \wedge * d r-\frac{1}{2} g_{\alpha \beta} d j^{\alpha} \wedge * d j^{\beta} \\
& -2 r^{-2} \Omega_{\alpha \beta} j^{\alpha} b^{\beta} \operatorname{tr} D \zeta \wedge * D \zeta-h_{U V} \mathcal{D} q^{U} \wedge * \mathcal{D} q^{V} \\
& -\frac{1}{4} r^{8 / 3} F^{0} \wedge * F^{0}-\frac{1}{2} r^{-4 / 3} g_{\alpha \beta} \mathcal{F}^{\alpha} \wedge * \mathcal{F}^{\beta} \\
& -2 r^{2 / 3} \Omega_{\alpha \beta} j^{\alpha} b^{\beta} \operatorname{tr}\left(F-\zeta F^{0}\right) \wedge *\left(F-\zeta F^{0}\right) \\
& -\frac{1}{2} \Omega_{\alpha \beta} A^{0} \wedge F^{\alpha} \wedge F^{\beta}+2 \Omega_{\alpha \beta} b^{\alpha} A^{\beta} \wedge \operatorname{tr} F \wedge F \\
& -2 \Omega_{\alpha \beta} b^{\alpha} b^{\beta} \omega^{\mathrm{CS}} \wedge\left(2 \operatorname{tr} \zeta F-\operatorname{tr} \zeta \zeta F^{0}\right) \\
& -2 \Omega_{\alpha \beta} b^{\alpha} b^{\beta} \operatorname{tr} \zeta A \wedge\left(\operatorname{tr} F \wedge F-2 \operatorname{tr} \zeta F \wedge F^{0}+\operatorname{tr} \zeta \zeta F^{0} \wedge F^{0}\right) \\
& -\left[r^{-1} \hat{V}+r^{-8 / 3} h_{U V} \zeta^{I} \zeta^{J}\left(T_{I}^{\mathbf{R}} q\right)^{U}\left(T_{J}^{\mathbf{R}} q\right)^{V}\right] * 1 .
\end{aligned}
$$

As explained in subsection 3.3, we are interested in the broken phase of the theory corresponding to the Coulomb branch of the gauge sector. The fields which acquire a mass during the spontaneous breaking of gauge symmetry are omitted from the final $5 \mathrm{~d}$ effective action. These include $\mathrm{W}$-bosons and charged hypermultiplet scalars. As a consequence, the lower-case indices $u, v$ now only run over neutral hypermultiplets. For the same reason, the scalar potential is omitted. The final form of the effective action in 
the Coulomb branch thus reads

$$
\begin{aligned}
S^{(5) \mathrm{F}}=\int_{\mathcal{M}_{5}} & +\frac{1}{2} R * 1-\frac{2}{3} r^{-2} d r \wedge * d r-\frac{1}{2} g_{\alpha \beta} d j^{\alpha} \wedge * d j^{\beta} \\
& -2 r^{-2} \Omega_{\alpha \beta} j^{\alpha} b^{\beta} C_{i j} d \zeta^{i} \wedge * d \zeta^{j}-h_{u v} d q^{u} \wedge * d q^{v} \\
& -\frac{1}{4} r^{8 / 3} F^{0} \wedge * F^{0}-\frac{1}{2} r^{-4 / 3} g_{\alpha \beta} \mathcal{F}^{\alpha} \wedge * \mathcal{F}^{\beta} \\
& -2 r^{2 / 3} \Omega_{\alpha \beta} C_{i j} j^{\alpha} b^{\beta}\left(F^{i}-\zeta^{i} F^{0}\right) \wedge *\left(F^{j}-\zeta^{j} F^{0}\right) \\
& -\frac{1}{2} \Omega_{\alpha \beta} A^{0} \wedge F^{\alpha} \wedge F^{\beta}+2 \Omega_{\alpha \beta} C_{i j} b^{\alpha} A^{\beta} \wedge F^{i} \wedge F^{j} \\
& -2\left(\Omega_{\alpha \beta} b^{\alpha} b^{\beta}\right)\left(C_{k l} \zeta^{k} \zeta^{l}\right) C_{i j} \zeta^{i} A^{j} \wedge F^{0} \wedge F^{0} \\
& +2\left(\Omega_{\alpha \beta} b^{\alpha} b^{\beta}\right)\left(C_{i j} C_{k l} \zeta^{k} \zeta^{l}+2 C_{i k} C_{j l} \zeta^{k} \zeta^{l}\right) A^{i} \wedge F^{j} \wedge F^{0} \\
& -6\left(\Omega_{\alpha \beta} b^{\alpha} b^{\beta}\right) C_{(i j} C_{k) l} \zeta^{l} A^{i} \wedge F^{j} \wedge F^{k} .
\end{aligned}
$$

\section{Calabi-Yau reference formulae}

The main purpose of this appendix is fixing some notation about Calabi-Yau threefolds. Therefore, it is not meant to be complete nor self-contained. We refer the reader to e.g. [51, 52] for a more detailed account of the material covered hereafter.

A Calabi-Yau threefold $Y_{3}$ can be described locally either by means of six real coordinates $\left\{\xi^{\hat{\imath}}\right\}_{\hat{\imath}=\hat{1}, \ldots \hat{6}}$, or by means of three complex coordinates $\left\{y^{i}\right\}_{i=1,2,3}$, defined as

$$
y^{1}=\frac{1}{\sqrt{2}}\left(\xi^{\hat{1}}+i \xi^{\hat{2}}\right), \quad y^{2}=\frac{1}{\sqrt{2}}\left(\xi^{\hat{3}}+i \xi^{\hat{4}}\right), \quad y^{3}=\frac{1}{\sqrt{2}}\left(\xi^{\hat{5}}+i \xi^{\hat{6}}\right) .
$$

In the following, we will make use of complex coordinates, and their associated holomorphic indices $i, j, \ldots=1,2,3$ and antiholomorphic indices $\bar{\imath}, \bar{\jmath}, \ldots=\overline{1}, \overline{2}, \overline{3}$. Accordingly,

a complex differential form of degree $(r, s)$ is expanded on the basis of differentials of complex coordinates as

$$
\alpha=\frac{1}{r ! s !} \alpha_{i_{1} \ldots i_{r} \bar{\jmath}_{1} \ldots \bar{\jmath}_{s}} d y^{i_{1}} \wedge \cdots \wedge d y^{i_{r}} \wedge d \bar{y}^{\bar{\jmath}_{1}} \wedge \cdots \wedge d \bar{y}^{\bar{\jmath}_{s}}
$$

Being a Kähler threefold, $Y_{3}$ is endowed with an Hermitian metric $g_{i \bar{\jmath}}$, whose Kähler $(1,1)$-form $J=i g_{i \bar{\jmath}} d y^{i} \wedge d \bar{y}^{\bar{\jmath}}$ is closed. The Calabi-Yau condition ensures the existence of a globally defined, non-vanishing, holomorphic (3,0)-form, which we denote by $\Omega$. The volume form, the Kähler form and the holomorphic $(3,0)$-form are related by

$$
* 1=\frac{1}{3 !} J \wedge J \wedge J=\frac{i}{\|\Omega\|^{2}} \Omega \wedge \bar{\Omega}, \quad \text { where } \quad\|\Omega\|^{2}=\frac{1}{3 !} \Omega_{i j k} \bar{\Omega}^{i j k} .
$$

Since our definition of a Calabi-Yau threefold implies strict $S U(3)$ holonomy, the only independent Hodge numbers of $Y_{3}$ are $h^{1,1}\left(Y_{3}\right), h^{1,2}\left(Y_{3}\right)$. Let us fix our notations for the corresponding cohomology basis. 
First of all, we choose an integral cohomology basis $\left\{\omega_{\Lambda}\right\}_{\Lambda}$ for $H^{1,1}\left(Y_{3}\right)$, with $\Lambda=$ $1, \ldots, h^{1,1}\left(Y_{3}\right)$. The intersection numbers associated to this basis $\left\{\omega_{\Lambda}\right\}_{\Lambda}$ are

$$
\mathcal{V}_{\Lambda \Sigma \Theta}=\int_{Y_{3}} \omega_{\Lambda} \wedge \omega_{\Sigma} \wedge \omega_{\Theta}
$$

Second of all, we take $H^{2,1}\left(Y_{3}\right)$ to be generated by the complex cohomology basis $\left\{\chi_{\kappa}\right\}_{\kappa}$, where $\kappa=1, \ldots, h^{1,2}\left(Y_{3}\right)$. It is also useful to consider an integral basis $\left\{\alpha_{K}, \beta^{K}\right\}_{K}$ for the middle cohomology $H^{3}\left(Y_{3}\right)$, with $K=1, \ldots, h^{1,2}\left(Y_{3}\right)+1$. Since three-forms anticommute, it is natural to introduce a symplectic structure on $H^{3}\left(Y_{3}\right)$ choosing $\alpha_{K}, \beta^{K}$ in such a way that

$$
\int_{Y_{3}} \alpha_{K} \wedge \beta^{L}=\delta_{L}^{K}
$$

In order to discuss the metric on the moduli space of neutral hypermultiplets, we need to introduce matrices $A_{K}{ }^{L}, B_{K L}, C^{K L}$, such that

$$
* \alpha_{K}=A_{K}{ }^{L} \alpha_{L}+B_{K L} \beta^{L}, \quad * \beta^{K}=C^{K L} \alpha_{L}-A_{L}{ }^{K} \beta^{L},
$$

where $*$ represents the Hodge star in $Y_{3}$. These matrices can be conveniently expressed in terms of a symmetric, complex matrix $\mathcal{M}$ :

$$
\begin{aligned}
& A_{K}{ }^{L}=(\operatorname{Re} \mathcal{M})_{K H}(\operatorname{Im} \mathcal{M})^{-1 H L}, \\
& B_{K L}=-(\operatorname{Im} \mathcal{M})_{K L}-(\operatorname{Re} \mathcal{M})_{K H}(\operatorname{Im} \mathcal{M})^{-1 H M}(\operatorname{Re} \mathcal{M})_{M L}, \\
& C^{K L}=(\operatorname{Im} \mathcal{M})^{-1 K L} .
\end{aligned}
$$

Let us now give a brief account on the moduli space of Calabi-Yau threefold $Y_{3}$. It is known that it can be written locally in a factorized form as the product of the Kähler moduli space and the complex structure moduli space: $\mathcal{M}=\mathcal{M}_{\mathrm{K}} \times \mathcal{M}_{\mathrm{cs}}$. We discuss each factor in turn.

On the one hand, the Kähler moduli space $\mathcal{M}_{\mathrm{K}}$ is parameterized by the Kähler moduli $v^{\Lambda}$ which appear in the expansion of the Kähler form $J$ on the basis $\left\{\omega_{\Lambda}\right\}_{\Lambda}$,

$$
J=v^{\Lambda} \omega_{\Lambda} .
$$

Fluctuations of the Kähler moduli encode information about the variation of the mixed components of the Ricci-flat metric as we move around in the moduli space of $Y_{3}$, as specified by

$$
\delta g_{i \bar{\jmath}}=-i\left(\omega_{\Lambda}\right)_{i \bar{\jmath}} \delta v^{\Lambda} .
$$

The Kähler moduli $v^{\Lambda}$ also appear in the expression of the volume $\mathcal{V}$ of $Y_{3}$,

$$
\mathcal{V}=\frac{1}{3 !} \int_{Y_{3}} J \wedge J \wedge J=\frac{1}{3 !} \mathcal{V}_{\Lambda \Sigma \Theta} v^{\Lambda} v^{\Sigma} v^{\Theta}
$$


For convenience, we introduce the shorthand notation

$$
\mathcal{V}_{\Lambda}=\frac{1}{2 !} \mathcal{V}_{\Lambda \Sigma \Theta} v^{\Sigma} v^{\Theta}=\partial_{v^{\Lambda}} \mathcal{V}, \quad \mathcal{V}_{\Lambda \Sigma}=\mathcal{V}_{\Lambda \Sigma \Theta} v^{\Theta}=\partial_{v^{\Lambda}} \partial_{v^{\Sigma}} \mathcal{V}
$$

On the other hand, the complex structure moduli space $\mathcal{M}_{\mathrm{cs}}$ is described by suitable complex coordinates $z^{\kappa}$. They are obtained as periods of the holomorphic $(3,0)$-form $\Omega$, and their fluctuations correspond to variations of the components of the Ricci-flat metric with the same kind of indices. More precisely, $\bar{\chi}_{\bar{\kappa}} \in H^{1,2}\left(Y_{3}\right)$ are used to construct $\bar{b}_{\bar{\kappa}} \in H^{0,1}\left(Y_{3} ; T Y_{3}^{1,0}\right)$, where $T Y_{3}^{1,0}$ is the holomorphic tangent bundle to $Y_{3}$, and the $\bar{b}_{\bar{\kappa}}$ encode the metric fluctuations. In our conventions, we have

$$
\delta g_{i j}=\left(\bar{b}_{\bar{\kappa}}\right)_{i j} \delta \bar{z}^{\bar{\kappa}}, \quad\left(\bar{b}_{\bar{\kappa}}\right)_{i}^{\bar{\jmath}}=\frac{i}{\|\Omega\|^{2}}\left(\bar{\chi}_{\bar{\kappa}}\right)_{i \bar{k} \bar{l}} \Omega^{\bar{k} \bar{l} \bar{\jmath}}
$$

Both moduli spaces $\mathcal{M}_{\mathrm{K}}$ and $\mathcal{M}_{\mathrm{cs}}$ are equipped with a natural metric, which can be derived from a potential. These potentials are determined by

$$
e^{\mathcal{K}_{\mathrm{K}}}=\int_{Y_{3}} \frac{1}{3 !} J \wedge J \wedge J, \quad e^{\mathcal{K}_{\mathrm{cs}}}=i \int_{Y_{3}} \Omega \wedge \bar{\Omega},
$$

and yield the metrics

$$
\begin{aligned}
& G_{\Lambda \Sigma}(v)=-\frac{1}{2} \partial_{v^{\Lambda}} \partial_{v^{\Sigma}} \mathcal{K}_{\mathrm{K}}(v)=\frac{1}{2 \mathcal{V}} \int_{Y_{3}} \omega_{\Lambda} \wedge * \omega_{\Sigma}=\frac{1}{2} \frac{\mathcal{V}_{\Lambda} \mathcal{V}_{\Sigma}}{\mathcal{V}^{2}}-\frac{1}{2} \frac{\mathcal{V}_{\Lambda \Sigma}}{\mathcal{V}} \\
& g_{\kappa \bar{\kappa}}(z, \bar{z})=\partial_{z^{\kappa}} \partial_{\bar{z}^{\bar{\kappa}}} \mathcal{K}_{\mathrm{cS}}(z, \bar{z})=-\frac{\int_{Y_{3}} \chi_{\kappa} \wedge \bar{\chi}_{\bar{\kappa}}}{\int_{Y_{3}} \Omega \wedge \bar{\Omega}}
\end{aligned}
$$

\section{E 11d supergravity on a Calabi-Yau threefold}

This appendix is devoted to the presentation of the key points of the Kaluza-Klein reduction of $11 \mathrm{~d}$ supergravity on a Calabi-Yau threefold $Y_{3}$. Zero-modes only are taken into account. For ease of reference we record again the 11d supergravity action

$$
\hat{S}^{(11)}=\int_{\mathcal{M}_{1} 1} \frac{1}{2} \hat{R} \hat{*} 1-\frac{1}{4} \hat{F}_{4} \wedge \hat{*} \hat{F}_{4}-\frac{1}{12} \hat{C}_{3} \wedge \hat{F}_{4} \wedge \hat{F}_{4},
$$

the Kaluza-Klein Ansatz for the three-form

$$
\hat{C}_{3}=\xi^{K} \alpha_{K}-\tilde{\xi}_{K} \beta^{K}+A^{\Lambda} \wedge \omega_{\Lambda}+C_{3},
$$

and the background line element

$$
\left\langle d \hat{s}^{2}\right\rangle=\left\langle\tilde{g}_{\mu \nu}(x)\right\rangle d x^{\mu} d x^{\nu}+2\left\langle g_{i \bar{\jmath}}(y)\right\rangle d y^{i} d \bar{y}^{\bar{\jmath}}
$$

Let us starts discussing the reduction of the Einstein-Hilbert term. The full internal metric $g_{i \bar{\jmath}}$, background and fluctuations, depends on the external coordinates through 
the Kähler moduli $v^{\Lambda}$ and the complex structure moduli $z^{\kappa}$, as can be seen from (D.11), (D.14). Note that the off-diagonal $d x d y$ components of the background metric cannot fluctuate since a Calabi-Yau threefold has no continuous isometries. In order to get a 5d Einstein-Hilbert term with canonical normalization, we have to perform the Weyl rescaling

$$
\tilde{g}_{\mu \nu}=\mathcal{V}^{-2 / 3} g_{\mu \nu}
$$

Straightforward calculation gives then

$$
\int_{\mathcal{M}_{11}} \frac{1}{2} \hat{R} \hat{*} 1=\int_{\mathcal{M}_{5}} \frac{1}{2} R * 1-\frac{1}{2} H_{\Lambda \Sigma}(v) d v^{\Lambda} \wedge * d v^{\Sigma}-g_{\kappa \bar{\kappa}} d z^{\kappa} \wedge * d \bar{z}^{\bar{\kappa}}
$$

where $g_{\kappa \bar{\kappa}}$ is the metric in the complex moduli space, defined in (D.16), and

$$
H_{\Lambda \Sigma}(v)=-G_{\Lambda \Sigma}(v)-\mathcal{V}^{-1} \mathcal{V}_{\Lambda \Sigma}
$$

We have singled out the expression

$$
G_{\Lambda \Sigma}(v)=-\frac{1}{2} \partial_{v^{\Lambda}} \partial_{v^{\Sigma}} \log \mathcal{V}(v)=-\frac{1}{2} \mathcal{V}(v)^{-1} \mathcal{V}_{\Lambda \Sigma \Theta} v^{\Theta}+\frac{1}{8} \mathcal{V}(v)^{-2} \mathcal{V}_{\Lambda \Omega \Theta} \mathcal{V}_{\Sigma \Psi \Xi} v^{\Omega} v^{\Theta} v^{\Psi} v^{\Xi}
$$

because it is the natural metric on the Kähler moduli space. It is useful to define

$$
L^{\Lambda}=\mathcal{V}^{-1 / 3} v^{\Lambda}
$$

since, as we shall see, the kinetic term for $v^{\Lambda}$ expressed in these coordinates takes a particularly simple form. It is crucial to observe that the $L^{\Lambda}$ 's parameterize one degree of freedom less than the $v^{\Lambda}$ 's, since they obey identically $\frac{1}{3 !} \mathcal{V}_{\Lambda \Sigma \Theta} L^{\Lambda} L^{\Sigma} L^{\Theta}=1$. The kinetic term we are interested in reads

$$
-\frac{1}{2} H_{\Lambda \Sigma}(v) d v^{\Lambda} \wedge * d v^{\Sigma}=-\frac{1}{2} G_{\Lambda \Sigma}(L) d L^{\Lambda} \wedge * d L^{\Sigma}-d D \wedge * d D
$$

In this expression, we have defined

$$
D=-\frac{1}{2} \log \mathcal{V}
$$

for future convenience, and we have introduced the symbol $G_{\Lambda \Sigma}(L)$ to denote the metric obtained by replacing $v^{\Lambda}$ by $L^{\Lambda}$ everywhere in (E.7). It is easily checked that $G_{\Lambda \Sigma}(L)$ can be written in a compact form as

$$
G_{\Lambda \Sigma}(L)=\left[-\frac{1}{2} \partial_{L^{\Lambda}} \partial_{L^{\Sigma}} \log \mathcal{N}\right]_{\mathcal{N}=1}=\left[-\frac{1}{2} \mathcal{N}_{\Lambda \Sigma}+\frac{1}{2} \mathcal{N}_{\Lambda} \mathcal{N}_{\Sigma}\right]_{\mathcal{N}=1}
$$

provided that we introduce $\mathcal{N}=\frac{1}{3 !} \mathcal{V}_{\Lambda \Sigma \Theta} L^{\Lambda} L^{\Sigma} L^{\Theta}$.

We are now in a position to describe the reduction of the other terms in the $11 \mathrm{~d}$ Lagrangian. As far as the three-form kinetic term is concerned, a straightforward computation shows that

$$
\begin{aligned}
\int_{\mathcal{M}_{11}}-\frac{1}{4} \hat{F}_{4} \wedge \hat{*} \hat{F}_{4}=\int_{\mathcal{M}_{5}} & +\frac{1}{4}\left(d \tilde{\xi}_{K}-\mathcal{M}_{K M} d \xi^{M}\right)(\operatorname{Im} \mathcal{M})^{-1 K L} \wedge \tilde{*}\left(d \tilde{\xi}_{L}-\mathcal{M}_{L N} d \xi^{N}\right) \\
& -\frac{1}{2} \mathcal{V} G_{\Lambda \Sigma}(v) F^{\Lambda} \wedge \tilde{*} F^{\Sigma}-\frac{1}{4} \mathcal{V} F_{4} \wedge \tilde{*} F_{4}
\end{aligned}
$$


See appendix $\mathrm{D}$ for the definition of $\mathcal{M}$. For the Chern-Simons term, we find

$$
\int_{\mathcal{M}_{11}}-\frac{1}{12} \hat{C}_{3} \wedge \hat{F}_{4} \wedge \hat{F}_{4}=\int_{\mathcal{M}_{5}}-\frac{1}{12} \mathcal{V}_{\Lambda \Sigma \Theta} A^{\Lambda} \wedge F^{\Sigma} \wedge F^{\Theta}+\frac{1}{4}\left(\xi^{K} d \tilde{\xi}_{K}-\tilde{\xi}_{K} d \xi^{K}\right) \wedge F_{4}
$$

As mentioned in the main text, we can dualize the three-form $C_{3}$ into a real scalar $\Phi$. To this end we add to the 5 d action the term

$$
\Delta S^{(5) \mathrm{M}}=\int_{\mathcal{M}_{5}} \frac{1}{4} d \Phi \wedge F_{4}
$$

which implements Bianchi identity $d F_{4}=0$ if we consider $F_{4}$ rather than $C_{3}$ as independent variable. After elimination of $F_{4}$ via its equation of motion, we get

$$
\begin{aligned}
S_{\text {non-grav }}^{(5) \mathrm{M}}=\int_{\mathcal{M}_{5}} & +\frac{1}{4}\left(d \tilde{\xi}_{K}-\mathcal{M}_{K M} d \xi^{M}\right)(\operatorname{Im} \mathcal{M})^{-1 K L} \wedge \tilde{*}\left(d \tilde{\xi}_{L}-\mathcal{M}_{L N} d \xi^{N}\right) \\
& -\frac{1}{2} \mathcal{V} G_{\Lambda \Sigma}(v) F^{\Lambda} \wedge \tilde{*}^{\Sigma}-\frac{1}{12} \mathcal{V}_{\Lambda \Sigma \Theta} A^{\Lambda} \wedge F^{\Sigma} \wedge F^{\Theta} \\
& -\frac{1}{16 \mathcal{V}}\left[\xi^{K} d \tilde{\xi}_{K}-\tilde{\xi}_{K} d \xi^{K}+d \Phi\right] \wedge \tilde{*}\left[\xi^{K} d \tilde{\xi}_{K}-\tilde{\xi}_{K} d \xi^{K}+d \Phi\right] .
\end{aligned}
$$

Let us stress here that we still have to take into account the Weyl rescaling of the metric $\tilde{g}_{\mu \nu}$. It is interesting to note that it is crucial to get the equality between the inverse gauge coupling function and the metric of the moduli space of scalars $L^{\Lambda}$, since

$$
-\frac{1}{2} \mathcal{V} G_{\Lambda \Sigma}(v) F^{\Lambda} \wedge \tilde{*} F^{\Sigma}=-\frac{1}{2} \mathcal{V}^{\frac{2}{3}} G_{\Lambda \Sigma}(v) F^{\Lambda} \wedge * F^{\Sigma}=-\frac{1}{2} G_{\Lambda \Sigma}(L) F^{\Lambda} \wedge * F^{\Sigma}
$$

The final action was given in the main text in (4.14). We only need to specify the quaternionic kinetic for hypermultiplets, which turns out to be

$$
\begin{aligned}
h_{u v} d q^{u} \wedge * d q^{v}= & +d D \wedge * d D+g_{\kappa \bar{\kappa}} d z^{\kappa} \wedge * d \bar{z}^{\bar{\kappa}} \\
& +\frac{1}{4} e^{4 D}\left[d \Phi+\left(\xi^{K} d \tilde{\xi}_{K}-\tilde{\xi}_{K} d \xi^{K}\right)\right]^{2} \\
& -\frac{1}{2} e^{2 D}\left(d \tilde{\xi}_{K}-\mathcal{M}_{K M} d \xi^{M}\right)(\operatorname{Im} \mathcal{M})^{-1 K L}\left(d \tilde{\xi}_{L}-\mathcal{M}_{L N} d \xi^{N}\right) .
\end{aligned}
$$

\section{References}

[1] M. R. Douglas and S. Kachru, "Flux compactification," Rev. Mod. Phys. 79 (2007) 733 [hep-th/0610102];

R. Blumenhagen, B. Kors, D. Lust and S. Stieberger, "Four-dimensional String Compactifications with D-Branes, Orientifolds and Fluxes," Phys. Rept. 445 (2007) 1 [hep-th/0610327].

[2] F. Denef, "Les Houches Lectures on Constructing String Vacua," [arXiv:0803.1194 [hep-th]]. 
[3] W. Taylor, "TASI Lectures on Supergravity and String Vacua in Various Dimensions," [arXiv:1104.2051 [hep-th]].

[4] P. Pasti, D. P. Sorokin and M. Tonin, "On Lorentz invariant actions for chiral p forms," Phys. Rev. D 55 (1997) 6292 [hep-th/9611100].

[5] H. Nishino and E. Sezgin, "New couplings of six-dimensional supergravity," Nucl. Phys. B 505 (1997) 497 [hep-th/9703075].

[6] S. Ferrara, F. Riccioni and A. Sagnotti, "Tensor and vector multiplets in sixdimensional supergravity," Nucl. Phys. B 519 (1998) 115 [hep-th/9711059];

F. Riccioni, A. Sagnotti, "Some properties of tensor multiplets in six-dimensional supergravity," Nucl. Phys. Proc. Suppl. 67 (1998) 68-73. [hep-th/9711077];

F. Riccioni, "Abelian vector multiplets in six-dimensional supergravity," Phys. Lett. B 474 (2000) 79 [hep-th/9910246].

[7] M. Gunaydin, H. Samtleben and E. Sezgin, "On the Magical Supergravities in Six Dimensions," Nucl. Phys. B 848 (2011) 62 [arXiv:1012.1818 [hep-th]].

[8] H. Samtleben, E. Sezgin, R. Wimmer, "(1,0) superconformal models in six dimensions," [arXiv:1108.4060 [hep-th]].

[9] M. R. Douglas, "On D=5 super Yang-Mills theory and $(2,0)$ theory," JHEP 1102, 011 (2011). [arXiv:1012.2880 [hep-th]].

[10] N. Lambert, C. Papageorgakis, M. Schmidt-Sommerfeld, "M5-Branes, D4-Branes and Quantum 5D super-Yang-Mills," JHEP 1101 (2011) 083. [arXiv:1012.2882 [hep-th]].

[11] P. -M. Ho, K. -W. Huang and Y. Matsuo, "A Non-Abelian Self-Dual Gauge Theory in 5+1 Dimensions," JHEP 1107 (2011) 021 [arXiv:1104.4040 [hep-th]].

[12] M. B. Green, J. H. Schwarz, "Anomaly Cancellation in Supersymmetric D=10 Gauge Theory and Superstring Theory," Phys. Lett. B149 (1984) 117-122.

[13] A. Sagnotti, "A Note on the Green-Schwarz mechanism in open string theories," Phys. Lett. B294 (1992) 196-203. [hep-th/9210127].

[14] J. H. Schwarz, "Anomaly - free supersymmetric models in six-dimensions," Phys. Lett. B371 (1996) 223-230. [hep-th/9512053].

[15] C. Vafa, "Evidence for F theory," Nucl. Phys. B469 (1996) 403-418. [hepth/9602022].

[16] D. R. Morrison, C. Vafa, "Compactifications of F theory on Calabi-Yau threefolds. 1," Nucl. Phys. B473 (1996) 74-92. [hep-th/9602114];

D. R. Morrison, C. Vafa, "Compactifications of F theory on Calabi-Yau threefolds. 2.," Nucl. Phys. B476 (1996) 437-469. [hep-th/9603161]. 
[17] S. Ferrara, R. Minasian and A. Sagnotti, "Low-energy analysis of M and F theories on Calabi-Yau threefolds," Nucl. Phys. B 474 (1996) 323 [hep-th/9604097].

[18] V. Sadov, "Generalized Green-Schwarz mechanism in F theory," Phys. Lett. B388 (1996) 45-50. [hep-th/9606008].

[19] V. Kumar and W. Taylor, "String Universality in Six Dimensions," arXiv:0906.0987 [hep-th];

V. Kumar, D. R. Morrison and W. Taylor, "Mapping 6D N = 1 supergravities to F-theory," JHEP 1002 (2010) 099 [arXiv:0911.3393 [hep-th]];

V. Kumar, D. R. Morrison, W. Taylor, "Global aspects of the space of 6D N = 1 supergravities," JHEP 1011 (2010) 118. [arXiv:1008.1062 [hep-th]];

V. Kumar, D. S. Park and W. Taylor, "6D supergravity without tensor multiplets," JHEP 1104 (2011) 080 [arXiv:1011.0726 [hep-th]].

[20] D. S. Park and W. Taylor, "Constraints on 6D Supergravity Theories with Abelian Gauge Symmetry," arXiv:1110.5916 [hep-th].

[21] D. S. Park, "Anomaly Equations and Intersection Theory," arXiv:1111.2351 [hepth].

[22] M. Bershadsky, K. A. Intriligator, S. Kachru, D. R. Morrison, V. Sadov, C. Vafa, "Geometric singularities and enhanced gauge symmetries," Nucl. Phys. B481 (1996) 215-252. [hep-th/9605200].

[23] S. H. Katz and C. Vafa, "Matter from geometry," Nucl. Phys. B 497 (1997) 146 [hep-th/9606086].

[24] D. R. Morrison and W. Taylor, "Matter and singularities," arXiv:1106.3563 [hep-th].

[25] T. W. Grimm, "The N=1 effective action of F-theory compactifications," Nucl. Phys. B 845, 48 (2011) [arXiv:1008.4133 [hep-th]].

[26] T. W. Grimm, H. Hayashi, "F-theory fluxes, Chirality and Chern-Simons theories," [arXiv:1111.1232 [hep-th]].

[27] A. C. Cadavid, A. Ceresole, R. D'Auria, S. Ferrara, "Eleven-dimensional supergravity compactified on Calabi-Yau threefolds," Phys. Lett. B357 (1995) 76-80. [hep-th/9506144].

[28] I. Antoniadis, S. Ferrara, R. Minasian, K. S. Narain, "R**4 couplings in M and type II theories on Calabi-Yau spaces," Nucl. Phys. B507 (1997) 571-588. [hepth/9707013].

[29] T. W. Grimm, W. Taylor, work in progress.

[30] E. Witten, "Phase transitions in M theory and F theory," Nucl. Phys. B471 (1996) 195-216. [hep-th/9603150]. 
[31] K. A. Intriligator, D. R. Morrison, N. Seiberg, "Five-dimensional supersymmetric gauge theories and degenerations of Calabi-Yau spaces," Nucl. Phys. B497 (1997) 56-100.

[32] P. Candelas, E. Perevalov and G. Rajesh, "Toric geometry and enhanced gauge symmetry of F theory / heterotic vacua," Nucl. Phys. B 507 (1997) 445 [hepth/9704097].

[33] S. Ferrara, S. Sabharwal, "Quaternionic Manifolds for Type II Superstring Vacua of Calabi-Yau Spaces," Nucl. Phys. B332 (1990) 317.

[34] L. Andrianopoli, M. Bertolini, A. Ceresole, R. D’Auria, S. Ferrara, P. Fre, T. Magri, "N=2 supergravity and $\mathrm{N}=2$ superYang-Mills theory on general scalar manifolds: Symplectic covariance, gaugings and the momentum map," J. Geom. Phys. 23 (1997) 111-189. [arXiv:hep-th/9605032 [hep-th]].

[35] S. Randjbar-Daemi, A. Salam, E. Sezgin and J. A. Strathdee, "An Anomaly Free Model in Six-Dimensions," Phys. Lett. B 151 (1985) 351.

[36] S. D. Avramis, A. Kehagias and S. Randjbar-Daemi, "A New anomaly-free gauged supergravity in six dimensions," JHEP 0505 (2005) 057 [hep-th/0504033].

[37] S. D. Avramis and A. Kehagias, "A Systematic search for anomaly-free supergravities in six dimensions," JHEP 0510 (2005) 052 [hep-th/0508172].

[38] R. Suzuki and Y. Tachikawa, "More anomaly-free models of six-dimensional gauged supergravity," J. Math. Phys. 47 (2006) 062302 [hep-th/0512019].

[39] A. Ceresole, G. Dall'Agata, "General matter coupled N=2, D = 5 gauged supergravity," Nucl. Phys. B585 (2000) 143-170. [hep-th/0004111].

[40] M. Gunaydin, G. Sierra, P. K. Townsend, "The Geometry of N=2 Maxwell-Einstein Supergravity and Jordan Algebras," Nucl. Phys. B242 (1984) 244.

[41] K. Hanaki, K. Ohashi, Y. Tachikawa, "Supersymmetric Completion of an $\mathrm{R}^{* * 2}$ term in Five-dimensional Supergravity," Prog. Theor. Phys. 117 (2007) 533. [hepth/0611329].

[42] S. Cremonini, K. Hanaki, J. T. Liu, P. Szepietowski, "Black holes in fivedimensional gauged supergravity with higher derivatives," JHEP 0912 (2009) 045. [arXiv:0812.3572 [hep-th]].

[43] E. Cremmer, B. Julia, J. Scherk, "Supergravity Theory in Eleven-Dimensions," Phys. Lett. B76 (1978) 409-412.

[44] T. W. Grimm and R. Savelli, "Gravitational Instantons and Fluxes from M/F-theory on Calabi-Yau fourfolds," arXiv:1109.3191 [hep-th]. 
[45] C. Vafa, E. Witten, "A One loop test of string duality," Nucl. Phys. B447 (1995) 261-270. [hep-th/9505053].

[46] M. J. Duff, J. T. Liu, R. Minasian, "Eleven-dimensional origin of string-string duality: A One loop test," Nucl. Phys. B452 (1995) 261-282. [hep-th/9506126].

[47] M. Haack, J. Louis, "M theory compactified on Calabi-Yau fourfolds with background flux," Phys. Lett. B507 (2001) 296-304. [hep-th/0103068].

[48] R. Friedman, J. Morgan, E. Witten, "Vector bundles and F theory," Commun. Math. Phys. 187 (1997) 679-743. [hep-th/9701162].

[49] A. P. Braun, S. Gerigk, A. Hebecker, H. Triendl, "D7-Brane Moduli vs. FTheory Cycles in Elliptically Fibred Threefolds," Nucl. Phys. B836, 1-36 (2010). [arXiv:0912.1596 [hep-th]].

[50] C. Misner, K.S. Thorne, J.A. Wheeler, Gravitation. San Francisco: W.H. Freeman And Company, 1973.

[51] P. Candelas and X. de la Ossa, "Moduli Space Of Calabi-yau Manifolds," Nucl. Phys. B 355 (1991) 455.

[52] B. R. Greene, "String theory on Calabi-Yau manifolds," hep-th/9702155. 